\title{
Nonlocal multi-scale traffic flow models: analysis beyond vector spaces
}

\author{
Peter E. Kloeden ${ }^{1}$. Thomas Lorenz ${ }^{2}$
}

Received: 22 May 2016 / Accepted: 23 July 2016 / Published online: 11 August 2016 (C) The Author(s) 2016. This article is published with open access at Springerlink.com

\begin{abstract}
Realistic models of traffic flow are nonlinear and involve nonlocal effects in balance laws. Flow characteristics of different types of vehicles, such as cars and trucks, need to be described differently. Two alternatives are used here, $L^{p}$-valued Lebesgue measurable density functions and signed Radon measures. The resulting solution spaces are metric spaces that do not have a linear structure, so the usual convenient methods of functional analysis are no longer applicable. Instead ideas from mutational analysis will be used, in particular the method of Euler compactness will be applied to establish the well-posedness of the nonlocal balance laws. This involves the concatenation of solutions of piecewise linear systems on successive time subintervals obtained by freezing the nonlinear nonlocal coefficients to their values at the start of each subinterval. Various compactness criteria lead to a convergent subsequence. Careful estimates of the linear systems are needed to implement this program.
\end{abstract}

Keywords Nonlocal traffic flow $\cdot$ Balance laws · Well-posedness · Radon measures · Mutational analysis · Euler compactness

Mathematics Subject Classification 35L45 - 35R06 - 35B30 - 34G25 · 49J53

Communicated by Neil Trudinger.

Peter E. Kloeden

kloeden@math.uni-frankfurt.de

Thomas Lorenz

thomas.lorenz@hs-rm.de

1 School of Mathematics and Statistics, Huazhong University of Science and Technology, Wuhan 430074, China

2 Applied Mathematics, RheinMain University of Applied Sciences, Wiesbaden 65197, Germany 


\section{Mathematical models of traffic flow}

A large class of mathematical models of traffic flow is formulated as partial differential equations of hyperbolic type, mostly continuity equations or balance laws. Recently three aspects have been identified which deserve more attention from the analytical point of view.

First, highways and streets usually consist of more than just one lane and, so we need a theory that can handle systems of hyperbolic equations. Second, drivers of vehicles can watch each other and, modern technology (like navigation tools, mobile phones and centrally controlled restrictions on speed) provide facilities to take distant information into consideration. From the mathematical point of view, this leads to nonlocal problems, i.e., the desired solution occurs in the coefficients not just in a pointwise dependence, but in a functional dependence. Convolution operators, for example, are preferred to Nemytskii operators in the quasilinear hyperbolic equations. Nonlocal models of hyperbolic type have already been investigated thoroughly by Colombo, Goatin and collaborators, for example (see [2,16,22-25, 28,36] and references therein).

In addition, recent models try to avoid the a priori assumption that traffic is "sufficiently dense" which is required for describing it in terms of a Lebesgue measurable density. If both vehicles and trucks are using a highway, the traffic control panel has the facilities to specify different restrictions on these groups. Hence it is worth considering them as two components of the traffic system, but the trucks are usually so few that they cannot be described as a Lebesgue measurable density function. From the analytical point of view, the next larger function space (as a generalising alternative) consists of Radon measures. Various models using time-dependent Radon measures have already been published by Carrillo, Colombo, Piccoli and collaborators, for example (see, e.g., [20,21,62-64] and references therein). The main results in [62-64], however, are formulated for the continuity equation or for Radon measures with compact support in $\mathbb{R}^{d}$.

Motivated by the large number of traffic flow models published so far, the focus of our interest is the following class of nonlocal hyperbolic problems

$$
\left\{\begin{array}{l}
\partial_{t} f+\operatorname{div}_{\mathbf{x}}(\widetilde{\mathcal{G}}(t, f, \mu) f)=\widetilde{\mathcal{U}}(t, f, \mu) \cdot f+\widetilde{\mathcal{W}}(t, f, \mu) \\
\partial_{t} \mu+\operatorname{div}_{\mathbf{x}}(\widetilde{\mathcal{B}}(t, f, \mu) \mu)=\widetilde{\mathcal{C}}(t, f, \mu) \cdot \mu
\end{array}\right.
$$

with given initial conditions at time $t=0$. The main goal consists in specifying sufficient conditions on the coefficients for its well-posedness for weakly continuous distributional solutions $f:[0, T] \rightarrow L^{p}\left(\mathbb{R}^{N}\right)$ (with $p>1$ ) and $\mu:[0, T] \rightarrow$ $\mathcal{M}\left(\mathbb{R}^{N}\right)$, where $\mathcal{M}\left(\mathbb{R}^{N}\right)$ denotes the set of finite Radon measures on $\mathbb{R}^{N}$, i.e., to allow their existence, uniqueness as well as the continuous dependence on given data to be established. The coefficient functions here describe the functional dependence, i.e., we regard them as functions

$$
\begin{aligned}
& \widetilde{\mathcal{B}}:[0, T] \times L^{p}\left(\mathbb{R}^{N}\right) \times \mathcal{M}\left(\mathbb{R}^{N}\right) \rightarrow W^{1, \infty}\left(\mathbb{R}^{N}, \mathbb{R}^{N}\right), \\
& \widetilde{\mathcal{C}}:[0, T] \times L^{p}\left(\mathbb{R}^{N}\right) \times \mathcal{M}\left(\mathbb{R}^{N}\right) \rightarrow W^{1, \infty}\left(\mathbb{R}^{N}, \mathbb{R}\right),
\end{aligned}
$$




$$
\begin{aligned}
& \tilde{\mathcal{G}}:[0, T] \times L^{p}\left(\mathbb{R}^{N}\right) \times \mathcal{M}\left(\mathbb{R}^{N}\right) \rightarrow\left\{\mathbf{g} \in L^{\infty}\left(\mathbb{R}^{N}, \mathbb{R}^{N}\right) \cap L^{q} \mid \partial_{\mathbf{y}} \mathbf{g} \in L^{\infty}\right\}, \\
& \tilde{\mathcal{U}}:[0, T] \times L^{p}\left(\mathbb{R}^{N}\right) \times \mathcal{M}\left(\mathbb{R}^{N}\right) \rightarrow\left\{u \in L^{\infty}\left(\mathbb{R}^{N}\right) \cap L^{q}\left(\mathbb{R}^{N}\right) \mid \nabla_{\mathbf{y}} u \in L^{\infty}\right\}, \\
& \widetilde{\mathcal{W}}:[0, T] \times L^{p}\left(\mathbb{R}^{N}\right) \times \mathcal{M}\left(\mathbb{R}^{N}\right) \rightarrow L^{p}\left(\mathbb{R}^{N}\right)
\end{aligned}
$$

because we aim at the most general class of problems for which aspects of nonlocal dependence are characterised by appropriate conditions of boundedness and continuity. From a slightly different perspective, the spatial dependence of the coefficients is completely covered by the function values in $W^{1, \infty}\left(\mathbb{R}^{N}\right), L^{\infty}\left(\mathbb{R}^{N}\right)$ and $L^{p}\left(\mathbb{R}^{N}\right)$, respectively. The pointwise influence of the sought functions $f, \mu$ on their own evolution here is determined by the structure of the respective partial differential equation. It determines essentially to which special examples of traffic flow models the main results can be applied.

In this article we provide new results in various aspects:

- The Lebesgue measurable solution $f$ to the nonlocal transport equation is considered with values in the Banach space $L^{p}\left(\mathbb{R}^{N}\right)$ for some arbitrarily fixed parameter $p \in(1, \infty)$. Most of the publications so far specify weak solutions in $L^{1}$ with bounded total variation in space so the well established theory about hyperbolic balance laws can be applied (see, e.g., [2,23,25,30]). Clearly, the latter function space opens the door for the coefficients to a larger class of pointwise dependence on space, but it restricts the admissible set of solution values significantly. We regard it as realistic in traffic flow models that drivers keep watching their close environment and, this can be modelled in terms of a convolution operator, which need not be symmetric in space and which can have a small support in comparison with the length scale of interest. $L^{p}\left(\mathbb{R}^{N}\right)$ provides the analytical advantage that various types of singularities in spatial densities are covered.

- All coefficients involved can depend on the desired solutions in a functional and, thus, nonlocal way (differently from [20], for example).

- The measure-valued component $\mu$ has its values in the space $\mathcal{M}\left(\mathbb{R}^{N}\right)$ of finite signed Radon measures, i.e., the dual space of continuous functions $\mathbb{R}^{N} \rightarrow \mathbb{R}$ vanishing at infinity supplied with supremum norm (see below for details). Such measure values need not be probability measures or are not assumed to have compact support (as in [62], [63, Section 3], for example).

- The measure-valued component $\mu$ need not be conserved (as, e.g., in [51, Section 2.8], [62]).

The results about well-posedness concern three aspects: Firstly, we specify conditions on the coefficients sufficient for the existence of weak solutions, which do not necessarily imply uniqueness. Similarly to the standard Peano theorem for ordinary differential equations (ODEs), they are based on appropriate continuity and concluded from compactness arguments. Secondly, supplementary assumptions about Lipschitz continuity of the coefficients w.r.t. state imply the uniqueness of weak solutions to the respective initial value problem. Finally, the same conditions of Lipschitz continuity guarantee the continuous dependence of solutions on given data, i.e., initial states and coefficients, which then follows, essentially, from Gronwall's inequality. 


\section{Outline of the paper}

Some basic ideas on mutational analysis are briefly sketched in the next section, Sect. 2. Then, in Sect. 3, the main results on the well-posedness of solutions of the nonlocal balance equations presented in the introduction are stated, first for $L^{p}$-valued solutions and then for Radon measure-valued solutions. The rest of the paper is dedicated to their proofs. Existence results of $L^{p}$-valued solutions of linear transport problems under different regularity assumptions and various estimates needed later are collected in Sect. 4. They are then used in the Euler compactness method in Sect. 5 to provide proofs of the theorems stated in Sect. 3. Finally, Radon measure solutions are handled analogously in Sects. 6 and 7.

\section{Mutational analysis on spaces without a linear structure}

From the analytical point of view, this article provides another novelty in comparison with former publications in this field. It is the underlying concept for constructing solutions. The main idea is to imitate the classical Euler method in combination with compactness arguments. Importantly, this can be realised without special emphasis on the linear structure of the underlying space.

Indeed, whenever the full dynamic problem is too complex for solving it explicitly, one usually relies on some notion of decomposing it into tractable subproblems. Mild solutions to semi-linear evolution equations, for example, combine strongly continuous semi-groups of linear operators with the variation of constants formula leading to fixed-point problems. Galerkin methods are based on the projection on finite-dimensional linear subspaces in combination with the limit for their dimensions tending to infinity and so on. One of the simplest methods is the Euler algorithm, which starts with some discretisation in time and then solves a simpler (usually linear) problem on each time subinterval. Originally introduced for ordinary differential equations (ODEs), this approach has proved to be very powerful in various fields like nonlinear evolution equations or stochastic differential equations (see, e.g., [45,61]). There are many dynamic problems, however, whose states are not in a linear space, but just in a metric space. A large class of examples looks for compact sets in $\mathbb{R}^{N}$ as functions of time which determine their own evolution similarly to ODEs (see, e.g., $[1,29,31,32,46,49,50,57])$.

We focus on the mutational approach to differential equations in metric spaces by Aubin [9-11]. It is similar to, but developed independently from the quasi-differential equations by Panasyuk [58-60], which has been applied by Colombo and collaborators (see, e.g., $[18,26,27]$ and references therein, a comparison in more details is presented in [51,52], for example).

The key idea here is to start with a class of semi-dynamical systems on the (possibly nonlinear) state space. The systems in that class are identified by a parameter. In the standard situation $\mathbb{R}^{N}$, each vector $v \in \mathbb{R}^{N}$ induces an affine semi-dynamical system on $\mathbb{R}^{N}$ defined by $[0, \infty) \times \mathbb{R}^{N} \ni\left(h, \xi_{0}\right) \mapsto \xi_{0}+h \cdot v$. (Here $v \in \mathbb{R}^{N}$ indicates the parameter mentioned before.) A vector $v \in \mathbb{R}^{N}$ is defined as the (right) derivative of a curve $\xi:[0, T) \rightarrow \mathbb{R}^{N}$ at time $t \in[0, T)$ if 


$$
\lim _{h \downarrow 0} \frac{1}{h} \cdot|\xi(t+h)-(\xi(t)+h \cdot v)|=0 .
$$

For an ODE $x^{\prime}=f(t, x)$, a vector field function $f:[0, T] \times \mathbb{R}^{N} \rightarrow \mathbb{R}^{N}$ is given which prescribes the right derivative of the wanted curve in dependence of time $t$ and respective state.

In the (not necessarily linear, but) metric setting $(X, d)$, a family of semi-dynamical systems $\vartheta_{p}:[0, \infty) \times X \rightarrow X$ for $p \in \mathbb{P}$ plays the role of the tangent vectors $v \in \mathbb{R}^{N}$ and their corresponding affine maps. For a curve $x:[0, T] \rightarrow X, \vartheta_{p}$ represents the counterpart of the right derivative at time $t \in[0, T)$ if it induces a first-order approximation in the sense that

$$
\lim _{h \downarrow 0} \frac{1}{h} \cdot d\left(x(t+h), \vartheta_{p}(h, x(t))\right)=0 .
$$

This notion does not rely on any aspects of linear structure of $X$, but merely on distances. The final step for generalising ODEs is now to prescribe the parameter $p \in \mathbb{P}$ as a function of time $t \in[0, T)$ and state in $X$. This leads to a so-called mutational equation on the tuple $\left(X, d,\left(\vartheta_{p}\right)_{p \in \mathbb{P}}\right)$.

They were introduced by Jean-Pierre Aubin as a joint environment for evolving vectors in $\mathbb{R}^{N}$ and compact subsets of $\mathbb{R}^{N}$ [9-11] and then extended by the authors for various further examples like stochastic differential equations with nonlocal sample dependence, random closed sets in a Hilbert space and fuzzy differential equations with evolving membership grade (see, e.g., [41-44,51,52]).

A key goal in mutational analysis so far has been to specify conditions on the given semi-dynamical systems $\vartheta_{p}, p \in \mathbb{P}$, sufficient for the well-posedness of initial value problems if the given feedback function satisfies standard assumptions like continuity and Lipschitz continuity, respectively. The main approach for constructing solution is always the Euler algorithm, so there is a special interest in the "error propagation" along two Euler approximations for later conclusions about their maximal distance while the time step is tending to 0 . This basic notion has the immediate consequence that any two components (or more) fitting in this framework can be combined in a system immediately, i.e., sufficient conditions for the well-posedness of systems are available without any additional considerations. That is the essential aspect motivating our interest in this mutational framework. In this article, particularly, we are free to consider the solutions with values in $L^{p}\left(\mathbb{R}^{N}\right)$ and $\mathcal{M}\left(\mathbb{R}^{N}\right)$ separately.

Finally, our main goal here is not to advertise some further abstract theory providing yet another generalised solution to differential equations in metric spaces. Instead, we want to emphasise the benefit of mutational analysis of specifying explicit continuity conditions on the semi-dynamical systems which guarantee the convergence of the Euler method, in a generalised sense, however. This makes it significantly easier to check whether a decomposition of the full dynamic problem into a class of simpler problems (inducing the semi-dynamical systems) plus feedback will succeed or not. Then, in the very end and for each example individually, it is recommended to identify an established solution criterion which the existing curve in the metric space satisfies. In the concrete examples of nonlocal balance laws here, the curves are $L^{p}$ bounded, 
weakly continuous weak solutions and narrowly continuous distributional solutions with values in the space of signed Radon measures, respectively. These connections to partial differential equations specify essential differences of our results from recent contributions of Aubin et al. to traffic flow modelling (see, e.g., [12-14]).

\section{The main results}

The main results on the existence and uniqueness of various kinds of solutions to the nonlocal balance equations are stated in this section and will be proved later after developments of other background results. These results are stated in terms of the following metrics and convergence.

Definition 1 For $p \in(1, \infty)$ fixed and $q:=\frac{p}{p-1}$, define $d_{L^{p}}: L^{p}\left(\mathbb{R}^{N}\right) \times L^{p}\left(\mathbb{R}^{N}\right) \rightarrow$ $[0, \infty)$

$$
\begin{aligned}
& d_{L^{p}}(f, g):=\sup \left\{\int_{\mathbb{R}^{N}} \varphi \cdot(f-g) d \mathbf{x} \mid \varphi \in C_{c}^{1}\left(\mathbb{R}^{N}\right),\|\varphi\|_{L^{q}} \leq 1,\|\varphi\|_{L^{\infty}} \leq 1,\right. \\
&\left.\left\|\nabla_{\mathbf{x}} \varphi\right\|_{L^{\infty}} \leq 1\right\} .
\end{aligned}
$$

Here $C_{c}^{0}\left(\mathbb{R}^{N}\right)$ denotes the space of continuous functions $\mathbb{R}^{N} \rightarrow \mathbb{R}$ with compact support and $C_{0}^{0}\left(\mathbb{R}^{N}\right)$ its closure with respect to the supremum norm, respectively. Furthermore, $\mathcal{M}\left(\mathbb{R}^{N}\right)$ consists of all finite real-valued Radon measures on $\mathbb{R}^{N}$, i.e., it is the dual space of $\left(C_{0}^{0}\left(\mathbb{R}^{N}\right),\|\cdot\|_{L^{\infty}}\right)$ (due to the Riesz theorem [7, Remark 1.57]), and $\mathcal{M}^{+}\left(\mathbb{R}^{N}\right)$ denotes the subset of nonnegative measures $\mu \in \mathcal{M}\left(\mathbb{R}^{N}\right)$, i.e. $\mu(\cdot) \geq 0$.

Definition 2 A sequence $\left(\mu_{n}\right)_{n \in \mathbb{N}}$ in $\mathcal{M}\left(\mathbb{R}^{N}\right)$ is said to converge narrowly to $\mu \in$ $\mathcal{M}\left(\mathbb{R}^{N}\right)$ if for every bounded continuous function $\varphi: \mathbb{R}^{N} \rightarrow \mathbb{R}$,

$$
\lim _{n \rightarrow \infty} \int_{\mathbb{R}^{N}} \varphi d \mu_{n}=\int_{\mathbb{R}^{N}} \varphi d \mu
$$

Definition 3 ([51, Definition 2.42], [15, Section 8.3]) The mapping $\mathcal{M}\left(\mathbb{R}^{N}\right) \times$ $\mathcal{M}\left(\mathbb{R}^{N}\right) \rightarrow[0, \infty)$ defined by

$$
(\mu, v) \mapsto \sup \left\{\int_{\mathbb{R}^{N}} \psi d(\mu-v) \mid \psi \in C^{1}\left(\mathbb{R}^{N}\right),\|\psi\|_{L^{\infty}},\|\nabla \psi\|_{L^{\infty}} \leq 1\right\}
$$

is called the $W^{1, \infty}$ dual metric $d_{\mathcal{M}}$ or the Kantorovich-Rubinstein metric on $\mathcal{M}\left(\mathbb{R}^{N}\right)$.

\section{1 $L^{p}\left(\mathbb{R}^{N}\right)$-valued solutions to nonlocal balance laws}

Proofs of the following theorem and propositions are given in Sect. 5. 
Theorem 4 (Existence of solutions in $L^{p}\left(\mathbb{R}^{N}\right)$ ) Consider the initial value problem

$$
\partial_{t} f+\operatorname{div}_{\mathbf{x}}(\mathcal{G}(t, f) f)=\mathcal{U}(t, f) \cdot f+\mathcal{W}(t, f), \quad f(0)=f_{0}
$$

with the coefficient functions

$$
\begin{aligned}
& \mathcal{G}:[0, T] \times L^{p}\left(\mathbb{R}^{N}\right) \rightarrow\left\{\mathbf{g} \in L^{\infty}\left(\mathbb{R}^{N}, \mathbb{R}^{N}\right) \cap L^{q} \mid \partial_{\mathbf{y}} \mathbf{g} \in L^{\infty}\right\}, \\
& \mathcal{U}:[0, T] \times L^{p}\left(\mathbb{R}^{N}\right) \rightarrow\left\{u \in L^{\infty}\left(\mathbb{R}^{N}\right) \cap L^{q}\left(\mathbb{R}^{N}\right) \mid \nabla_{\mathbf{y}} u \in L^{\infty}\right\}, \\
& \mathcal{W}:[0, T] \times L^{p}\left(\mathbb{R}^{N}\right) \rightarrow L^{p}\left(\mathbb{R}^{N}\right)
\end{aligned}
$$

under the following assumptions:

(i) (Global a priori bounds) $\sup _{t, f}\left(\left\|\operatorname{div}_{\mathbf{x}} \mathcal{G}(t, f)\right\|_{L^{\infty}\left(\mathbb{R}^{N}\right)}+\|\mathcal{U}(t, f)\|_{L^{\infty}\left(\mathbb{R}^{N}\right)}\right.$ $\left.+\|\mathcal{W}(t, f)\|_{L^{p}\left(\mathbb{R}^{N}\right)}\right)<\infty$

(ii) (Locally uniform a priori bounds) For every $r>0$, there exists a constant $C_{r}<\infty$ such that for all $t \in[0, T]$ and $f \in L^{p}\left(\mathbb{R}^{N}\right)$ with $\|f\|_{L^{p}\left(\mathbb{R}^{N}\right)} \leq r$,

$$
\begin{aligned}
& \|\mathcal{G}(t, f)\|_{L^{\infty}\left(\mathbb{R}^{N}\right)} \\
& \quad+\left\|\partial_{\mathbf{x}} \mathcal{G}(t, f)\right\|_{L^{\infty}\left(\mathbb{R}^{N}, \mathbb{R}^{N \times N}\right)} \leq C_{r}, \quad\left\|\nabla_{\mathbf{x}} \mathcal{U}(t, f)\right\|_{L^{\infty}\left(\mathbb{R}^{N}, \mathbb{R}^{N}\right)} \leq C_{r} .
\end{aligned}
$$

(iii) (Locally uniform choice of function dominating values of $\mathcal{W}$ ) For every radius $r>0$, there exist $\widehat{w} \in L^{p}\left(\mathbb{R}^{N}\right)$ and a compact set $K_{\mathbf{X}} \subset \mathbb{R}^{N}$ such that for all $t \in[0, T]$ and $f \in L^{p}\left(\mathbb{R}^{N}\right)$ with $\|f\|_{L^{p}\left(\mathbb{R}^{N}\right)} \leq r$,

$$
|\mathcal{W}(t, f)(\cdot)| \leq \widehat{w}(\cdot) \quad \text { Lebesgue-almost everywhere in } \mathbb{R}^{N} \backslash K_{\mathbf{x}}
$$

(iv) $\mathcal{G}, \mathcal{U}:[0, T] \times\left(L^{p}\left(\mathbb{R}^{N}\right), d_{L^{p}}\right) \rightarrow\left(L^{q},\|\cdot\|_{L^{q}}\right)$ and $\mathcal{W}:[0, T] \times$ $\left(L^{p}\left(\mathbb{R}^{N}\right), d_{L^{p}}\right) \rightarrow\left(L^{p},\|\cdot\|_{L^{p}}\right)$ are measurable.

(v) (Continuity) The functions $\mathcal{G}(t, \cdot), \mathcal{U}(t, \cdot):\left(L^{p}\left(\mathbb{R}^{N}\right), d_{L^{p}}\right) \rightarrow\left(L^{q},\|\cdot\|_{L^{q}}\right)$ and $\mathcal{W}(t, \cdot):\left(L^{p}\left(\mathbb{R}^{N}\right), d_{L^{p}}\right) \rightarrow\left(L^{p},\|\cdot\|_{L^{p}}\right)$ are continuous for Lebesgue-almost every $t \in[0, T]$ :

Then every initial value problem related to (1) has a weak solution $f:[0, T] \rightarrow$ $L^{p}\left(\mathbb{R}^{N}\right)$ in the following sense: $f:[0, T] \rightarrow\left(L^{p}\left(\mathbb{R}^{N}\right)\right.$, weak $)$ is continuous with $f(0)=f_{0}$ and

$$
\begin{aligned}
\int_{\mathbb{R}^{N}} \varphi\left(f\left(t_{2}\right)-f\left(t_{1}\right)\right) d \mathbf{x}= & \int_{t_{1}}^{t_{2}} \int_{\mathbb{R}^{N}} f(s, \mathbf{x}) \mathcal{G}(s, f(s))(\mathbf{x}) \cdot \nabla_{\mathbf{x}} \varphi(\mathbf{x}) d \mathbf{x} d s \\
& +\int_{t_{1}}^{t_{2}} \int_{\mathbb{R}^{N}}(f(s, \mathbf{x}) \mathcal{U}(s, f(s))(\mathbf{x}) \\
& +\mathcal{W}(s, f(s))(\mathbf{x})) \varphi(\mathbf{x}) d \mathbf{x} d s
\end{aligned}
$$


for any $0 \leq t_{1}<t_{2} \leq T, \varphi \in C_{c}^{1}\left(\mathbb{R}^{N}\right)$. Furthermore this function $f(\cdot)$ is bounded w.r.t. the $L^{p}\left(\mathbb{R}^{N}\right)$ norm and continuous w.r.t. $d_{L^{p}}$.

In hypothesis (i), the global a priori bound on $\|\mathcal{W}(t, f)\|_{L^{p}\left(\mathbb{R}^{N}\right)}$ can be weakened to a linear growth condition w.r.t. $\|f\|_{L^{p}\left(\mathbb{R}^{N}\right)}$ since the subsequent considerations and the Gronwall's inequality still imply an a priori estimate of the $L^{p}\left(\mathbb{R}^{N}\right)$ norm of weak solutions at each time instant.

Proposition 5 (Uniqueness of weak solutions in $L^{p}\left(\mathbb{R}^{N}\right)$ ) Consider the initial value problem (1) with the coefficient functions

$$
\begin{aligned}
& \mathcal{G}:[0, T] \times L^{p}\left(\mathbb{R}^{N}\right) \rightarrow\left\{\mathbf{g} \in L^{\infty}\left(\mathbb{R}^{N}, \mathbb{R}^{N}\right) \cap L^{q} \mid \partial_{\mathbf{y}} \mathbf{g} \in L^{\infty}\right\}, \\
& \mathcal{U}:[0, T] \times L^{p}\left(\mathbb{R}^{N}\right) \rightarrow\left\{u \in L^{\infty}\left(\mathbb{R}^{N}\right) \cap L^{q}\left(\mathbb{R}^{N}\right) \mid \nabla_{\mathbf{y}} u \in L^{\infty}\right\}, \\
& \mathcal{W}:[0, T] \times L^{p}\left(\mathbb{R}^{N}\right) \rightarrow L^{p}\left(\mathbb{R}^{N}\right)
\end{aligned}
$$

under the assumptions (i)-(iv) of Theorem 4 and the hypothesis

(vi') (Locally uniform Lipschitz conditions w.r.t. states) For every $r>0$, there exists a constant $\Lambda_{r}>0$ such that

$$
\begin{aligned}
\left\|\mathcal{G}\left(t, f_{1}\right)-\mathcal{G}\left(t, f_{2}\right)\right\|_{L^{q}\left(\mathbb{R}^{N}, \mathbb{R}^{N}\right)} & \leq \Lambda_{r} \cdot d_{L^{p}}\left(f_{1}, f_{2}\right), \\
\left\|\mathcal{U}\left(t, f_{1}\right)-\mathcal{U}\left(t, f_{2}\right)\right\|_{L^{q}\left(\mathbb{R}^{N}\right)} & \leq \Lambda_{r} \cdot d_{L^{p}}\left(f_{1}, f_{2}\right), \\
\left\|\mathcal{W}\left(t, f_{1}\right)-\mathcal{W}\left(t, f_{2}\right)\right\|_{L^{p}\left(\mathbb{R}^{N}\right)} & \leq \Lambda_{r} \cdot d_{L^{p}}\left(f_{1}, f_{2}\right)
\end{aligned}
$$

holds for all $t \in[0, T]$ and $f_{1}, f_{2} \in L^{p}\left(\mathbb{R}^{N}\right)$ with $\left\|f_{1}\right\|_{L^{p}\left(\mathbb{R}^{N}\right)},\left\|f_{2}\right\|_{L^{p}\left(\mathbb{R}^{N}\right)} \leq r$.

Then for each initial state $f_{0} \in L^{p}\left(\mathbb{R}^{N}\right)$, the continuous weak solution $f$ : $[0, T] \rightarrow\left(L^{p}\left(\mathbb{R}^{N}\right)\right.$, weak $)$ to the nonlinear problem (1) satisfying the measurability conditions associated with

$$
\mathcal{G}(\cdot, f), \partial_{\mathbf{x}} \mathcal{G}(\cdot, f), \mathcal{U}(\cdot, f) \in L^{\infty}\left(0, T ; L^{\infty}\right), \quad \mathcal{W}(\cdot, f) \in L^{1}\left(0, T ; L^{p}\right)
$$

is unique.

Proposition 6 (Lipschitz continuous dependence on given data) Let $\mathcal{G}^{(j)}, \mathcal{U}^{(j)}$ and $\mathcal{W}^{(j)}$ satisfy the assumptions of Proposition 5 (with the same a priori bounds). In addition, let $f^{(j)}$ denote the unique weak solution of problem (1) for the coefficients $\mathcal{G}^{(j)}, \mathcal{U}^{(j)}, \mathcal{W}^{(j)}$ and the initial state $f_{0}^{(j)} \in L^{p}\left(\mathbb{R}^{N}\right), j=1,2$, respectively. Choose some $R>0$ with $\max _{j=1,2}\left\|f_{0}^{(j)}\right\|_{L^{p}\left(\mathbb{R}^{N}\right)} \leq R$ and set

$$
\begin{aligned}
\Delta(t, C):= & \sup _{\substack{\zeta \in L^{p}\left(\mathbb{R}^{N}\right): \\
\|\zeta\|_{L^{p}} \leq C}}\left\|\mathcal{G}^{(1)}(t, \zeta)-\mathcal{G}^{(2)}(t, \zeta)\right\|_{L^{q}\left(\mathbb{R}^{N}, \mathbb{R}^{N}\right)} \\
& +\sup _{\substack{\zeta \in L^{p}\left(\mathbb{R}^{N}\right): \\
\|\zeta\|_{L^{p}} \leq C}}\left\|\mathcal{U}^{(1)}(t, \zeta)-\mathcal{U}^{(2)}(t, \zeta)\right\|_{L^{q}\left(\mathbb{R}^{N}\right)}
\end{aligned}
$$




$$
+\sup _{\substack{\zeta \in L^{p}\left(\mathbb{R}^{N}\right): \\\|\zeta\|_{L^{p} \leq C}}}\left\|\mathcal{W}^{(1)}(t, \zeta)-\mathcal{W}^{(2)}(t, \zeta)\right\|_{L^{p}\left(\mathbb{R}^{N}\right)}
$$

Then there exists a constant $C>0$ depending on $R, T$ and the constants of coefficient functions (in assumptions (i)-(iv),(vi')) such that for every $t \in[0, T]$,

$$
d_{L^{p}}\left(f^{(1)}(t), f^{(2)}(t)\right) \leq e^{C t} \cdot\left(d_{L^{p}}\left(f_{0}^{(1)}, f_{0}^{(2)}\right)+C \cdot \int_{0}^{t} \Delta(s, C) d s\right) .
$$

\subsection{Measure-valued solutions to nonlocal balance laws}

Proofs of the following theorems and propositions are given in Sect. 7.

Theorem 7 (Existence of distributional solutions with values in $\mathcal{M}\left(\mathbb{R}^{N}\right)$ ) Consider the initial value problem

$$
\partial_{t} \mu+\operatorname{div}_{\mathbf{x}}(\mathcal{B}(t, \mu) \mu)=\mathcal{C}(t, \mu) \cdot \mu \quad \text { in }[0, T], \quad \mu(0)=\mu_{0}
$$

with the coefficient functions $\mathcal{B}:[0, T] \times \mathcal{M}\left(\mathbb{R}^{N}\right) \rightarrow W^{1, \infty}\left(\mathbb{R}^{N}, \mathbb{R}^{N}\right), \mathcal{C}:[0, T] \times$ $\mathcal{M}\left(\mathbb{R}^{N}\right) \rightarrow W^{1, \infty}\left(\mathbb{R}^{N}, \mathbb{R}\right)$ under the following assumptions:

(i) (Global a priori bounds) $\sup _{t, \zeta}\left(\|\mathcal{B}(t, \zeta)\|_{L^{\infty}\left(\mathbb{R}^{N}, \mathbb{R}^{N}\right)}+\|\mathcal{C}(t, \zeta)\|_{L^{\infty}\left(\mathbb{R}^{N}\right)}\right)<\infty$

(ii) (Locally uniform a priori bounds) For every $r>0$, there exists a constant $C_{r}<\infty$ such that

$$
\left\|\partial_{\mathbf{x}} \mathcal{B}(t, \zeta)\right\|_{L^{\infty}\left(\mathbb{R}^{N}, \mathbb{R}^{N \times N}\right)} \leq C_{r}, \quad\left\|\nabla_{\mathbf{x}} \mathcal{C}(t, \zeta)\right\|_{L^{\infty}\left(\mathbb{R}^{N}, \mathbb{R}^{N}\right)} \leq C_{r}
$$

holds for all $t \in[0, T], \zeta \in \mathcal{M}\left(\mathbb{R}^{N}\right)$ with $|\zeta|\left(\mathbb{R}^{N}\right) \leq r$.

(iii) The functions $\mathcal{B}(\cdot, \zeta), \mathcal{C}(\cdot, \zeta):[0, T] \rightarrow\left(L^{\infty},\|\cdot\|_{L^{\infty}}\right)$ are measurable for each $\zeta \in \mathcal{M}\left(\mathbb{R}^{N}\right)$.

(iv) (Continuity w.r.t. states) The functions $\mathcal{B}(t, \cdot), \mathcal{C}(t, \cdot):\left(\mathcal{M}\left(\mathbb{R}^{N}\right), d_{\mathcal{M}}\right) \rightarrow$ $\left(L^{\infty},\|\cdot\|_{L^{\infty}}\right)$ are continuous for Lebesgue-almost every $t \in[0, T]$.

Then every initial value problem related to (3) has a narrowly continuous distributional solution $\mu:[0, T] \rightarrow \mathcal{M}\left(\mathbb{R}^{N}\right)$ in the sense that $\mu:[0, T] \rightarrow$ $\left(\mathcal{M}\left(\mathbb{R}^{N}\right)\right.$, narrow $), t \mapsto \mu_{t}$ is continuous for $\mu_{0}$ given and

$$
\begin{aligned}
\int_{\mathbb{R}^{N}} \varphi d \mu_{t_{2}}-\int_{\mathbb{R}^{N}} \varphi d \mu_{t_{1}}= & \int_{t_{1}}^{t_{2}} \int_{\mathbb{R}^{N}} \mathcal{B}\left(s, \mu_{s}\right)(\mathbf{x}) \cdot \nabla_{\mathbf{x}} \varphi(\mathbf{x}) d \mu_{s}(\mathbf{x}) d s \\
& +\int_{t_{1}}^{t_{2}} \int_{\mathbb{R}^{N}} \mathcal{C}\left(s, \mu_{s}\right)(\mathbf{x}) \varphi(\mathbf{x}) d \mu_{s}(\mathbf{x}) d s
\end{aligned}
$$

for any $0 \leq t_{1}<t_{2} \leq T, \varphi \in C_{c}^{1}\left(\mathbb{R}^{N}\right)$. Furthermore, this function $\mu(\cdot)$ is bounded w.r.t. the total variation, has tight values and is continuous w.r.t. $d_{\mathcal{M}}$. 
Proposition 8 (Uniqueness of distributional measure-valued solutions) Consider the same initial value problem (3) with the coefficient functions

$\mathcal{B}:[0, T] \times \mathcal{M}\left(\mathbb{R}^{N}\right) \rightarrow W^{1, \infty}\left(\mathbb{R}^{N}, \mathbb{R}^{N}\right), \quad \mathcal{C}:[0, T] \times \mathcal{M}\left(\mathbb{R}^{N}\right) \rightarrow W^{1, \infty}\left(\mathbb{R}^{N}, \mathbb{R}\right)$

under the assumptions (i)-(iii) of Theorem 7 and the hypothesis

$\left(v^{\prime}\right)$ (Locally uniform Lipschitz conditions w.r.t. states) For every $r>0$ there exists a constant $\Lambda_{r}>0$ such that

$$
\begin{aligned}
\left\|\mathcal{B}\left(t, \zeta_{1}\right)-\mathcal{B}\left(t, \zeta_{2}\right)\right\|_{L^{\infty}\left(\mathbb{R}^{N}, \mathbb{R}^{N}\right)} & \leq \Lambda_{r} \cdot d_{\mathcal{M}}\left(\zeta_{1}, \zeta_{2}\right) \\
\left\|\mathcal{C}\left(t, \zeta_{1}\right)-\mathcal{C}\left(t, \zeta_{2}\right)\right\|_{L^{\infty}\left(\mathbb{R}^{N}\right)} & \leq \Lambda_{r} \cdot d_{\mathcal{M}}\left(\zeta_{1}, \zeta_{2}\right)
\end{aligned}
$$

for all $t \in[0, T]$ and $\zeta_{1}, \zeta_{2} \in \mathcal{M}\left(\mathbb{R}^{N}\right)$ with $\left|\zeta_{1}\right|\left(\mathbb{R}^{N}\right),\left|\zeta_{2}\right|\left(\mathbb{R}^{N}\right) \leq r$.

Then for each initial Radon measure $\mu_{0} \in \mathcal{M}\left(\mathbb{R}^{N}\right)$, the narrowly continuous distributional solution $\mu:[0, T] \rightarrow \mathcal{M}\left(\mathbb{R}^{N}\right)$ to nonlocal initial value problem (3) is unique.

Proposition 9 (Lipschitz continuous dependence on given data) Let $\mathcal{B}^{(j)}$ and $\mathcal{C}^{(j)}$ satisfy the assumptions of Proposition 8 (with the same a priori bounds). In addition, let $\mu^{(j)}$ denote the unique distributional solution of problem (3) related to the coefficients $\mathcal{B}^{(j)}, \mathcal{C}^{(j)}$ and the initial measure $\mu_{0}^{(j)} \in \mathcal{M}\left(\mathbb{R}^{N}\right), j=1,2$, respectively. Choose some $R>0$ with $\max _{j=1,2}\left|\mu_{0}^{(j)}\right|\left(\mathbb{R}^{N}\right) \leq R$ and set

$$
\begin{aligned}
\Delta(t, C):= & \sup _{\substack{\zeta \in \mathcal{M}\left(\mathbb{R}^{N}\right): \\
|\zeta|\left(\mathbb{R}^{N}\right) \leq C}}\left\|\mathcal{B}^{(1)}(t, \zeta)-\mathcal{B}^{(2)}(t, \zeta)\right\|_{L^{\infty}} \\
& +\sup _{\substack{\zeta \in \mathcal{M}\left(\mathbb{R}^{N}\right): \\
|\zeta|\left(\mathbb{R}^{N}\right) \leq C}}\left\|\mathcal{C}^{(1)}(t, \zeta)-\mathcal{C}^{(2)}(t, \zeta)\right\|_{L^{\infty}}
\end{aligned}
$$

Then there exists a constant $C>0$ depending on $R, T$ and the constants of coefficient functions (in assumptions (i)-(iii) and $\left(v^{\prime}\right)$ ) such that

$$
d_{\mathcal{M}}\left(\mu_{t}^{(1)}, \mu_{t}^{(2)}\right) \leq e^{C t} \cdot\left(d_{\mathcal{M}}\left(\mu_{0}^{(1)}, \mu_{0}^{(2)}\right)+C \cdot \int_{0}^{t} \Delta(s, C) d s\right)
$$

for every $t \in[0, T]$.

\section{$4 L^{p}\left(\mathbb{R}^{N}\right)$-valued solutions to linear balance laws}

\subsection{The metrics $d_{L^{p}}, e_{L^{p}}$ of the state space $L^{p}\left(\mathbb{R}^{N}\right)$}

In Definition 1 , we have already specified the metric $d_{L^{p}}$ on $L^{p}\left(\mathbb{R}^{N}\right)$. It will mainly serve the comparison of two densities at the same time instant. Now two further metrics 
on $L^{p}\left(\mathbb{R}^{N}\right)$ follow. They will be used for describing the regularity of weak solutions with respect to time.

Definition 10 Let $1<p<\infty$ and $q>1$ with $\frac{1}{p}+\frac{1}{q}=1$ and define $\breve{e}_{L^{p}}, e_{L^{p}}$ : $L^{p}\left(\mathbb{R}^{N}\right) \times L^{p}\left(\mathbb{R}^{N}\right) \rightarrow[0, \infty)$ as

$\breve{e}_{L^{p}}(f, g):=\sup \left\{\int_{\mathbb{R}^{N}} \varphi \cdot(f-g) d \mathbf{x} \mid \varphi \in C_{c}^{1}\left(\mathbb{R}^{N}\right),\|\varphi\|_{W^{1, q}} \leq 1,\|\varphi\|_{W^{1, \infty}} \leq 1\right\}$

$e_{L^{p}}(f, g):=\breve{e}_{L^{p}}(f, g)+\left|\|f\|_{L^{p}\left(\mathbb{R}^{N}\right)}-\|g\|_{L^{p}\left(\mathbb{R}^{N}\right)}\right|$.

Remark 11 Obviously the following inequalities hold for all $f, g \in L^{p}\left(\mathbb{R}^{N}\right)$ :

$$
\breve{e}_{L^{p}}(f, g) \leq d_{L^{p}}(f, g) \leq\|f-g\|_{L^{p}\left(\mathbb{R}^{N}\right)}, \quad e_{L^{p}}(f, g) \leq 2 \cdot\|f-g\|_{L^{p}\left(\mathbb{R}^{N}\right)} .
$$

Remark 12 The metrics $d_{L^{p}}$ and $\breve{e}_{L^{p}}$ are constructed in a very similar way, namely in terms of a supremum for all test functions in a unit ball. What differs, however, is the class of test functions and the norm underlying the unit ball. From a more general point of view, they both modify the $L^{p}$ norm. Indeed, the well-known Hahn-Banach theorem implies for every $f, g \in L^{p}\left(\mathbb{R}^{N}\right)$

$$
\begin{aligned}
\|f-g\|_{L^{p}\left(\mathbb{R}^{N}\right)} & =\sup \left\{\int_{\mathbb{R}^{N}} \varphi \cdot(f-g) d \mathbf{x} \mid \varphi \in L^{q}\left(\mathbb{R}^{N}\right),\|\varphi\|_{L^{q}} \leq 1\right\} \\
& =\sup \left\{\int_{\mathbb{R}^{N}} \varphi \cdot(f-g) d \mathbf{x} \mid \varphi \in C_{c}^{1}\left(\mathbb{R}^{N}\right),\|\varphi\|_{L^{q}} \leq 1\right\}
\end{aligned}
$$

since $L^{q}\left(\mathbb{R}^{N}\right)$ represents the dual space of $L^{p}\left(\mathbb{R}^{N}\right)$ and $C_{c}^{1}\left(\mathbb{R}^{N}\right)$ is dense in the Banach space $L^{q}\left(\mathbb{R}^{N}\right)$. The main difference concerns the class of test functions. For the metric $d_{L^{p}}$ (concerning distances between density functions at the same time instant later on), for example, we additionally suppose every test function $\varphi \in C_{c}^{1}\left(\mathbb{R}^{N}\right)$ to be in the unit ball of $W^{1, \infty}\left(\mathbb{R}^{N}\right)$, i.e., $\varphi$ is bounded and 1-Lipschitz continuous in addition. Hence, the related functional

$$
L^{p}\left(\mathbb{R}^{N}\right) \longrightarrow \mathbb{R}, \quad f \longmapsto \int_{\mathbb{R}^{N}} \varphi \cdot f d \mathbf{x}
$$

might hardly change when comparing two density functions $f, g \in L^{p}\left(\mathbb{R}^{N}\right)$ although the $L^{p}$ norm $\|f-g\|_{L^{p}\left(\mathbb{R}^{N}\right)}$ is rather large. Similar modifications of the norm have already proved to be useful for $L^{2}$ density functions in cancer cell migration models in $[53,54]$, for example.

In this subsection we specify relations between the metrics $d_{L^{p}}, \breve{e}_{L^{p}}, e_{L^{p}}$ and more established topologies like the $L^{p}\left(\mathbb{R}^{N}\right)$ norm and the weak topology. Then in Sect. 6.1 below, the notion is adapted to signed Radon measures on $\mathbb{R}^{N}$ (see Remark 41 for more details). 
Lemma $13 \breve{e}_{L^{p}}$ metrizes the weak topology on norm-bounded tight balls in $L^{p}\left(\mathbb{R}^{N}\right)$ in the following sense: Suppose $f \in L^{p}\left(\mathbb{R}^{N}\right)$ and let $\left(f_{k}\right)_{k \in \mathbb{N}}$ be any sequence in $L^{p}\left(\mathbb{R}^{N}\right)$ such that $\left(\left|f_{k}\right|^{p}\right)$ is tight in $\mathbb{R}^{N}$, i.e.,

$$
\lim _{\rho \rightarrow \infty} \sup _{k \in \mathbb{N}}\left\|f_{k}\right\|_{L^{p}\left(\mathbb{R}^{N} \backslash \mathbb{B}_{\rho}(0)\right)}=0 .
$$

Then,

$$
f_{k} \rightarrow \text { fweakly in } L^{p}\left(\mathbb{R}^{N}\right)(k \rightarrow \infty) \Leftrightarrow\left\{\begin{array}{l}
\sup _{k \in \mathbb{N}}\left\|f_{k}\right\|_{L^{p}\left(\mathbb{R}^{N}\right)}<\infty \text { and } \\
\lim _{k \rightarrow \infty} \breve{e}_{L^{p}}\left(f_{k}, f\right)=0 .
\end{array}\right.
$$

Proof The " $\Leftarrow "$ direction: This is an immediate consequence of the fact that $C_{c}^{1}\left(\mathbb{R}^{N}\right)$ is a dense subset of the dual space $L^{q}\left(\mathbb{R}^{N}\right)=\left(L^{p}\left(\mathbb{R}^{N}\right)\right)^{\prime}$ (see, e.g., [68, IV.9 Example 3]).

The " $\Rightarrow$ " direction: Every weakly converging sequence is known to be bounded w.r.t. the underlying norm as a consequence of the Banach-Steinhaus theorem. Write $S:=$ $\sup \left\|f_{k}\right\|_{L^{p}\left(\mathbb{R}^{N}\right)}<\infty$. It follows from the lower semicontinuity of the norm w.r.t. the $k \in \mathbb{N}$ weak convergence that $\|f\|_{L^{p}\left(\mathbb{R}^{N}\right)} \leq S$.

To prove that $\breve{e}_{L} p\left(f_{k}, f\right) \rightarrow 0$, choose $\varepsilon>0$ arbitrarily. There is a radius $\rho \geq 1$ such that

$$
\|f\|_{L^{p}\left(\mathbb{R}^{N} \backslash \mathbb{B}_{\rho}(0)\right)}+\sup _{k \in \mathbb{N}}\left\|f_{k}\right\|_{L^{p}\left(\mathbb{R}^{N} \backslash \mathbb{B}_{\rho}(0)\right)}<\frac{\varepsilon}{2}
$$

since $\left(\left|f_{k}\right|^{p}\right)_{k \in \mathbb{N}}$ is tight by assumption. According to the Sobolev embedding theorem, the set $\left\{\varphi \in C_{c}^{1}\left(\mathbb{B}_{2 \rho}(0)\right) \mid\|\varphi\|_{W^{1, q}} \leq 1,\|\varphi\|_{W^{1, \infty}} \leq 1\right\}$ (strictly speaking, their zero extensions to $\left.\mathbb{R}^{N}\right)$ is relatively compact in $\left(L^{q}\left(\mathbb{R}^{N}\right),\|\cdot\|_{L^{q}\left(\mathbb{R}^{N}\right)}\right)$. Hence there exist finitely many functions $\varphi_{1}, \ldots, \varphi_{j} \in C_{c}^{1}\left(\mathbb{B}_{2 \rho}(0)\right.$ ) (with $j=j(\varepsilon, \rho) \in \mathbb{N}$ ) such that $\sup _{i \leq j}\left\{\left\|\varphi_{i}\right\|_{W^{1, q}},\left\|\varphi_{i}\right\|_{W^{1, \infty}}\right\} \leq 1$ and $i \leq j$

$$
\begin{aligned}
& \left\{\varphi \in C_{c}^{1}\left(\mathbb{B}_{2 \rho}\right) \mid\|\varphi\|_{W^{1, q}} \leq 1,\|\varphi\|_{W^{1, \infty}} \leq 1\right\} \\
& \subset \bigcup_{i=1}^{j}\left\{g \in L^{q}\left(\mathbb{R}^{N}\right) \mid\left\|g-\varphi_{i}\right\|_{L^{q}}<\frac{\varepsilon}{4 S+1}\right\} .
\end{aligned}
$$

Then we obtain

$$
\begin{aligned}
\breve{e}_{L^{p}}\left(f_{k}, f\right) \stackrel{\text { Def. }}{=} \sup \left\{\int_{\mathbb{R}^{N}} \varphi \cdot\left(f_{k}-f\right) d \mathbf{x} \mid \varphi \in C_{c}^{1}\left(\mathbb{R}^{N}\right),\|\varphi\|_{W^{1, q}} \leq 1,\|\varphi\|_{W^{1, \infty}} \leq 1\right\} \\
\leq \sup \left\{\int_{\mathbb{R}^{N}} \varphi \cdot\left(f_{k}-f\right) d \mathbf{x} \mid \varphi \in C_{c}^{1}\left(\mathbb{B}_{2 \rho}\right),\|\varphi\|_{W^{1, q}} \leq 1,\|\varphi\|_{W^{1, \infty}} \leq 1\right\} \\
\quad+\frac{\varepsilon}{2}
\end{aligned}
$$




$$
\begin{aligned}
& \leq \sup _{1 \leq i \leq j} \int_{\mathbb{R}^{N}} \varphi_{i} \cdot\left(f_{k}-f\right) d \mathbf{x}+\frac{\varepsilon}{4 S+1}\left\|f_{k}-f\right\|_{L^{p}\left(\mathbb{R}^{N}\right)}+\frac{\varepsilon}{2} \\
& \leq \sup _{1 \leq i \leq j} \int_{\mathbb{R}^{N}} \varphi_{i} \cdot\left(f_{k}-f\right) d \mathbf{x}+\varepsilon .
\end{aligned}
$$

Hence $\lim \sup _{k \rightarrow \infty} \breve{e}_{L^{p}}\left(f_{k}, f\right)=0$.

Corollary 14 Every norm-bounded closed tight subset of $L^{p}\left(\mathbb{R}^{N}\right)$ is (sequentially) compact w.r.t. $\breve{e}_{L^{p}}$.

Proof The Banach space $L^{p}\left(\mathbb{R}^{N}\right)$ (with $1<p<\infty$ ) is reflexive and so, every bounded closed ball in $L^{p}\left(\mathbb{R}^{N}\right)$ is known to be sequentially compact with respect to the weak topology (e.g., [68, V.1.Theorem 1]). The equivalence in Lemma 13 then implies convergence w.r.t. $\breve{e}_{L^{p}}$.

Corollary 15 Let $\left(f_{k}\right)_{k \in \mathbb{N}}$ and $\left(g_{k}\right)_{k \in \mathbb{N}}$ be two bounded sequences in $L^{p}\left(\mathbb{R}^{N}\right)$ such that both $\left(\left|f_{k}\right|^{p}\right)_{k \in \mathbb{N}}$ and $\left(\left|g_{k}\right|^{p}\right)_{k \in \mathbb{N}}$ are tight in $\mathbb{R}^{N}$. Then the following equivalence holds

$$
\lim _{k \rightarrow \infty} \breve{e}_{L^{p}}\left(f_{k}, g_{k}\right)=0 \Leftrightarrow \lim _{k \rightarrow \infty} d_{L^{p}}\left(f_{k}, g_{k}\right)=0
$$

Proof The " $\Leftarrow$ " direction: This is a consequence of $\breve{e}_{L^{p}} \leq d_{L^{p}}$.

The " $\Rightarrow$ " direction: This implication results from essentially the same arguments as Lemma 13 for two reasons: Firstly, the (now larger) subset $\{\varphi \in$ $\left.C_{c}^{1}\left(\mathbb{B}_{2 \rho}(0)\right) \mid\|\varphi\|_{L^{q}} \leq 1,\|\varphi\|_{W^{1, \infty}} \leq 1\right\}$ is also relatively compact in $\left(L^{q}\left(\mathbb{R}^{N}\right), \|\right.$. $\left.\|_{L^{q}\left(\mathbb{R}^{N}\right)}\right)$. Secondly, both $d_{L^{p}}$ and $\breve{e}_{L^{p}}$ depend only on the difference of their two arguments. (These metrics originate from norms, although we do not use this deeper structure elsewhere.)

Corollary 16 Norm-bounded closed convex tight subsets of $L^{p}\left(\mathbb{R}^{N}\right)$ are relatively compact with respect to $d_{L^{p}}$ in the following sense: Let $M \subset L^{p}\left(\mathbb{R}^{N}\right)$ be any normbounded closed convex subset with $\lim _{\rho \rightarrow \infty} \sup _{f \in M}\|f\|_{L^{p}\left(\mathbb{R}^{N} \backslash \mathbb{B}_{\rho}(0)\right)}=0$. Then every sequence in $M$ has a subsequence converging in $\left(L^{p}\left(\mathbb{R}^{N}\right), d_{L^{p}}\right)$.

Proposition 17 The following equivalence holds for any sequence $\left(f_{k}\right)_{k \in \mathbb{N}}$ in $L^{p}\left(\mathbb{R}^{N}\right)$ :

$$
\begin{aligned}
& \lim _{k \rightarrow \infty}\left\|f_{k}-f\right\|_{L^{p}\left(\mathbb{R}^{N}\right)}=0 \Leftrightarrow\left\{\begin{array}{l}
\lim _{k \rightarrow \infty} e_{L^{p}}\left(f_{k}, f\right)=0 \\
\left(\left|f_{k}\right|^{p}\right)_{k \in \mathbb{N}} \text { is tight in } \mathbb{R}^{N}
\end{array}\right. \\
& \Leftrightarrow\left\{\begin{array}{l}
\lim _{k \rightarrow \infty}\left\|f_{k}\right\|_{L^{p}}=\|f\|_{L^{p}} \\
\lim _{k \rightarrow \infty} d_{L^{p}}\left(f_{k}, f\right)=0 \\
\left(\left|f_{k}\right|^{p}\right)_{k \in \mathbb{N}} \text { is tight in } \mathbb{R}^{N} .
\end{array}\right.
\end{aligned}
$$


Proof This results essentially from the fact that $L^{p}\left(\mathbb{R}^{N}\right)$ is a reflexive Banach space. According to a theorem by Riesz and Radon (e.g., [3, U6.6] or [34, Proposition 5.10]), every weakly converging sequence $\left(f_{k}\right)_{k \in \mathbb{N}}$ in $L^{p}\left(\mathbb{R}^{N}\right)$ has the property $\| f_{k}-$ $f\left\|_{L^{p}\left(\mathbb{R}^{N}\right)} \rightarrow 0(k \rightarrow \infty) \quad \Leftrightarrow\right\| f_{k}\left\|_{L^{p}\left(\mathbb{R}^{N}\right)} \rightarrow\right\| f \|_{L^{p}\left(\mathbb{R}^{N}\right)}(k \rightarrow \infty)$. Hence the first claimed equivalence is a direct consequence of Lemma 13. Finally the second equivalence results from Remark 11.

Proposition 18 Norm-bounded closed convex tight subsets of $L^{p}\left(\mathbb{R}^{N}\right)$ are complete with respect to $d_{L^{p}}$ in the following sense: Let $M \subset L^{p}\left(\mathbb{R}^{N}\right)$ be any norm-bounded closed convex subset with $\lim _{\rho \rightarrow \infty} \sup _{f \in M}\|f\|_{L^{p}\left(\mathbb{R}^{N} \backslash \mathbb{B}_{\rho}(0)\right)}=0$. Then every Cauchy sequence w.r.t. $d_{L^{p}}$ in $M$ has a limit in $M$ w.r.t. $d_{L^{p}}$.

This can be concluded indirectly from the compactness property in Corollary 16. Supplementarily we now give a separate proof without using the topological equivalence of $d_{L^{p}}$ and $e_{L^{p}}$ specified in Corollary 15 (see [54, Section 5.1] or [53, Section 5.1] for the corresponding metrics on $L^{2}$ with different arguments).

Proof Let $\left(f_{k}\right)_{k \in \mathbb{N}}$ be a Cauchy sequence in $M$ w.r.t. $d_{L^{p}}$. Then $\left(f_{k}\right)_{k \in \mathbb{N}}$ is Cauchy sequence w.r.t. $\breve{e}_{L^{p}}$ due to Remark 11 . Hence Corollary 14 provides a function $f \in$ $L^{p}\left(\mathbb{R}^{N}\right)$ with $\breve{e}_{L^{p}}\left(f_{k}, f\right) \rightarrow 0$ as $k \rightarrow \infty$ or equivalently $f_{k} \rightarrow f$ weakly in $L^{p}\left(\mathbb{R}^{N}\right)$. By Mazur's Lemma (see, e.g., [68, V.1.Theorem 2]), the norm-closed convex set $M \subset L^{p}\left(\mathbb{R}^{N}\right)$ is weakly closed, so $f \in M$.

It remains to prove $d_{L^{p}}\left(f_{k}, f\right) \rightarrow 0$ for $k \rightarrow \infty$. Choose any $\varepsilon>0$. As $\left(f_{k}\right)_{k \in \mathbb{N}}$ is Cauchy sequence w.r.t. $d_{L^{p}}$, there is some $J=J(\varepsilon) \in \mathbb{N}$ with $d_{L^{p}}\left(f_{k}, f_{l}\right) \leq \varepsilon$ for all $k, l \geq J$. For every test function $\varphi \in C_{c}^{1}\left(\mathbb{R}^{N}\right)$ with $\|\varphi\|_{L^{q}} \leq 1,\|\varphi\|_{L^{\infty}} \leq$ $1,\left\|\nabla_{\mathbf{x}} \varphi\right\|_{L^{\infty}} \leq 1$ and any $k \geq J$, we conclude from the weak convergence of $\left(f_{l}\right)_{l \in \mathbb{N}}$ to $f$ that

$\int_{\mathbb{R}^{N}} \varphi \cdot\left(f_{k}-f\right) d \mathbf{x}=\limsup _{l \rightarrow \infty} \int_{\mathbb{R}^{N}} \varphi \cdot\left(f_{k}-f_{l}\right) d \mathbf{x} \leq \limsup _{l \rightarrow \infty} d_{L^{p}}\left(f_{k}, f_{l}\right) \leq \varepsilon$, i.e., $d_{L^{p}}\left(f_{k}, f\right) \leq \varepsilon$ holds for every $k \geq J$.

\subsection{Nonautonomous linear problem: uniqueness of weak solutions}

Proposition 19 Suppose for any $T>0$ that $\widetilde{\mathbf{g}} \in L^{\infty}\left(0, T ; L^{\infty}\left(\mathbb{R}^{N}, \mathbb{R}^{N}\right)\right), \partial_{\mathbf{x}} \widetilde{\mathbf{g}} \in$ $L^{\infty}\left(0, T ; L^{\infty}\left(\mathbb{R}^{N}, \mathbb{R}^{N \times N}\right)\right), \tilde{u} \in L^{\infty}\left(0, T ; L^{\infty}\left(\mathbb{R}^{N}\right)\right)$ and $\widetilde{w} \in L^{1}\left(0, T ; L^{p}\left(\mathbb{R}^{N}\right)\right)$. Then for any initial function $f_{0} \in L^{p}\left(\mathbb{R}^{N}\right)$, there exists at most one weak solution $f \in L^{1}\left(0, T ; L^{p}\left(\mathbb{R}^{N}\right)\right)$ of the nonautonomous transport equation in $[0, T]$

$$
\partial_{t} f+\operatorname{div}_{\mathbf{x}}(f \widetilde{\mathbf{g}}(t))=\widetilde{u}(t) f+\widetilde{w}(t), \quad f(0)=f_{0} .
$$

In short, every weak solution in Proposition 19 proves to be a "renormalized" solution (in the sense of DiPerna and Lions [33]) and so is unique. The proof is essentially based on the smoothing arguments of Le Bris and Lions in their article 
[48] about "transport equations with partially $W^{1,1}$ velocities". The norms in $L^{1}, L^{\infty}$ used there have just to be replaced by the $L^{p}$ norm as indicated in [33].

Weak (possibly measure-valued) solutions to linear transport equations and the relation to their (generalised) flow along ODEs (with discontinuous coefficients) belong to the current fields of research in analysis (see, e.g., [4-6, 17,55,56] and references therein). Existence of solutions can usually be proved by means of smoothing coefficients. Uniqueness of weak solutions, however, proves to be a very challenging topic. [55, Theorem 5.10] exemplifies how rather weak assumptions about the divergence of the vector fields ensure the uniqueness of weak solutions to homogeneous transport equations if their values are nonnegative bounded Radon measures on the Euclidean space. In [6], however, it is pointed out as an open question whether similar uniqueness results also hold for signed measures (as values of weak solutions), unless the vector field is assumed to fulfil an Osgood-type condition.

\subsection{Linear problem with "more regular" coefficients: existence of solutions}

Proposition 20 Suppose that $\mathbf{g} \in C_{c}^{1}\left(\mathbb{R}^{N}, \mathbb{R}^{N}\right), u \in C^{1}\left(\mathbb{R}^{N}\right) \cap W^{1, \infty}\left(\mathbb{R}^{N}\right)$ and $w \in L^{p}\left(\mathbb{R}^{N}\right)$. Then for any initial function $f_{0} \in L^{p}\left(\mathbb{R}^{N}\right)$ and period $T>0$, there exists a weak solution $f:[0, T] \rightarrow L^{p}\left(\mathbb{R}^{N}\right)$ of

$$
\partial_{t} f+\operatorname{div}_{\mathbf{x}}(f \mathbf{g})=u f+w, \quad f(0)=f_{0}
$$

in the sense that $f:[0, T] \rightarrow L^{p}\left(\mathbb{R}^{N}\right)$ is weakly continuous with $f(0)=f_{0}$ and

$$
\begin{aligned}
\int_{\mathbb{R}^{N}} \varphi\left(f\left(t_{2}\right)-f\left(t_{1}\right)\right) d \mathbf{x}= & \int_{t_{1}}^{t_{2}} \int_{\mathbb{R}^{N}}\left(f(s, \cdot)\left(\mathbf{g} \cdot \nabla_{\mathbf{x}} \varphi+u \varphi\right)\right) d \mathbf{x} d s \\
& +\left(t_{2}-t_{1}\right) \int_{\mathbb{R}^{N}} \varphi w d \mathbf{x}
\end{aligned}
$$

for any $0 \leq t_{1}<t_{2} \leq T, \varphi \in C_{c}^{1}\left(\mathbb{R}^{N}\right)$.

This solution can be represented as

$$
\int_{\mathbb{R}^{N}} \varphi \cdot f(t) d \mathbf{x}=\int_{\mathbb{R}^{N}}\left(\psi_{t, \varphi}(0 ; \cdot) f_{0}+w \int_{0}^{t} \psi_{t, \varphi}(s ; \cdot) d s\right) d \mathbf{x}
$$

with $\psi_{t, \varphi} \in C^{1}\left([0, t] \times \mathbb{R}^{N}\right)$ denoting the unique solution to the adjoint problem (11) below. Moreover, with the notation $[r]^{-}:=\min \{r, 0\}$ for $r \in \mathbb{R}$, it satisfies the a priori estimate

$$
\|f(t)\|_{L^{p}\left(\mathbb{R}^{N}\right)} \leq\left(\left\|f_{0}\right\|_{L^{p}\left(\mathbb{R}^{N}\right)}+\|w\|_{L^{p}\left(\mathbb{R}^{N}\right)} t\right) \cdot e^{\left(\left\|\left[\operatorname{div}_{\mathbf{x}} \mathbf{g}\right]^{-}\right\|_{L^{\infty}}+\|u\|_{L^{\infty}}\right) \cdot t} .
$$

The proof is given at the end of the subsection after some preparatory lemmata.

The characterisation of "weak solution" in Proposition 20 differs slightly from the standard definition, but it implies successively by means of approximation that: 
1. For any $\varphi \in C_{c}^{1}\left(\mathbb{R}^{N}\right)$, the function from $[0, T]$ to $\mathbb{R}$ given by $t \mapsto \int_{\mathbb{R}^{N}} \varphi f(t) d \mathbf{x}$ is Lipschitz continuous. Indeed the image $f([0, T]) \subset L^{p}\left(\mathbb{R}^{N}\right)$ is weakly compact, hence bounded and so, the claimed Lipschitz continuity results from the detailed reformulation (6) of weak solutions.

2. In addition, for any test function $\phi \in C_{c}^{\infty}\left([0, T] \times \mathbb{R}^{N}\right)$ depending on time

$$
\begin{aligned}
& \int_{\mathbb{R}^{N}}\left(\phi(T, \mathbf{x}) f(T, \mathbf{x})-\phi(0, \mathbf{x}) f_{0}(\mathbf{x})\right) d \mathbf{x} \\
&= \int_{0}^{T} \int_{\mathbb{R}^{N}}\left(f(s, \mathbf{x})\left(\mathbf{g}(\mathbf{x}) \cdot \nabla_{\mathbf{x}} \phi(s, \mathbf{x})+u(\mathbf{x}) \phi(s, \mathbf{x})\right)\right) d \mathbf{x} d s \\
& \quad+\int_{0}^{T} \int_{\mathbb{R}^{N}}\left(\phi(s, \mathbf{x}) w+\partial_{s} \phi(s, \mathbf{x}) f(s, \mathbf{x})\right) d \mathbf{x} d s .
\end{aligned}
$$

The existence of weak solutions in $L^{\infty}\left(0, T ; L^{p}\left(\mathbb{R}^{N}\right)\right)$ of the initial value problem (5), has already been verified under weaker assumptions about $\mathbf{g}, \operatorname{div}_{\mathbf{x}} \mathbf{g}$ and $u$ (see, e.g., [33, Proposition II.1] with the modified regularization presented in [48]). For investigating the dependence of solutions on initial data and coefficients, however, we prefer supplementary uniqueness and need an appropriate representation such as in $(7)$.

Lemma 21 (The flow along the autonomous ODE) For every $\alpha \in \mathbb{R}$ and $\mathbf{g} \in$ $L_{\mathrm{loc}}^{1}\left(\mathbb{R}^{N}, \mathbb{R}^{N}\right)$ with $\partial_{\mathbf{x}} \mathbf{g} \in L^{\infty}\left(\mathbb{R}^{N}, \mathbb{R}^{N \times N}\right)$ there exists a function $\mathfrak{X}_{\alpha, \mathbf{g}}:[0, \infty) \times$ $\mathbb{R}^{N} \rightarrow \mathbb{R}^{N}$ induced by the unique Carathéodory solutions to the autonomous ordinary differential equation

$$
\partial_{t} \mathfrak{X}_{\alpha, \mathbf{g}}(t ; \mathbf{x})=\alpha \cdot \mathbf{g}\left(\mathfrak{X}_{\alpha, \mathbf{g}}(t ; \mathbf{x})\right), \quad \mathfrak{X}_{\alpha, \mathbf{g}}(0 ; \mathbf{x})=\mathbf{x} .
$$

Moreover, $\mathfrak{X}_{\alpha, \mathbf{g}}(t ; \cdot): \mathbb{R}^{N} \rightarrow \mathbb{R}^{N}$ is a Lipschitz continuous homeomorphism for each $t \geq 0$ and, with the notation $[r]^{+}:=\max \{r, 0\},[r]^{-}:=\min \{r, 0\}$ for $r \in \mathbb{R}$, satisfies

$$
\begin{aligned}
\left\|\partial_{\mathbf{x}} \mathfrak{X}_{\alpha, \mathbf{g}}(t ; \cdot)\right\|_{L^{\infty}\left(\mathbb{R}^{N}, \mathbb{R}^{N \times N}\right)} & \leq e^{\operatorname{const}(N,|\alpha|)\left(1+\left\|\partial_{\mathbf{x}} \mathbf{g}\right\|_{L} \infty\right) \cdot t} \\
\frac{\partial}{\partial t} \operatorname{det} \partial_{\mathbf{x}} \mathfrak{X}_{\alpha, \mathbf{g}}(t ; \cdot) & =\alpha \cdot\left(\operatorname{div}_{\mathbf{x}} \mathbf{g}\right)\left(\mathfrak{X}_{\alpha, \mathbf{g}}(t ; \cdot)\right) \cdot \operatorname{det} \partial_{\mathbf{x}} \mathfrak{X}_{\alpha, \mathbf{g}}(t ; \cdot) \\
e^{-|\alpha|\left\|\left[\operatorname{div}_{\mathbf{x}} \mathbf{g}\right]^{-}\right\|_{L^{\infty}} \cdot t} & \leq \operatorname{det} \partial_{\mathbf{x}} \mathfrak{X}_{\alpha, \mathbf{g}}(t ; \cdot) \leq e^{|\alpha|\left\|\left[\operatorname{div}_{\mathbf{x}} \mathbf{g}\right]^{+}\right\|_{L^{\infty} \cdot t}}
\end{aligned}
$$

If, in addition, $\mathbf{g} \in C_{c}^{1}\left(\mathbb{R}^{N}, \mathbb{R}^{N}\right)$, then $\mathfrak{X}_{\alpha, \mathbf{g}}(t, \cdot): \mathbb{R}^{N} \rightarrow \mathbb{R}^{N}$ is continuously differentiable for each $t \in[0, \infty)$ and satisfies

$$
\left\|\partial_{\mathbf{x}} \mathfrak{X}_{\alpha, \mathbf{g}}(t ; \cdot)\right\|_{L^{\infty}\left(\mathbb{R}^{N}, \mathbb{R}^{N \times N}\right)} \leq e^{\operatorname{const}(N,|\alpha|)\left(1+\|D \mathbf{g}\|_{L^{\infty}}\right) \cdot t} .
$$

In particular, each function $\mathfrak{X}_{\alpha, \mathbf{g}}(t, \cdot): \mathbb{R}^{N} \rightarrow \mathbb{R}^{N}$ for $t \in[0, \infty)$, is a diffeomorphism.

These properties of $\mathfrak{X}_{\alpha, \mathbf{g}}$ result from the standard theory about ordinary differential equations (see, e.g., [39, § V.3], [4, Remark 6.3]). 
Lemma 22 Suppose that $\mathbf{g} \in C_{c}^{1}\left(\mathbb{R}^{N}, \mathbb{R}^{N}\right)$ and $u \in C^{1}\left(\mathbb{R}^{N}\right) \cap W^{1, \infty}\left(\mathbb{R}^{N}\right)$. Then for any $t \in[0, T]$ and $\varphi \in C_{c}^{1}\left(\mathbb{R}^{N}\right)$, there is a unique solution $\psi_{t, \varphi}=\psi \in C^{1}([0, t] \times$ $\mathbb{R}^{N}$ ) of the first-order end-time problem

$$
\partial_{s} \psi+\mathbf{g} \cdot \nabla_{\mathbf{x}} \psi+u \psi=0 \text { in }[0, t] \times \mathbb{R}^{N}, \quad \psi(t)=\varphi \text { in } \mathbb{R}^{N}
$$

given by

$$
\psi(s ; \mathbf{x})=\varphi\left(\mathfrak{X}_{1, \mathbf{g}}(t-s ; \mathbf{x})\right) \cdot \exp \left(\int_{0}^{t-s} u\left(\mathfrak{X}_{1, \mathbf{g}}(r ; \mathbf{x})\right) d r\right) .
$$

Moreover, the a priori estimate

$$
\left\|\psi_{t, \varphi}(s ; \cdot)\right\|_{L^{q}} \leq\|\varphi\|_{L^{q}} \cdot e^{\left(\left\|\left[\operatorname{div}_{\mathbf{x}} \mathbf{g}\right]^{-}\right\|_{\left.L^{\infty}+\|u\|_{L^{\infty}}\right) \cdot t}\right.}
$$

holds for every $0 \leq s \leq t \leq T$ and $\varphi \in C_{c}^{1}\left(\mathbb{R}^{n}\right)$.

Proof Choose $t \in[0, T]$ and $\varphi \in C_{c}^{1}\left(\mathbb{R}^{N}\right)$. The method of characteristics provides the explicit solution $\phi \in C^{1}\left([0, t] \times \mathbb{R}^{N}\right)$, namely

$$
\phi(s ; \cdot)=\varphi\left(\mathfrak{X}_{1, \mathbf{g}}(s ; \cdot)\right) \cdot \exp \left(\int_{0}^{s} u\left(\mathfrak{X}_{1, \mathbf{g}}(s-r ; \cdot)\right) d r\right)
$$

of the semi-linear initial value problem

$$
\partial_{s} \phi-\mathbf{g} \cdot \nabla_{\mathbf{x}} \phi-u \phi=0 \quad \text { in }[0, t] \times \mathbb{R}^{N}, \quad \phi(0)=\varphi \text { in } \mathbb{R}^{N} .
$$

Substituting $\psi(\tau ; \mathbf{x}):=\phi(t-\tau ; \mathbf{x})$ for $\tau \in[0, t]$, we obtain the claimed solution $\psi \in C^{1}\left([0, t] \times \mathbb{R}^{N}\right)$ of the given transport equation with end-time condition $\psi(t, \cdot)=$ $\varphi$ :

$$
\psi(s ; \cdot)=\varphi\left(\mathfrak{X}_{1, \mathbf{g}}(t-s ; \cdot)\right) \cdot \exp \left(\int_{s}^{t} u\left(\mathfrak{X}_{1, \mathbf{g}}(r-s ; \cdot)\right) d r\right) .
$$

Moreover, the transformation theorem for Lebesgue integrals leads to

$$
\begin{aligned}
\int_{\mathbb{R}^{N}}\left|\psi_{t, \varphi}(s ; \mathbf{x})\right|^{q} d \mathbf{x} & \leq e^{q\|u\|_{L^{\infty} \cdot(t-s)}} \cdot \int_{\mathbb{R}^{N}}\left|\varphi\left(\mathfrak{X}_{1, \mathbf{g}}(t-s ; \mathbf{x})\right)\right|^{q} d \mathbf{x} \\
& \leq e^{q\|u\|_{L^{\infty} \cdot(t-s)}} \cdot\left\|\operatorname{det}\left(\partial_{\mathbf{x}} \mathfrak{X}_{1, \mathbf{g}}(t-s ; \cdot)\right)^{-1}\right\|_{L^{\infty}}\|\varphi\|_{L^{q}}^{q} \\
& \leq e^{q\|u\|_{L^{\infty} \cdot t}} \cdot e^{\left\|\left[\operatorname{div}_{\mathbf{x}} \mathbf{g}\right]^{-}\right\|_{L^{\infty}} \cdot t}\|\varphi\|_{L^{q}}^{q} \cdot
\end{aligned}
$$


Proof of Proposition 20 For any $t \in[0, T]$ and $\varphi \in C_{c}^{1}\left(\mathbb{R}^{N}\right)$, consider the solution $\psi_{t, \varphi} \in C^{1}\left([0, t] \times \mathbb{R}^{N}\right)$ to the semi-linear end-time problem (11). Due to the a priori bound (13) in Lemma 22, the linear operators

$$
\begin{aligned}
\varphi \mapsto \psi_{t, \varphi}(s ; \cdot)=\varphi\left(\mathfrak{X}_{1, \mathbf{g}}(t-s ; \cdot)\right) \cdot \exp \left(\int_{0}^{t-s} u\left(\mathfrak{X}_{1, \mathbf{g}}(r ; \cdot)\right) d r\right), \\
\varphi \mapsto \int_{0}^{t} \psi_{t, \varphi}(s ; \cdot) d s
\end{aligned}
$$

from $C_{c}^{1}\left(\mathbb{R}^{N}\right)$ into $C^{1}\left(\mathbb{R}^{N}\right)$ are continuous with respect to the $L^{q}\left(\mathbb{R}^{N}\right)$ norm and so, they have unique continuous extensions to $L^{q}\left(\mathbb{R}^{N}\right)$.

For each $t \in[0, T]$, select the unique function $f(t)$ in the dual space $L^{p}\left(\mathbb{R}^{N}\right)$ of $L^{q}\left(\mathbb{R}^{N}\right)$ such that

$$
\int_{\mathbb{R}^{N}} \varphi f(t) d \mathbf{x}=\int_{\mathbb{R}^{N}}\left(\psi_{t, \varphi}(0 ; \cdot) f_{0}+w \int_{0}^{t} \psi_{t, \varphi}(s ; \cdot) d s\right) d \mathbf{x}
$$

for any $\varphi \in C_{c}^{1}\left(\mathbb{R}^{N}\right)$. In particular, $\|f(t)\|_{L^{p}\left(\mathbb{R}^{N}\right)}$ is bounded by a constant depending on $\left\|\left[\operatorname{div}_{\mathbf{x}} \mathbf{g}\right]^{-}\right\|_{L^{\infty}},\|u\|_{L^{\infty}},\|w\|_{L^{p}},\left\|f_{0}\right\|_{L^{p}}$ and $T$ only:

$$
\begin{aligned}
& \|f(t)\|_{L^{p}}=\sup \left\{\int_{\mathbb{R}^{N}} \varphi \cdot f(t) d \mathbf{x} \mid \varphi \in L^{q}\left(\mathbb{R}^{N}\right),\|\varphi\|_{L^{q}} \leq 1\right\} \\
& =\sup \left\{\int_{\mathbb{R}^{N}} \varphi \cdot f(t) d \mathbf{x} \mid \varphi \in C_{c}^{1}\left(\mathbb{R}^{N}\right),\|\varphi\|_{L^{q}} \leq 1\right\} \\
& =\sup \left\{\left\|\psi_{t, \varphi}(s ; \cdot)\right\|_{L^{q}}\left(\left\|f_{0}\right\|_{L^{p}}+\|w\|_{L^{p}} t\right) \mid \varphi \in C_{c}^{1}\left(\mathbb{R}^{N}\right)\right. \text {, } \\
& \left.\|\varphi\|_{L^{q}} \leq 1, \quad s \leq t\right\} \\
& \stackrel{(13)}{\leq} e^{\left(\left\|\left[\operatorname{div}_{\mathbf{x}} \mathbf{g}\right]^{-}\right\|_{L^{\infty}}+\|u\|_{\left.L^{\infty}\right) \cdot t}\right.} \cdot\left(\left\|f_{0}\right\|_{L^{p}\left(\mathbb{R}^{N}\right)}+\|w\|_{L^{p}\left(\mathbb{R}^{N}\right)} t\right) \text {. }
\end{aligned}
$$

This leads to the bounded function $f:[0, T] \rightarrow L^{p}\left(\mathbb{R}^{N}\right)$, which we now prove to be a weak solution of problem (5).

For any test function $\varphi \in C_{c}^{1}\left(\mathbb{R}^{N}\right)$ with compact support, the function $\Phi:[0, T] \rightarrow$ $\mathbb{R}$ given by $t \mapsto \int_{\mathbb{R}^{N}} \varphi f(t) d \mathbf{x}$ is absolutely continuous with

$$
\begin{aligned}
\Phi^{\prime}(t)= & \frac{d}{d t} \int_{\mathbb{R}^{N}}\left(f_{0}(\mathbf{x}) \varphi\left(\mathfrak{X}_{1, \mathbf{g}}(t ; \mathbf{x})\right) e^{\int_{0}^{t} u\left(\mathfrak{X}_{1, \mathbf{g}}(r ; \mathbf{x})\right) d r}\right. \\
& \left.+w(\mathbf{x}) \int_{0}^{t} \psi_{t, \varphi}(s ; \mathbf{x}) d s\right) d \mathbf{x} \\
= & \int_{\mathbb{R}^{N}} \frac{\partial}{\partial t}\left(f_{0}(\mathbf{x}) \varphi\left(\mathfrak{X}_{1, \mathbf{g}}(t ; \mathbf{x})\right) \cdot \exp \left(\int_{0}^{t} u\left(\mathfrak{X}_{1, \mathbf{g}}(r ; \mathbf{x})\right) d r\right)\right) d \mathbf{x} \\
& +\int_{\mathbb{R}^{N}} w(\mathbf{x}) \cdot \frac{\partial}{\partial t} \int_{0}^{t}\left(\varphi\left(\mathfrak{X}_{1, \mathbf{g}}(t-s ; \mathbf{x})\right) \cdot e^{\int_{0}^{t-s} u\left(\mathfrak{X}_{1, \mathbf{g}}(r ; \mathbf{x})\right) d r}\right) d s d \mathbf{x}
\end{aligned}
$$




$$
\begin{aligned}
= & \int_{\mathbb{R}^{N}}\left\{f _ { 0 } ( \mathbf { x } ) \left(\nabla \varphi\left(\mathfrak{X}_{1, \mathbf{g}}(t ; \mathbf{x})\right) \cdot \partial_{t} \mathfrak{X}_{1, \mathbf{g}}(t ; \mathbf{x})\right.\right. \\
& \left.\left.+\varphi\left(\mathfrak{X}_{1, \mathbf{g}}(t ; \mathbf{x})\right) u\left(\mathfrak{X}_{1, \mathbf{g}}(t ; \mathbf{x})\right)\right) \cdot \exp \left(\int_{0}^{t} u\left(\mathfrak{X}_{1, \mathbf{g}}(r ; \mathbf{x})\right) d r\right)\right\} d \mathbf{x} \\
& +\int_{\mathbb{R}^{N}} w(\mathbf{x})\left\{\varphi(\mathbf{x})+\int_{0}^{t}\left(\left.\nabla \varphi\right|_{\left(\mathfrak{X}_{1, \mathbf{g}}(t-s ; \mathbf{x})\right)} \cdot \partial_{t} \mathfrak{X}_{1, \mathbf{g}}(t-s ; \mathbf{x})\right.\right. \\
& \left.\left.+\left.\varphi\right|_{\left(\mathfrak{X}_{1, \mathbf{g}}(t-s ; \mathbf{x})\right)} u\left(\mathfrak{X}_{1, \mathbf{g}}(t-s ; \mathbf{x})\right)\right) e^{\int_{0}^{t-s} u\left(\mathfrak{X}_{1, \mathbf{g}}(r ; \mathbf{x})\right) d r} d s\right\} d \mathbf{x} \\
\stackrel{(14)}{=} & \int_{\mathbb{R}^{N}}(\nabla \varphi \cdot \mathbf{g}(\mathbf{x})+\varphi u) \cdot f(t) d \mathbf{x}+\int_{\mathbb{R}^{N}} \varphi w d \mathbf{x} .
\end{aligned}
$$

Indeed, the global a priori bound on both $\|f(t)\|_{L^{p}}$ and the other functions $\mathbf{g}, u, \varphi, w$ ensure that all Lebesgue integrals here exist and so, the rules of differentiation can be applied to weak derivatives (see, e.g., [69]).

\subsection{Linear problem with "more regular" coefficients: an explicit formula for solutions in $L^{p}\left(\mathbb{R}^{N}\right)$}

Proposition 23 Under the assumptions of Proposition 20, the weak solution $f$ : $[0, T] \rightarrow L^{p}\left(\mathbb{R}^{N}\right)$ of the initial value problem (5) presented in Eq. (7) has the explicit form

$$
\begin{aligned}
f(t, \xi)= & f_{0}\left(\mathfrak{X}_{-1, \mathbf{g}}(t ; \xi)\right) \cdot \exp \left(\int_{0}^{t}\left(u-\operatorname{div}_{\mathbf{x}} \mathbf{g}\right)\left(\mathfrak{X}_{-1, \mathbf{g}}(s ; \xi)\right) d s\right) \\
& +\int_{0}^{t}\left(w\left(\mathfrak{X}_{-1, \mathbf{g}}(t-s ; \xi)\right) \cdot \exp \left(\int_{0}^{t-s}\left(u-\operatorname{div}_{\mathbf{x}} \mathbf{g}\right)\left(\mathfrak{X}_{-1, \mathbf{g}}(r ; \xi)\right) d r\right)\right) d s .
\end{aligned}
$$

For our conclusions later on, the main benefit of this explicit formula is to provide a dominating function of space and time. It will help us to verify tightness of solutions while coefficients are perturbed in a suitably bounded way (see Lemma 31 below).

Corollary 24 (A function dominating this weak solution) Under the assumptions of Proposition 20, the weak solution $f:[0, T] \rightarrow L^{p}\left(\mathbb{R}^{N}\right)$ of initial value problem (5) presented in Eq. (7) satisfies

$$
\begin{aligned}
& |f(t, \xi)| \leq e^{\left(\left\|\left[\operatorname{div}_{\mathbf{x}} \mathbf{g}\right]^{-}\right\|_{L^{\infty}}+\|u\|_{L^{\infty}}\right) \cdot t} \cdot\left(\left|f_{0}\left(\mathfrak{X}_{-1, \mathbf{g}}(t ; \xi)\right)\right|\right. \\
& \left.+\int_{0}^{t}\left|w\left(\mathfrak{X}_{-1, \mathbf{g}}(t-s ; \xi)\right)\right| d s\right), \\
& |f(t, \xi)|^{p} \leq C e^{C t} \cdot\left(\left|f_{0}\left(\mathfrak{X}_{-1, \mathbf{g}}(t ; \xi)\right)\right|^{p}+\int_{0}^{t}\left|w\left(\mathfrak{X}_{-1, \mathbf{g}}(t-s ; \xi)\right)\right|^{p} d s\right)
\end{aligned}
$$

for every $t \in[0, T]$ and Lebesgue-almost every $\xi \in \mathbb{R}^{N}$ with a finite constant $C$ that depends on $N, p, T,\left\|\left[\operatorname{div}_{\mathbf{x}} \mathbf{g}\right]^{-}\right\|_{L^{\infty}}$ and $\|u\|_{L^{\infty}}$. 
Proof of Proposition 23 The main idea is to change the right-hand side of Eq. (7) by means of the theorems of Fubini and the change of variables. The change of coordinates $\mathbf{x} \mapsto \mathfrak{X}_{1, \mathbf{g}}(t ; \mathbf{x})=\xi$ from $\mathbb{R}^{N}$ to $\mathbb{R}^{N}$ has the continuously differentiable inverse $\xi \mapsto \mathfrak{X}_{-1, \mathbf{g}}(t ; \xi)=\mathbf{x}$ from $\mathbb{R}^{N}$ to $\mathbb{R}^{N}$. Hence, the change of variables formula for Lebesgue integrals (see, e.g., [47, XXI, Theorem 2.6]) leads to

$$
\begin{aligned}
\int_{\mathbb{R}^{N}} & \psi_{t, \varphi}(0 ; \mathbf{x}) f_{0}(\mathbf{x}) d \mathbf{x} \\
\stackrel{(12)}{=} & \int_{\mathbb{R}^{N}}\left\{\varphi\left(\mathfrak{X}_{1, \mathbf{g}}(t ; \mathbf{x})\right) \cdot \exp \left(\int_{0}^{t} u\left(\mathfrak{X}_{1, \mathbf{g}}(r ; \mathbf{x})\right) d r\right)\right\} f_{0}(\mathbf{x}) d \mathbf{x} \\
= & \int_{\mathbb{R}^{N}} \varphi(\xi) \cdot \exp \left(\int_{0}^{t} u\left(\mathfrak{X}_{1, \mathbf{g}}\left(r ; \mathfrak{X}_{-1, \mathbf{g}}(t ; \xi)\right)\right) d r\right) f_{0}\left(\mathfrak{X}_{-1, \mathbf{g}}(t ; \xi)\right) \\
& \cdot\left|\operatorname{det} \partial_{\xi} \mathfrak{X}_{-1, \mathbf{g}}(t ; \xi)\right| d \xi \\
= & \int_{\mathbb{R}^{N}} \varphi(\xi) \cdot \exp \left(\int_{0}^{t} u\left(\mathfrak{X}_{-1, \mathbf{g}}(t-r ; \xi) d r\right) f_{0}\left(\mathfrak{X}_{-1, \mathbf{g}}(t ; \xi)\right)\right. \\
& \cdot\left|\operatorname{det} \partial_{\xi} \mathfrak{X}_{-1, \mathbf{g}}(t ; \xi)\right| d \xi \\
\stackrel{(10)}{=} & \int_{\mathbb{R}^{N}} \varphi(\xi) \cdot \exp \left(\int_{0}^{t} u\left(\mathfrak{X}_{-1, \mathbf{g}}(t-r ; \xi) d r\right) f_{0}\left(\mathfrak{X}_{-1, \mathbf{g}}(t ; \xi)\right)\right. \\
& \cdot e^{-} \int_{0}^{t}\left(\operatorname{div}_{\mathbf{x}} \mathbf{g}\right)\left(\mathfrak{X}_{-1, \mathbf{g}}(s ; \xi)\right) d s{ }_{\xi} \\
= & \int_{\mathbb{R}^{N}} \varphi(\xi) \cdot \exp \left(\int_{0}^{t}\left(u-\operatorname{div}_{\mathbf{x}} \mathbf{g}\right)\left(\mathfrak{X}_{-1, \mathbf{g}}(s ; \xi)\right) d s\right) f_{0}\left(\mathfrak{X}_{-1, \mathbf{g}}(t ; \xi)\right) d \xi .
\end{aligned}
$$

Similarly, by the transformation $\mathbf{x} \mapsto \mathfrak{X}_{1, \mathbf{g}}(t-s ; \mathbf{x})=\xi$, we obtain

$$
\begin{aligned}
\int_{\mathbb{R}^{N}}\left(w(\mathbf{x}) \cdot \int_{0}^{t} \psi_{t, \varphi}(s ; \mathbf{x}) d s\right) d \mathbf{x} \\
\stackrel{(10)}{=} \int_{0}^{t} \int_{\mathbb{R}^{N}} w(\mathbf{x}) \cdot \varphi\left(\mathfrak{X}_{1, \mathbf{g}}(t-s ; \mathbf{x})\right) \cdot \exp \left(\int_{0}^{t-s} u\left(\mathfrak{X}_{1, \mathbf{g}}(r ; \mathbf{x})\right) d r\right) d \mathbf{x} d s \\
=\int_{0}^{t} \int_{\mathbb{R}^{N}} w\left(\mathfrak{X}_{-1, \mathbf{g}}(t-s ; \xi)\right) \cdot \varphi(\xi) \\
\cdot \exp \left(\int_{0}^{t-s} u\left(\mathfrak{X}_{1, \mathbf{g}}\left(r ; \mathfrak{X}_{-1, \mathbf{g}}(t-s ; \xi)\right)\right) d r\right) \\
\cdot\left|\operatorname{det} \partial_{\xi} \mathfrak{X}_{-1, \mathbf{g}}(t-s ; \xi)\right| d \xi d s \\
=\int_{0}^{t} \int_{\mathbb{R}^{N}} w\left(\mathfrak{X}_{-1, \mathbf{g}}(t-s ; \xi)\right) \cdot \varphi(\xi) \\
\cdot \exp \left(\int_{0}^{t-s}\left(u-\operatorname{div}_{\mathbf{x}} \mathbf{g}\right)\left(\mathfrak{X}_{-1, \mathbf{g}}(r ; \xi)\right) d r\right) d \xi d s \\
=\int_{\mathbb{R}^{N}} \varphi(\xi) \cdot \int_{0}^{t}\left(w\left(\mathfrak{X}_{-1, \mathbf{g}}(t-s ; \xi)\right)\right.
\end{aligned}
$$




$$
\left.\cdot \exp \left(\int_{0}^{t-s}\left(u-\operatorname{div}_{\mathbf{x}} \mathbf{g}\right)\left(\mathfrak{X}_{-1, \mathbf{g}}(r ; \xi)\right) d r\right)\right) d s d \xi
$$

Finally the claim results directly from Eq. (7).

Remark 25 The results of this subsection can be extended easily to nonautonomous linear problems as considered in Sect. 4.2, i.e., for any $T>0$ and coefficients $\widetilde{\mathbf{g}} \in L^{\infty}\left(0, T ; L^{\infty}\left(\mathbb{R}^{N}, \mathbb{R}^{N}\right)\right), \partial_{\mathbf{x}} \widetilde{\mathbf{g}} \in L^{\infty}\left(0, T ; L^{\infty}\left(\mathbb{R}^{N}, \mathbb{R}^{N \times N}\right)\right), \widetilde{u} \in$ $L^{\infty}\left(0, T ; L^{\infty}\left(\mathbb{R}^{N}\right)\right)$ and $\widetilde{w} \in L^{1}\left(0, T ; L^{p}\left(\mathbb{R}^{N}\right)\right)$.

According to Proposition 19, every initial function $f_{0} \in L^{p}\left(\mathbb{R}^{N}\right)$ leads to at most one weak solution $f \in L^{1}\left(0, T ; L^{p}\left(\mathbb{R}^{N}\right)\right)$ of the nonautonomous transport equation

$$
\partial_{t} f+\operatorname{div}_{\mathbf{x}}(f \widetilde{\mathbf{g}}(t))=\widetilde{u}(t) f+\widetilde{w}(t), \quad f(0)=f_{0} .
$$

Considering now the flow $\mathfrak{X}_{\alpha, \widetilde{\mathbf{g}}}:[0, T] \times \mathbb{R}^{N} \rightarrow \mathbb{R}^{N}$ of the nonautonomous differential equation

$$
\partial_{t} \mathfrak{X}_{\alpha, \widetilde{\mathbf{g}}}(t ; \mathbf{x})=\alpha \cdot \widetilde{\mathbf{g}}\left(t, \mathfrak{X}_{\alpha, \mathbf{g}}(t ; \mathbf{x})\right), \quad \mathfrak{X}_{\alpha, \widetilde{\mathbf{g}}}(0 ; \mathbf{x})=\mathbf{x}
$$

in the sense of Carathéodory solutions, we can follow the same arguments as in the proof of Proposition 20 for verifying that the function $\widetilde{f}:[0, T] \rightarrow L^{p}\left(\mathbb{R}^{N}\right)$ with

$$
\begin{aligned}
\int_{\mathbb{R}^{N}} \varphi \widetilde{f}(t) d \mathbf{x}= & \int_{\mathbb{R}^{N}} \varphi\left(\mathfrak{X}_{1, \widetilde{\mathbf{g}}}(t ; \mathbf{x})\right) \cdot \exp \left(\int_{0}^{t} \widetilde{u}\left(r, \mathfrak{X}_{1, \widetilde{\mathbf{g}}}(r ; \mathbf{x})\right) d r\right) f_{0} d \mathbf{x} \\
& +\int_{\mathbb{R}^{N}} \int_{0}^{t} \widetilde{w}(s, \mathbf{x}) \varphi\left(\mathfrak{X}_{1, \widetilde{\mathbf{g}}}(t-s ; \mathbf{x})\right) \\
& \cdot \exp \left(\int_{0}^{t-s} \widetilde{u}\left(r, \mathfrak{X}_{1, \widetilde{\mathbf{g}}}(r ; \mathbf{x})\right) d r\right) d s d \mathbf{x}
\end{aligned}
$$

for each $\varphi \in C_{c}^{1}\left(\mathbb{R}^{N}\right)$ is this unique weak solution of (15). This leads to the counterparts of the explicit representation in Proposition 23 and to the dominating function in Corollary 24 by using the area formula (e.g., [35, § 3.3, Theorem 2]) rather than the change of variables formula for Lebesgue integrals.

Finally, the weak solution $f:[0, T] \rightarrow L^{p}\left(\mathbb{R}^{N}\right)$ of the nonautonomous linear problem (15) satisfies

$$
\begin{aligned}
|f(t, \xi)| \leq e^{C \cdot t} \cdot\left(\left|f_{0}\left(\mathfrak{X}_{-1, \widetilde{\mathbf{g}}}(t ; \xi)\right)\right|+\int_{0}^{t}\left|w\left(s, \mathfrak{X}_{-1, \widetilde{\mathbf{g}}}(t-s ; \xi)\right)\right| d s\right) \\
|f(t, \xi)|^{p} \leq C e^{C \cdot t} \cdot\left(\left|f_{0}\left(\mathfrak{X}_{-1, \widetilde{\mathbf{g}}}(t ; \xi)\right)\right|^{p}+\int_{0}^{t}\left|w\left(s, \mathfrak{X}_{-1, \widetilde{\mathbf{g}}}(t-s ; \xi)\right)\right|^{p} d s\right)
\end{aligned}
$$

for Lebesgue-almost every $t \in[0, T]$ and Lebesgue-almost every $\xi \in \mathbb{R}^{N}$ with finite constants $C$ depending on $N, p, T,\left\|\partial_{\mathbf{x}} \widetilde{\mathbf{g}}\right\|_{L^{\infty}\left(0, T ; L^{\infty}\right)},\|\widetilde{u}\|_{L^{\infty}\left(0, T ; L^{\infty}\right)}$. 


\subsection{Autonomous linear problem with "more regular" coefficients: further estimates}

Definition 26 For any functions $\mathbf{g} \in C_{c}^{1}\left(\mathbb{R}^{N}, \mathbb{R}^{N}\right), u \in C^{1}\left(\mathbb{R}^{N}\right) \cap W^{1, \infty}\left(\mathbb{R}^{N}\right)$ and $w \in L^{p}\left(\mathbb{R}^{N}\right)$, let $\vartheta_{\mathbf{g}, u, w}^{f}:[0,1] \times L^{p}\left(\mathbb{R}^{N}\right) \rightarrow L^{p}\left(\mathbb{R}^{N}\right)$ be defined as the unique weak solution $\vartheta_{\mathbf{g}, u, w}^{f}\left(t, f_{0}\right):=f(t)$ of

$$
\partial_{t} f+\operatorname{div}_{\mathbf{x}}(f \mathbf{g})=u f+w \text { in }[0, t], \quad f(0)=f_{0},
$$

the existence and uniqueness of which are stated in Propositions 19 and 20 respectively.

Proposition 27 Suppose that $\mathbf{g}, \widehat{\mathbf{g}} \in C_{c}^{1}\left(\mathbb{R}^{N}, \mathbb{R}^{N}\right), u, \widehat{u} \in C^{1}\left(\mathbb{R}^{N}\right) \cap W^{1, \infty}\left(\mathbb{R}^{N}\right) \cap$ $L^{q}\left(\mathbb{R}^{N}\right)$ and $w, \widehat{w} \in L^{p}\left(\mathbb{R}^{N}\right)$. Then the following properties hold for any $f_{0}, f_{1} \in$ $L^{p}\left(\mathbb{R}^{N}\right), s, t \in[0,1]$ with $s+t \leq 1$ :

(1) $\vartheta_{\mathbf{g}, u, w}^{f}\left(0, f_{0}\right)=f_{0}$

(2) $\vartheta_{\mathbf{g}, u, w}^{f}\left(s+t, f_{0}\right)=\vartheta_{\mathbf{g}, u, w}^{f}\left(s, \vartheta_{\mathbf{g}, u, w}^{f}\left(t, f_{0}\right)\right)$

(3) $\left\|\vartheta_{\mathbf{g}, u, w}^{f}\left(t, f_{0}\right)\right\|_{L^{p}} \leq\left(\left\|f_{0}\right\|_{L^{p}}+\|w\|_{L^{p}} t\right) \cdot e^{\operatorname{const}(N) \cdot\left(1+\left\|\left[\operatorname{div}_{\mathbf{x}} \mathbf{g}\right]^{-}\right\|_{L^{\infty}}+\|u\|_{L^{\infty}}\right) \cdot t}$

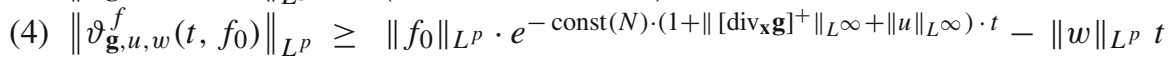

(5) $\breve{e}_{L^{p}}\left(f_{0}, \vartheta_{\mathbf{g}, u, w}^{f}\left(t, f_{0}\right)\right) \leq t \cdot \operatorname{const}\left(N,\|\mathbf{g}\|_{L^{\infty}},\left\|\left[\operatorname{div}_{\mathbf{x}} \mathbf{g}\right]^{-}\right\|_{L^{\infty}},\|u\|_{L^{\infty}}\right) \cdot\left(\left\|f_{0}\right\|_{L^{p}}\right.$ $\left.+\|w\|_{L^{p}}\right)$

(6) $e_{L^{p}}\left(f_{0}, \vartheta_{\mathbf{g}, u, w}^{f}\left(t, f_{0}\right)\right) \leq t \cdot \operatorname{const}\left(N,\|\mathbf{g}\|_{L^{\infty}},\left\|\operatorname{div}_{\mathbf{x}} \mathbf{g}\right\|_{L^{\infty}},\|u\|_{L^{\infty}}\right) \cdot\left(\left\|f_{0}\right\|_{L^{p}}\right.$ $\left.+\|w\|_{L^{p}}\right)$

(7) $d_{L^{p}}\left(\vartheta_{\mathbf{g}, u, w}^{f}\left(t, f_{0}\right), \quad \vartheta_{\mathbf{g}, u, w}^{f}\left(t, f_{1}\right)\right)$ $\leq \quad d_{L^{p}}\left(f_{0}, f_{1}\right)$ $e^{\text {const }(N)\left(1+\left\|\partial_{\mathbf{x}} \mathbf{g}\right\|_{L} \infty+\|u\|_{L} \infty+\left\|\nabla_{\mathbf{x}} u\right\|_{L} \infty\right) \cdot t}$

(8) $d_{L^{p}}\left(\vartheta_{\mathbf{g}, u, w}^{f}\left(t, f_{0}\right), \vartheta_{\widehat{\mathbf{g}}, \widehat{u}, \widehat{w}}^{f}\left(t, f_{0}\right)\right) \leq C \cdot\left(\left\|f_{0}\right\|_{L^{p}}+\|\widehat{w}\|_{L^{p}}\right) \cdot t\left(\|\mathbf{g}-\widehat{\mathbf{g}}\|_{L^{q}}+\right.$ $\left.\|u-\widehat{u}\|_{L^{q}}+\|w-\widehat{w}\|_{L^{p}}\right)$ with a constant $C=C\left(N, p,\left\|\partial_{\mathbf{x}} \mathbf{g}\right\|_{L^{\infty}},\left\|\partial_{\mathbf{x}} \widehat{\mathbf{g}}\right\|_{L^{\infty}}\right.$, $\left.\|u\|_{W^{1, \infty}},\|\widehat{u}\|_{W^{1, \infty}}\right)$.

Lemma 28 Under the assumptions of Proposition 20, let $\psi_{t, \varphi} \in C^{1}\left([0, t] \times \mathbb{R}^{N}\right)$ denote the unique solution to problem (11) for any $t \in[0, T]$ and $\varphi \in C_{c}^{1}\left(\mathbb{R}^{N}\right)$ given. In addition, let $\widehat{\psi}_{t, \varphi} \in C^{1}\left([0, t] \times \mathbb{R}^{N}\right)$ be the solution to problem (11) with instead the coefficients $\widehat{\mathbf{g}} \in C_{c}^{1}\left(\mathbb{R}^{N}, \mathbb{R}^{N}\right)$ and $\widehat{u} \in C^{1}\left(\mathbb{R}^{N}\right) \cap W^{1, \infty}\left(\mathbb{R}^{N}\right) \cap L^{q}\left(\mathbb{R}^{N}\right)$.

Then, the following a priori estimates

$$
\begin{aligned}
\left\|\nabla_{\mathbf{x}} \psi_{t, \varphi}(s ; \cdot)\right\|_{L^{q}} \leq & \left(\left\|\nabla_{\mathbf{x}} \varphi\right\|_{L^{q}}+\|\varphi\|_{L^{q}}(t-s)\left\|\nabla_{\mathbf{x}} u\right\|_{L^{\infty}}\right) \\
& \cdot e^{\operatorname{const}(N, p) \cdot\left(1+\left\|\partial_{\mathbf{x}} \mathbf{g}\right\|_{L^{\infty}}+\|u\|_{L^{\infty}}\right) \cdot t} \\
\left\|\psi_{t, \varphi}(s ; \cdot)-\widehat{\psi}_{t, \varphi}(s ; \cdot)\right\|_{L^{q}} \leq & \left(\|\varphi\|_{L^{\infty}}+\left\|\nabla_{\mathbf{x}} \varphi\right\|_{L^{\infty}}\right) \\
& \cdot C t e^{C t}\left(\|\mathbf{g}-\widehat{\mathbf{g}}\|_{L^{q}}+\|u-\widehat{u}\|_{L^{q}}\right)
\end{aligned}
$$

hold for every $0 \leq s \leq t \leq T$ and $\varphi \in C_{c}^{1}\left(\mathbb{R}^{N}\right)$ with a constant $C$ which depends on $N, p,\left\|\partial_{\mathbf{x}} \mathbf{g}\right\|_{L^{\infty}},\left\|\partial_{\mathbf{x}} \widehat{\mathbf{g}}\right\|_{L^{\infty}},\left\|\nabla_{\mathbf{x}} u\right\|_{L^{\infty}}$ and $\left\|\nabla_{\mathbf{x}} \widehat{u}\right\|_{L^{\infty}}$. 
Proof of Lemma 28 As before $\mathfrak{X}_{1, \mathbf{g}}$ denotes the solution of initial value problem (8). The explicit representation of $\psi_{t, \varphi}$, i.e., $\psi_{t, \varphi}(s ; \mathbf{x})=\varphi\left(\mathfrak{X}_{1, \mathbf{g}}(t-s ; \mathbf{x})\right)$. $e^{\int_{0}^{t-s} u\left(\mathfrak{X}_{1, \mathbf{g}}(r ; \mathbf{x})\right) d r}$, provides for the gradient with respect to $\mathbf{x}$

$$
\begin{aligned}
\left|\nabla_{\mathbf{x}} \psi_{t, \varphi}(s ; \cdot)\right| \leq & \left(\left|\nabla_{\mathbf{x}} \varphi\left(\mathfrak{X}_{1, \mathbf{g}}(t-s ; \cdot)\right) \cdot \partial_{\mathbf{x}} \mathfrak{X}_{1, \mathbf{g}}(t-s ; \cdot)\right|\right. \\
& \left.+\left|\varphi\left(\mathfrak{X}_{1, \mathbf{g}}(t-s ; \cdot)\right) \cdot \int_{0}^{t-s} \nabla_{\mathbf{x}} u\left(\mathfrak{X}_{1, \mathbf{g}}(r ; \cdot)\right) \cdot \partial_{\mathbf{x}} \mathfrak{X}_{1, \mathbf{g}}(r ; \cdot) d r\right|\right) \\
& \cdot e^{\int_{0}^{t-s} u\left(\mathfrak{X}_{1, \mathbf{g}}(r ; \cdot)\right) d r .}
\end{aligned}
$$

The change of variables formula for Lebesgue integrals leads to the following upper estimate of the first scalar product:

$$
\begin{aligned}
& \int_{\mathbb{R}^{N}}\left|\nabla_{\mathbf{x}} \varphi\left(\mathfrak{X}_{1, \mathbf{g}}(t-s ; \mathbf{x})\right) \cdot \partial_{\mathbf{x}} \mathfrak{X}_{1, \mathbf{g}}(t-s ; \mathbf{x})\right|^{q} d \mathbf{x} \\
& \quad \leq\left\|\partial_{\mathbf{x}} \mathfrak{X}_{1, \mathbf{g}}(t-s ; \cdot)\right\|_{L^{\infty}}^{q} \cdot \int_{\mathbb{R}^{N}}\left|\nabla_{\mathbf{x}} \varphi\left(\mathfrak{X}_{1, \mathbf{g}}(t-s ; \mathbf{x})\right)\right|^{q} d \mathbf{x} \\
& \quad \stackrel{(9)}{\leq} e^{\operatorname{const}(N, p) \cdot\left(1+\left\|\partial_{\mathbf{x}} \mathbf{g}\right\|_{L^{\infty}}\right) \cdot t} \cdot\left\|\operatorname{det}\left(\partial_{\mathbf{x}} \mathfrak{X}_{1, \mathbf{g}}(t ; \cdot)\right)^{-1}\right\|_{L^{\infty}}\left\|\nabla_{\mathbf{x}} \varphi\right\|_{L^{q}}^{q} \\
& \quad \stackrel{(10)}{\leq} e^{\operatorname{const}(N, p) \cdot\left(1+\left\|\partial_{\mathbf{x}} \mathbf{g}\right\|_{L^{\infty}}\right) \cdot t} \cdot\left\|\nabla_{\mathbf{x}} \varphi\right\|_{L^{q}}^{q} .
\end{aligned}
$$

The corresponding conclusion for the second term and Minkowski inequality imply

$$
\begin{aligned}
\left\|\nabla_{\mathbf{x}} \psi_{t, \varphi}(s ; \cdot)\right\|_{L^{q}} \leq & e^{\operatorname{const}(N, p) \cdot\left(1+\left\|\partial_{\mathbf{x}} \mathbf{g}\right\|_{L^{\infty}}+\|u\|_{L^{\infty}}\right) \cdot t} \\
& \cdot\left(\left\|\nabla_{\mathbf{x}} \varphi\right\|_{L^{q}}+\|\varphi\|_{L^{q}}(t-s)\left\|\nabla_{\mathbf{x}} u\right\|_{L^{\infty}}\right) .
\end{aligned}
$$

To "compare" the solutions $\psi_{t, \varphi}$ and $\widehat{\psi}_{t, \varphi}$ related to the coefficients $\mathbf{g}, u$ and $\widehat{\mathbf{g}}, \widehat{u}$, respectively, we use the auxiliary functions $\mathbf{g}_{\lambda}:=\lambda \cdot \mathbf{g}+(1-\lambda) \cdot \widehat{\mathbf{g}}, u_{\lambda}:=\lambda \cdot u+(1-\lambda) \cdot \widehat{u}$ and $\Psi_{t, \varphi}^{\lambda} \in C^{1}\left([0, t] \times \mathbb{R}^{N}\right)$, where $0 \leq \lambda \leq 1$, with

$$
\Psi_{t, \varphi}^{\lambda}(s ; \cdot):=\varphi\left(\mathfrak{X}_{1, g_{\lambda}}(t-s ; \cdot)\right) \cdot \exp \left(\int_{0}^{t-s} u_{\lambda}\left(\mathfrak{X}_{1, g_{\lambda}}(r ; \cdot)\right) d r\right) .
$$

In particular, $\Psi_{t, \varphi}^{1}=\psi_{t, \varphi}$ and $\Psi_{t, \varphi}^{0}=\widehat{\psi}_{t, \varphi}$. Furthermore, the integral form of differential equation (8), i.e.,

$$
\mathfrak{X}_{1, \mathbf{g}_{\lambda}}(t ; \mathbf{x})=\mathbf{x}+\int_{0}^{t} \mathbf{g}_{\lambda}\left(\mathfrak{X}_{1, \mathbf{g}_{\lambda}}(s ; \mathbf{x})\right) d s
$$

provides an integral equation for the partial derivative $\mathfrak{X}_{1, \mathbf{g}_{\lambda}}(\cdot ; \mathbf{x})$ w.r.t. parameter $\lambda$ :

$$
\partial_{\lambda} \mathfrak{X}_{1, \mathbf{g} \lambda}(t ; \mathbf{x})=\int_{0}^{t}\left((\mathbf{g}-\widehat{\mathbf{g}})\left(\mathfrak{X}_{1, \mathbf{g}_{\lambda}}(s ; \mathbf{x})\right)+\partial_{\mathbf{x}} \mathbf{g}_{\lambda}\left(\mathfrak{X}_{1, \mathbf{g}_{\lambda}}(s ; \mathbf{x})\right) \cdot \partial_{\lambda} \mathfrak{X}_{1, \mathbf{g}_{\lambda}}(s ; \mathbf{x})\right) d s
$$




$$
\begin{aligned}
\left|\partial_{\lambda} \mathfrak{X}_{1, \mathbf{g}_{\lambda}}(t ; \mathbf{x})\right| \leq & 2 \int_{0}^{t} \max \left\{\left.|(\mathbf{g}-\widehat{\mathbf{g}})|_{\mathfrak{X}_{1, \mathbf{g}_{\lambda}}(s ; \mathbf{x})}|,| \partial_{\mathbf{x}} \mathbf{g}_{\lambda}\right|_{\mathfrak{X}_{1, \mathbf{g} \lambda}(s ; \mathbf{x})} \cdot \partial_{\lambda} \mathfrak{X}_{1, \mathbf{g}_{\lambda}}(s ; \mathbf{x}) \mid\right\} d s \\
\leq & 2 t^{\frac{1}{p}}\left(\int _ { 0 } ^ { t } \operatorname { m a x } \left\{\left.|(\mathbf{g}-\widehat{\mathbf{g}})|_{\mathfrak{X}_{1, \mathbf{g}}(s ; \mathbf{x})}\right|^{q},\right.\right. \\
& \left.\left.\left.\left|\partial_{\mathbf{x}} \mathbf{g}_{\lambda}\right|_{\mathfrak{X}_{1, \mathbf{g}_{\lambda}}(s ; \mathbf{x})} \cdot \partial_{\lambda} \mathfrak{X}_{1, \mathbf{g}_{\lambda}}(s ; \mathbf{x})\right|^{q}\right\} d s\right)^{\frac{1}{q}} \\
\left|\partial_{\lambda} \mathfrak{X}_{1, \mathbf{g}_{\lambda}}(t ; \mathbf{x})\right|^{q} \leq & 2^{q} t^{\frac{q}{p}} \int_{0}^{t} \max \left\{|(\mathbf{g}-\widehat{\mathbf{g}})|_{\left.\mathfrak{X}_{1, \mathbf{g}}(s ; \mathbf{x})\right|^{q}}\right. \\
& \left.\left.\left|\partial_{\mathbf{x}} \mathbf{g}_{\lambda}\right|_{\mathfrak{X}_{1, \mathbf{g}_{\lambda}}(s ; \mathbf{x})} \cdot \partial_{\lambda} \mathfrak{X}_{1, \mathbf{g}_{\lambda}}(s ; \mathbf{x})\right|^{q}\right\} d s \\
\leq & 2^{q} t^{\frac{q}{p}} \int_{0}^{t}\left(\left.|(\mathbf{g}-\widehat{\mathbf{g}})|_{\mathfrak{X}_{1, \mathbf{g}_{\lambda}}(s ; \mathbf{x})}\right|^{q}\right. \\
& \left.+\left.\left|\partial_{\mathbf{x}} \mathbf{g}_{\lambda}\right|_{\mathfrak{X}_{1, \mathbf{g}}(s ; \mathbf{x})} \cdot \partial_{\lambda} \mathfrak{X}_{1, \mathbf{g}_{\lambda}}(s ; \mathbf{x})\right|^{q}\right) d s
\end{aligned}
$$

so

$\left\|\partial_{\lambda} \mathfrak{X}_{1, \mathbf{g}_{\lambda}}(t ; \cdot)\right\|_{L^{q}}^{q} \leq 2^{q} t^{\frac{q}{p}} \int_{0}^{t}\left(\|\mathbf{g}-\widehat{\mathbf{g}}\|_{L^{q}}^{q} \cdot e^{c s}+\left\|\partial_{\mathbf{x}} \mathbf{g}_{\lambda}\right\|_{L^{\infty}}^{q}\left\|\partial_{\lambda} \mathfrak{X}_{1, \mathbf{g} \lambda}(s ; \mathbf{x})\right\|_{L^{q}}^{q}\right) d s$ with a constant $c=c\left(N,\left\|\left[\operatorname{div}_{\mathbf{x}} \mathbf{g}\right]^{-}\right\|_{L^{\infty}},\left\|\left[\operatorname{div}_{\mathbf{x}} \widehat{\mathbf{g}}\right]^{-}\right\|_{L^{\infty}}\right)$. Hence by the Gronwall's inequality,

$$
\left\|\partial_{\lambda} \mathfrak{X}_{1, g_{\lambda}}(t ; \cdot)\right\|_{L^{q}} \leq\|\mathbf{g}-\widehat{\mathbf{g}}\|_{L^{q}} \cdot C t e^{C t}
$$

with some constant $C=C\left(N, p,\left\|\partial_{\mathbf{x}} \mathbf{g}\right\|_{L^{\infty}},\left\|\partial_{\mathbf{x}} \widehat{\mathbf{g}}\right\|_{L^{\infty}}\right)$. Thus,

$$
\begin{aligned}
\partial_{\lambda} \Psi_{t, \varphi}^{\lambda}(s ; \mathbf{x})= & e^{\int_{0}^{t-s} u_{\lambda}\left(\mathfrak{X}_{1, g_{\lambda}}(r ; \mathbf{x})\right) d r} \cdot\left\{\left.\partial_{\mathbf{x}} \varphi\right|_{\mathfrak{X}_{1, g_{\lambda}}(t-s ; \mathbf{x})} \cdot \partial_{\lambda} \mathfrak{X}_{1, g_{\lambda}}(t-s ; \mathbf{x})\right. \\
& +\left.\varphi\right|_{\mathfrak{X}_{1, g_{\lambda}}(t-s ; \mathbf{x})} \cdot \int_{0}^{t-s}\left(\left.(u-\widehat{u})\right|_{\mathfrak{X}_{1, g_{\lambda}}(r ; \mathbf{x})}\right. \\
& \left.\left.+\left.\partial_{\mathbf{x}} u_{\lambda}\right|_{\mathfrak{X}_{1, g_{\lambda}}(r ; \mathbf{x})} \cdot \partial_{\lambda} \mathfrak{X}_{1, g_{\lambda}}(r ; \mathbf{x})\right) d r\right\}
\end{aligned}
$$

implies the upper estimate

$$
\begin{aligned}
\left\|\partial_{\lambda} \Psi_{t, \varphi}^{\lambda}(s ; \cdot)\right\|_{L^{q}} \leq & e^{(t-s) \cdot\left(\|u\|_{L^{\infty}}+\|\widehat{u}\|_{L^{\infty}}\right)} \\
& \cdot\left\{\left\|\partial_{\mathbf{x}} \varphi\left(\mathfrak{X}_{1, g_{\lambda}}(t-s ; \cdot)\right)\right\|_{L^{\infty}} \cdot\left\|\partial_{\lambda} \mathfrak{X}_{1, g_{\lambda}}(t-s ; \cdot)\right\|_{L^{q}}\right. \\
& +\|\varphi\|_{L^{\infty}} \cdot(t-s) \\
& \left.\times\left(\|u-\widehat{u}\|_{L^{q}} \cdot e^{c(t-s)}+\left\|\partial_{\mathbf{x}} u_{\lambda}\right\|_{L^{\infty}} \cdot \sup _{r \leq t-s}\left\|\partial_{\lambda} \mathfrak{X}_{1, g_{\lambda}}(r ; \cdot)\right\|_{L^{q}}\right)\right\} \\
\leq & C e^{C t} \cdot(t-s) \cdot\left\{\left\|\nabla_{\mathbf{x}} \varphi\right\|_{L^{\infty}}\|\mathbf{g}-\widehat{\mathbf{g}}\|_{L^{q}}\right. \\
& +\|\varphi\|_{L^{\infty}}\left(\|u-\widehat{u}\|_{L^{q}}+\left(\left\|\partial_{\mathbf{x}} u\right\|_{L^{\infty}}+\left\|\partial_{\mathbf{x}} \widehat{u}\right\|_{L^{\infty}}\right)\right. \\
& \left.\left.\cdot\|\mathbf{g}-\widehat{\mathbf{g}}\|_{L^{q}}(t-s)\right)\right\}
\end{aligned}
$$


with some constant $C=C\left(N, p,\left\|\partial_{\mathbf{x}} \mathbf{g}\right\|_{L^{\infty}},\left\|\partial_{\mathbf{x}} \widehat{\mathbf{g}}\right\|_{L^{\infty}},\|u\|_{L^{\infty}},\|\widehat{u}\|_{L^{\infty}}\right)<\infty$. The last claimed inequality now results from

$$
\left\|\psi_{t, \varphi}(s ; \cdot)-\widehat{\psi}_{t, \varphi}(s ; \cdot)\right\|_{L^{q}} \leq \int_{0}^{1}\left\|\partial_{\lambda} \Psi_{t, \varphi}^{\lambda}(s ; \cdot)\right\|_{L^{q}} d \lambda
$$

Proof of Proposition 27 The statements of the theorem will be considered sequentially.

(1) The property $\vartheta_{\mathbf{g}, u, w}^{f}\left(0, f_{0}\right)=f_{0}$ results immediately from Definition 26 of $\vartheta_{\mathbf{g}, u, w}^{f}$.

(2) The semi-group property $\vartheta_{\mathbf{g}, u, w}^{f}\left(s+t, f_{0}\right)=\vartheta_{\mathbf{g}, u, w}^{f}\left(s, \vartheta_{\mathbf{g}, u, w}^{f}\left(t, f_{0}\right)\right)$ is a consequence of the uniqueness of solutions to the initial value problem (5) as stated in Proposition 19.

(3) This upper $L^{p}$ bound has already been formulated (and proved) in Proposition 20.

(4) Choose any $t \in[0,1]$ and $f_{0} \in L^{p}\left(\mathbb{R}^{N}\right)$. The characterizing condition (6) on weak solutions implies immediately that $f:[0, t] \rightarrow L^{p}\left(\mathbb{R}^{N}\right)$ is a weak solution of initial value problem (5) if and only if $\widehat{f}:=f(t-\cdot):[0, t] \rightarrow L^{p}\left(\mathbb{R}^{N}\right)$ is a weak solution of

$$
\partial_{t} \widehat{f}-\operatorname{div}_{\mathbf{x}}(\widehat{f} \mathbf{g})=-u \widehat{f}-w \text { in }[0, t], \quad \widehat{f}(0)=f(t)
$$

From the uniqueness mentioned in Proposition 19, we conclude that the weak solutions of (17) characterize the inverse of $\vartheta_{\mathbf{g}, u, w}^{f}(t, \cdot): L^{p}\left(\mathbb{R}^{N}\right) \rightarrow L^{p}\left(\mathbb{R}^{N}\right)$, i.e.,

$$
\vartheta_{\mathbf{g}, u, w}^{f}(t, \cdot)^{-1}: L^{p}\left(\mathbb{R}^{N}\right) \rightarrow L^{p}\left(\mathbb{R}^{N}\right), \quad f(t) \mapsto \widehat{f}(t)=f_{0},
$$

where $\vartheta_{\mathbf{g}, u, w}^{f}\left(t, f_{0}\right)=f(t)$. Now the lower $L^{p}$ bound of $f(t):=\vartheta_{\mathbf{g}, u, w}^{f}\left(t, f_{0}\right)$ results from the counterpart of property (3) for Eq. (17):

$$
\left\|f_{0}\right\|_{L^{p}} \leq\left(\|f(t)\|_{L^{p}}+\|w\|_{L^{p}} t\right) \cdot e^{\operatorname{const}(N, p) \cdot\left(1+\left\|\left[\operatorname{div}_{\mathbf{X}}(-\mathbf{g})\right]^{-}\right\|_{\left.L^{\infty}+\|u\|_{L^{\infty}}\right) \cdot t} .\right.}
$$

(5) For $f(t):=\vartheta_{\mathbf{g}, u, w}^{f}\left(t, f_{0}\right), t \in[0,1]$ and $\varphi \in C_{c}^{1}\left(\mathbb{R}^{N}\right)$, Eq. (6) and property (3) imply

$$
\begin{aligned}
\int_{\mathbb{R}^{N}} \varphi\left(f(t)-f_{0}\right) d \mathbf{x}= & \int_{0}^{t} \int_{\mathbb{R}^{N}}\left(f(s, \cdot)\left(\mathbf{g} \cdot \nabla_{\mathbf{x}} \varphi+u \varphi\right)\right) d \mathbf{x} d s \\
& +t \int_{\mathbb{R}^{N}} \varphi w d \mathbf{x} \\
\leq & \int_{0}^{t}\left(\| f ( s ) \| _ { L ^ { p } } \left(\|\mathbf{g}\|_{L^{\infty}}\|\nabla \varphi\|_{L^{q}}\right.\right. \\
& \left.\left.+\|u\|_{L^{\infty}}\|\varphi\|_{L^{q}}\right)\right) d s+t \cdot\|\varphi\|_{L^{q}}\|w\|_{L^{p}} \\
\leq & t \cdot\left(\|\varphi\|_{L^{q}}+\|\nabla \varphi\|_{L^{q}}\right) \cdot\left(\left\|f_{0}\right\|_{L^{p}}+\|w\|_{L^{p}}\right)
\end{aligned}
$$




$$
\begin{aligned}
& \left(1+\|\mathbf{g}\|_{L^{\infty}}+\|u\|_{L^{\infty}}\right) \\
& \cdot \exp \left(\operatorname{const}(N, p) \cdot\left(1+\left\|\left[\operatorname{div}_{\mathbf{x}} \mathbf{g}\right]^{-}\right\|_{L^{\infty}}+\|u\|_{L^{\infty}}\right)\right) .
\end{aligned}
$$

The supremum w.r.t. all $\varphi \in C_{c}^{1}\left(\mathbb{R}^{N}\right)$ satisfying both $\|\varphi\|_{W^{1, q}} \leq 1$ and $\|\varphi\|_{W^{1, \infty}} \leq$ 1 leads to the claimed estimate of $\breve{e}_{L^{p}}\left(f_{0}, f(t)\right)$.

(6) Statements (3) and (4) imply the right continuity of $[0,1] \rightarrow \mathbb{R}, t \mapsto$ $\left\|\vartheta_{\mathbf{g}, u, w}^{f}\left(t, f_{0}\right)\right\|_{L^{p}}$ at $t=0$. Due to the semi-group property (2), the norm is right continuous at every $t \in[0,1)$. By means of (18), we similarly obtain the left continuity of $\left\|\vartheta_{\mathbf{g}, u, w}^{f}\left(\cdot, f_{0}\right)\right\|_{L^{p}}$ in $(0,1]$.

Furthermore, statements (3) and (4) guarantee that the right Dini derivative is bounded at each time instant $t \in[0,1)$ :

$$
\begin{aligned}
& \underset{h \downarrow 0}{\limsup }\left|\frac{\left\|\vartheta_{\mathbf{g}, u, w}^{f}\left(t+h, f_{0}\right)\right\|_{L^{p}}-\left\|\vartheta_{\mathbf{g}, u, w}^{f}\left(t, f_{0}\right)\right\|_{L^{p}}}{h}\right| \\
& \quad \leq \operatorname{const}\left(N, p,\left\|\operatorname{div}_{\mathbf{x}} \mathbf{g}\right\|_{L^{\infty}},\|u\|_{L^{\infty}}\right) \cdot\left(\left\|\vartheta_{\mathbf{g}, u, w}^{f}\left(t, f_{0}\right)\right\|_{L^{p}}+\|w\|_{L^{p}}\right) \\
& \stackrel{(3)}{\leq} \operatorname{const}\left(N, p,\left\|\operatorname{div}_{\mathbf{x}} \mathbf{g}\right\|_{L^{\infty}},\|u\|_{L^{\infty}}\right) \cdot\left(\left\|f_{0}\right\|_{L^{p}}+\|w\|_{L^{p}}\right),
\end{aligned}
$$

so the function $\left\|\vartheta_{\mathbf{g}, u, w}^{f}\left(\cdot, f_{0}\right)\right\|_{L^{p}}:[0,1] \rightarrow[0, \infty)$ is even Lipschitz continuous. Together with property (5), this leads to the claimed Lipschitz continuity of $\vartheta_{\mathbf{g}, u, w}^{f}\left(\cdot, f_{0}\right)$ w.r.t. the metric $e_{L^{p}}$.

(7) For every test function $\varphi \in C_{c}^{1}\left(\mathbb{R}^{N}\right)$, the representations (7), (12) guarantee for the solutions $\vartheta_{\mathbf{g}, u, w}^{f}\left(t, f_{0}\right)$ and $\vartheta_{\mathbf{g}, u, w}^{f}\left(t, f_{1}\right)$ related to initial states $f_{0}, f_{1} \in L^{p}\left(\mathbb{R}^{N}\right)$, respectively,

$$
\begin{gathered}
\int_{\mathbb{R}^{N}} \varphi \cdot\left(\vartheta_{\mathbf{g}, u, w}^{f}\left(t, f_{0}\right)-\vartheta_{\mathbf{g}, u, w}^{f}\left(t, f_{1}\right)\right) d \mathbf{x}=\int_{\mathbb{R}^{N}} \psi_{t, \varphi}(0 ; \mathbf{x})\left(f_{0}-f_{1}\right)(\mathbf{x}) d \mathbf{x} \\
=\int_{\mathbb{R}^{N}} \varphi\left(\mathfrak{X}_{1, \mathbf{g}}(t ; \mathbf{x})\right) \cdot \exp \left(\int_{0}^{t} u\left(\mathfrak{X}_{1, \mathbf{g}}(r ; \mathbf{x})\right) d r\right)\left(f_{0}-f_{1}\right)(\mathbf{x}) d \mathbf{x}
\end{gathered}
$$

By means of density arguments, this relation can be extended to any auxiliary function $\varphi \in C_{c}^{0}\left(\mathbb{R}^{N}\right) \cap W^{1, \infty}\left(\mathbb{R}^{N}\right)$. Due to the assumptions $\mathbf{g} \in C_{c}^{1}\left(\mathbb{R}^{N}, \mathbb{R}^{N}\right), u \in C^{1}\left(\mathbb{R}^{N}\right) \cap$ $W^{1, \infty}\left(\mathbb{R}^{N}\right)$, the function from $\mathbb{R}^{N}$ to $\mathbb{R}^{N}$ defined by

$$
\mathbf{x} \mapsto \varphi\left(\mathfrak{X}_{1, \mathbf{g}}(t ; \mathbf{x})\right) \cdot \exp \left(\int_{0}^{t} u\left(\mathfrak{X}_{1, \mathbf{g}}(r ; \mathbf{x})\right) d r\right)
$$

is Lipschitz continuous with compact support in $\mathbb{R}^{N}$ at each time instant $t \in[0,1]$ and, the following a priori estimates hold

$$
\begin{aligned}
&\left\|\varphi\left(\mathfrak{X}_{1, \mathbf{g}}(t ; \cdot)\right)\right\|_{L^{q}}^{q} \leq\|\varphi\|_{L^{q}}^{q} \cdot\left\|\operatorname{det}\left(\partial_{\mathbf{x}} \mathfrak{X}_{1, \mathbf{g}}(t ; \cdot)\right)^{-1}\right\|_{L^{\infty}} \\
& \stackrel{(10)}{\leq}\|\varphi\|_{L^{q}}^{q} \cdot e^{\operatorname{const}(N) \cdot\left\|\left[\operatorname{div}_{\mathbf{x}} \mathbf{g}\right]^{-}\right\|_{L^{\infty} \cdot t}},
\end{aligned}
$$




$$
\begin{aligned}
& \left\|\partial_{\mathbf{x}}\left(\varphi \circ \mathfrak{X}_{1, \mathbf{g}}(t ; \cdot)\right)\right\|_{L^{\infty}} \leq\left\|\nabla_{\mathbf{x}} \varphi\right\|_{L^{\infty}} \cdot\left\|\partial_{\mathbf{x}} \mathfrak{X}_{1, \mathbf{g}}(t ; \cdot)\right\|_{L^{\infty}} \\
& \stackrel{(9)}{\leq}\left\|\nabla_{\mathbf{x}} \varphi\right\|_{L^{\infty}} \cdot e^{\operatorname{const}(N) \cdot\left(1+\left\|\partial_{\mathbf{x}} \mathbf{g}\right\|_{L} \infty\right) \cdot t} \text {. }
\end{aligned}
$$

Correspondingly, the second factor in representation (19) of the difference, i.e., the expression $\eta_{\mathbf{g}, u, t}: \mathbb{R}^{N} \rightarrow \mathbb{R}$ defined by

$$
\mathbf{x} \mapsto \exp \left(\int_{0}^{t} u\left(\mathfrak{X}_{1, \mathbf{g}}(r ; \mathbf{x})\right) d r\right)
$$

and its gradient are essentially bounded with the a priori bound

$$
\begin{aligned}
\left\|\nabla_{\mathbf{x}} \eta_{\mathbf{g}, u, t}\right\|_{L^{\infty}} & \leq e^{\|u\|_{L^{\infty} t}} \cdot \int_{0}^{t}\left\|\nabla_{\mathbf{x}} u\left(\mathfrak{X}_{1, \mathbf{g}}(r ; \cdot)\right)\right\|_{L^{\infty}}\left\|\partial_{\mathbf{x}} \mathfrak{X}_{1, \mathbf{g}}(r ; \cdot)\right\|_{L^{\infty}} d r \\
& \stackrel{(9)}{\leq} e^{\operatorname{const}(N)\left(1+\left\|\partial_{\mathbf{x}} \mathbf{g}\right\|_{L^{\infty}}+\|u\|_{\left.L^{\infty}\right) t}\right.} \cdot\left\|\nabla_{\mathbf{x}} u\right\|_{L^{\infty}} t .
\end{aligned}
$$

Hence, standard arguments (about homogeneity w.r.t. the norm of test functions) lead to

$$
\begin{aligned}
& d_{L^{p}}\left(\vartheta_{\mathbf{g}, u, w}^{f}\left(t, f_{0}\right), \vartheta_{\mathbf{g}, u, w}^{f}\left(t, f_{1}\right)\right) \\
& \stackrel{(19)}{=} \sup \left\{\int_{\mathbb{R}^{N}} \varphi\left(\mathfrak{X}_{1, \mathbf{g}}(t ; \cdot)\right) \eta_{\mathbf{g}, u, t} \cdot\left(f_{0}-f_{1}\right) d \mathbf{x} \mid\right. \\
& \left.\varphi \in C_{c}^{1}\left(\mathbb{R}^{N}\right),\|\varphi\|_{L^{q}} \leq 1,\|\varphi\|_{L^{\infty}} \leq 1,\left\|\nabla_{\mathbf{x}} \varphi\right\|_{L^{\infty}} \leq 1\right\} \\
& \leq e^{\operatorname{const}(N)\left(1+\left\|\partial_{\mathbf{x}} \mathbf{g}\right\|_{L^{\infty}}+\|u\|_{L^{\infty}}+\left\|\nabla_{\mathbf{x}} u\right\|_{L^{\infty}}\right) \cdot t} \cdot d_{L^{p}}\left(f_{0}, f_{1}\right) .
\end{aligned}
$$

(8) For every test function $\varphi \in C_{c}^{1}\left(\mathbb{R}^{N}\right)$, let $\psi_{t, \varphi}, \widehat{\psi}_{t, \varphi} \in C^{1}\left([0, t] \times \mathbb{R}^{N}\right)$ denote the auxiliary functions as specified in Lemma 28 . We conclude from the estimates in Lemmas 22, 28

$$
\begin{aligned}
& \int_{\mathbb{R}^{N}} \varphi \cdot\left(\vartheta_{\mathbf{g}, u, w}^{f}\left(t, f_{0}\right)-\vartheta_{\widehat{\mathbf{g}}, \widehat{u}, \widehat{w}}^{f}\left(t, f_{0}\right)\right) d \mathbf{x} \\
& \stackrel{(7)}{=} \int_{\mathbb{R}^{N}}\left(\left(\psi_{t, \varphi}(0 ; \cdot)-\widehat{\psi}_{t, \varphi}(0 ; \cdot)\right) f_{0}\right. \\
& \left.\quad+w \int_{0}^{t} \psi_{t, \varphi}(s ; \cdot) d s-\widehat{w} \int_{0}^{t} \widehat{\psi}_{t, \varphi}(s ; \cdot) d s\right) d \mathbf{x} \\
& \leq\left\|\psi_{t, \varphi}(0 ; \cdot)-\widehat{\psi}_{t, \varphi}(0 ; \cdot)\right\|_{L^{q}}\left\|f_{0}\right\|_{L^{p}}+\|w-\widehat{w}\|_{L^{p}} \cdot \sup _{s \leq t}\left\|\psi_{t, \varphi}(s ; \cdot)\right\|_{L^{q}} t \\
& \quad+\|\widehat{w}\|_{L^{p}} \cdot \sup _{s \leq t}\left\|\psi_{t, \varphi}(s ; \cdot)-\widehat{\psi}_{t, \varphi}(s ; \cdot)\right\|_{L^{p}} t \\
& \leq\left(\|\varphi\|_{L^{\infty}}+\left\|\nabla_{\mathbf{x}} \varphi\right\|_{L^{\infty}}\right)\left(\left\|f_{0}\right\|_{L^{p}}+\|\widehat{w}\|_{L^{p}}\right) \cdot C e^{C t} t\left(\|\mathbf{g}-\widehat{\mathbf{g}}\|_{L^{q}}+\|u-\widehat{u}\|_{L^{q}}\right) \\
& \quad+\|\varphi\|_{L^{q}} \cdot C e^{C t} t\|w-\widehat{w}\|_{L^{p}}
\end{aligned}
$$




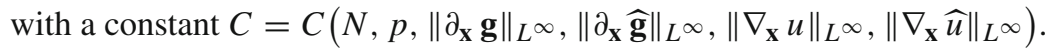

\subsection{Extending existence and estimates for autonomous linear problems to "less regular" coefficients}

In comparison with Proposition 20 about existence of solutions, the coefficients $\mathbf{g}, u, w$ are now supposed to satisfy the weaker regularity assumptions of the main Theorem 4, but we still consider the autonomous problem:

Proposition 29 Assume $\mathbf{g} \in L^{\infty}\left(\mathbb{R}^{N}, \mathbb{R}^{N}\right) \cap L^{q}, \partial_{\mathbf{x}} \mathbf{g} \in L^{\infty}\left(\mathbb{R}^{N}, \mathbb{R}^{N \times N}\right), u \in$ $L^{\infty}\left(\mathbb{R}^{N}\right) \cap L^{q}, \nabla_{\mathbf{x}} u \in L^{\infty}\left(\mathbb{R}^{N}, \mathbb{R}^{N}\right), w \in L^{p}\left(\mathbb{R}^{N}\right)$. Then for any initial function $f_{0} \in L^{p}\left(\mathbb{R}^{N}\right)$, there exists a weak solution $f:[0,1] \rightarrow L^{p}\left(\mathbb{R}^{N}\right)$ of the following initial value problem in the sense of Proposition 20

$$
\partial_{t} f+\operatorname{div}_{\mathbf{x}}(f \mathbf{g})=u f+w \text { in }[0,1], \quad f(0)=f_{0} .
$$

Furthermore, this solution is unique and denoted by $\vartheta_{\mathbf{g}, u, w}^{f}\left(\cdot, f_{0}\right):[0,1] \rightarrow L^{p}\left(\mathbb{R}^{N}\right)$. Finally, all estimates in Proposition 27 hold under these weaker regularity assumptions.

The proof is essentially based on smoothing the coefficients $\mathbf{g}, u$ such that Proposition 20 can be applied without any significant effects on the a priori estimates.

The following lemma can be verified quite easily by means of mollifying with some Dirac sequence of smooth functions (see, e.g., [47, Section VIII.3]) in combination with any cut-off function for the compact support. It is worth mentioning that this lemma states just the $L^{q}$ convergence of the sequence $\left(\mathbf{g}_{k}\right)_{k \in \mathbb{N}}$, but not the $L^{\infty}$ convergence of the derivatives $\left(\partial_{\mathbf{x}} \mathbf{g}_{k}\right)_{k \in \mathbb{N}}$.

Lemma $30 C^{\infty}\left(\mathbb{R}^{N}, \mathbb{R}^{m}\right) \cap L^{q}\left(\mathbb{R}^{N}, \mathbb{R}^{m}\right)$ is dense in $\left\{\mathbf{h} \in L^{q}\left(\mathbb{R}^{N}, \mathbb{R}^{m}\right) \mid \partial_{\mathbf{x}} \mathbf{h} \in L^{\infty}\right.$ $\left.\left(\mathbb{R}^{N}, \mathbb{R}^{m \times N}\right)\right\}$ w.r.t. the $L^{q}$ norm. In particular, for every function $\mathbf{h} \in L^{q}\left(\mathbb{R}^{N}, \mathbb{R}^{m}\right)$ with distributional derivative $\partial_{\mathbf{x}} \mathbf{h} \in L^{\infty}\left(\mathbb{R}^{N}, \mathbb{R}^{m \times N}\right)$, there exists a sequence $\left(\mathbf{h}_{k}\right)_{k \in \mathbb{N}}$ in $C^{\infty}\left(\mathbb{R}^{N}, \mathbb{R}^{m}\right)$ with

$$
\lim _{k \rightarrow \infty}\left\|\mathbf{h}_{k}-\mathbf{h}\right\|_{L^{q}}=0, \quad \sup _{k \in \mathbb{N}}\left\|\mathbf{h}_{k}\right\|_{L^{q}} \leq\|\mathbf{h}\|_{L^{q}}, \quad \sup _{k \in \mathbb{N}}\left\|\partial_{\mathbf{x}} \mathbf{h}_{k}\right\|_{L^{\infty}} \leq\left\|\partial_{\mathbf{x}} \mathbf{h}\right\|_{L^{\infty}}
$$

and (if $m=N$ ) $\sup _{k \in \mathbb{N}}\left\|\operatorname{div}_{\mathbf{x}} \mathbf{h}_{k}\right\|_{L^{\infty}} \leq\left\|\operatorname{div}_{\mathbf{x}} \mathbf{h}\right\|_{L^{\infty}}$.

Moreover, for any function $\mathbf{h} \in L^{\infty}\left(\mathbb{R}^{N}, \mathbb{R}^{m}\right) \cap L^{q}\left(\mathbb{R}^{N}, \mathbb{R}^{m}\right)$ with $\partial_{\mathbf{x}} \mathbf{h} \in$ $L^{\infty}\left(\mathbb{R}^{N}, \mathbb{R}^{m \times N}\right)$, the sequence $\left(\mathbf{h}_{k}\right)_{k \in \mathbb{N}}$ in $C_{c}^{\infty}\left(\mathbb{R}^{N}, \mathbb{R}^{m}\right)$ can be constructed in such a way that $\lim _{k \rightarrow \infty}\left\|\mathbf{h}_{k}-\mathbf{h}\right\|_{L^{q}}=0$ in addition to

$\sup _{k \in \mathbb{N}}\left\|\mathbf{h}_{k}\right\|_{L^{q}} \leq\|\mathbf{h}\|_{L^{q}}, \quad \sup _{k \in \mathbb{N}}\left\|\mathbf{h}_{k}\right\|_{L^{\infty}} \leq\|\mathbf{h}\|_{L^{\infty}}, \quad \sup _{k \in \mathbb{N}}\left\|\partial_{\mathbf{x}} \mathbf{h}_{k}\right\|_{L^{\infty}} \leq\left\|\partial_{\mathbf{x}} \mathbf{h}\right\|_{L^{\infty}}+\|\mathbf{h}\|_{L^{\infty}}$ and (if $m=N) \sup _{k \in \mathbb{N}}\left\|\operatorname{div}_{\mathbf{x}} \mathbf{h}_{k}\right\|_{L^{\infty}} \leq\left\|\operatorname{div}_{\mathbf{x}} \mathbf{h}\right\|_{L^{\infty}}+\|\mathbf{h}\|_{L^{\infty}}$. 
In general, any proof via smoothing coefficients requires some limit process in the end, so we need the completeness of the underlying subset of $L^{p}\left(\mathbb{R}^{N}\right)$ with respect to $d_{L^{p}}$. As a consequence of Proposition 18, this feature is closely related to the tightness of the values to the power $p$ (of solutions to the approximate problems). The dominating function mentioned in Corollary 24 provides the tool for specifying which norms of the coefficients should be uniformly bounded in this context:

Lemma 31 (Tight values due to uniformly bounded velocities) Let $G \subset C_{c}^{1}\left(\mathbb{R}^{N}, \mathbb{R}^{N}\right.$ ) and $U \subset C^{1}\left(\mathbb{R}^{N}\right) \cap W^{1, \infty}\left(\mathbb{R}^{N}\right)$ be any nonempty subsets with $\gamma:=\sup \left\{\|\mathbf{g}\|_{L^{\infty}}+\right.$ $\left.\left\|\operatorname{div}_{\mathbf{x}} \mathbf{g}\right\|_{L^{\infty}}+\|u\|_{L^{\infty}} \mid \mathbf{g} \in G, \quad u \in U\right\}<\infty$. Suppose that $W:=\{w \in$ $L^{p}\left(\mathbb{R}^{N}\right)|| w \mid \leq \widehat{w}$ Lebesgue-a.e. in $\left.\mathbb{R}^{N} \backslash K_{\mathbf{x}}\right\}$ for some $\widehat{w} \in L^{p}\left(\mathbb{R}^{N}\right)$ and a compact set $K_{\mathbf{x}} \subset \mathbb{R}^{N}$.

Then for every $f_{0} \in L^{p}\left(\mathbb{R}^{N}\right)$, the subset $\left\{\left|\vartheta_{\mathbf{g}, u, w}^{f}\left(t, f_{0}\right)\right|^{p} \mid t \in[0,1], \mathbf{g} \in G\right.$, $u \in U, w \in W\} \subset L^{1}\left(\mathbb{R}^{N}\right)$ is tight, i.e., for $r \rightarrow \infty$,

$$
\sup \left\{\int_{\mathbb{R}^{N} \backslash \mathbb{B}_{r}(0)}\left|\vartheta_{\mathbf{g}, u, w}^{f}\left(t, f_{0}\right)\right|^{p} d \mathbf{x} \mid t \in[0,1], \mathbf{g} \in G, u \in U, w \in W\right\} \rightarrow 0 .
$$

Proof of Lemma 31 This is based on the simple observation that $\left|\mathfrak{X}_{1, \mathbf{g}}(t, \mathbf{x})-\mathbf{x}\right| \leq C t$ holds for all $\mathbf{x} \in \mathbb{R}^{N}$ and $t \geq 0$ with the constant $C:=\sup _{\mathbf{g} \in G}\|\mathbf{g}\|_{L^{\infty}}<\infty$.

Indeed, for every $\varepsilon>0$, there is a radius $\widehat{r}>0$ with $K_{\mathbf{x}} \subset \mathbb{B}_{\widehat{r}}(0)$ and $\left\|f_{0}\right\|_{L^{p}\left(\mathbb{R}^{N} \backslash \mathbb{B}_{\widehat{r}(0))}\right.}+\|\widehat{w}\|_{L^{p}\left(\mathbb{R}^{N} \backslash \mathbb{B}_{\vec{r}}(0)\right)}<\varepsilon$. Set $R:=\widehat{r}+C \geq \widehat{r}$ and $Z_{r}$ $:=\mathbb{R}^{N} \backslash \mathbb{B}_{r}(0) \subset \mathbb{R}^{N}$ for each $r \geq \widehat{r}$. Due to Corollary 24 , the function $\xi_{t}:=$ $\vartheta_{\mathbf{g}, u, w}^{f}\left(t, f_{0}\right) \in L^{p}\left(\mathbb{R}^{N}\right)$ with any $\mathbf{g} \in G, u \in U, w \in W, t \in[0,1]$ satisfies

$$
\begin{aligned}
\left\|\xi_{t}\right\|_{L^{p}\left(Z_{R}\right)}^{p}= & \int_{Z_{R}}\left|\xi_{t}(\mathbf{x})\right|^{p} d \mathbf{x} \\
\leq & c \cdot e^{c\left(\left\|\operatorname{div}_{\mathbf{x}} \mathbf{g}\right\|_{L^{\infty}}+\|u\|_{L^{\infty}}\right) \cdot t}\left(\int_{Z_{R}}\left|f_{0}\left(\mathfrak{X}_{-1, \mathbf{g}}(t, \mathbf{x})\right)\right|^{p} d \mathbf{x}\right. \\
& \left.+\int_{Z_{R}} \sup _{s \in[0, t]}\left|w\left(\mathfrak{X}_{-1, \mathbf{g}}(s, \mathbf{x})\right)\right|^{p} d \mathbf{x}\right) \\
\leq & c \cdot e^{c\left(2\left\|\operatorname{div}_{\mathbf{x}} \mathbf{g}\right\|_{\left.L^{\infty}+\|u\|_{L} \infty\right)} \cdot t\right.} \int_{Z_{\widehat{r}}}\left(\left|f_{0}\right|^{p}+|\widehat{w}|^{p}\right) d \mathbf{x}
\end{aligned}
$$

with constants $c=c(N, p) \geq 1$ because for each $k \in \mathbb{N}$ and $s \in[0,1]$, the diffeomorphism $\mathfrak{X}_{-1, \mathbf{g}}(s, \cdot): \mathbb{R}^{N} \rightarrow \mathbb{R}^{N}$ maps $Z_{R}$ in a subset of $Z_{\widehat{r}} \subset \mathbb{R}^{N}$. Hence we obtain $\left\|\xi_{t}\right\|_{L^{p}\left(Z_{R}\right)}^{p} \leq \operatorname{const}(\gamma, N, p) \cdot \varepsilon$ with a constant depending on the fixed bound $\gamma<\infty$, dimension $N$ and $p \in(1, \infty)$ (but not on $\mathbf{g}, u, w$ or $t \in[0,1]$ ).

Proof of Proposition 29 Choose sequences $\left(\mathbf{g}_{k}\right)_{k \in \mathbb{N}}$ in $C_{c}^{\infty}\left(\mathbb{R}^{N}, \mathbb{R}^{N}\right)$ and $\left(u_{k}\right)_{k \in \mathbb{N}}$ in $C_{c}^{\infty}\left(\mathbb{R}^{N}\right)$ converging to $\mathbf{g}, u$, respectively, as described in Lemma 30. According to Proposition 20, each related initial value problem

$$
\partial_{t} f_{k}+\operatorname{div}_{\mathbf{x}}\left(f_{k} \mathbf{g}_{k}\right)=u_{k} f_{k}+w \text { in }[0, T], \quad f_{k}(0)=f_{0}
$$


has a weak solution $f_{k}:[0, T] \rightarrow L^{p}\left(\mathbb{R}^{N}\right)$. Estimates (3), (5) and (8) in Proposition 27 imply

$$
\left\|f_{k}(t)\right\|_{L^{p}} \leq e^{C T} \cdot\left(\left\|f_{0}\right\|_{L^{p}}+\|w\|_{L^{p}}\right), \quad e_{L^{p}}\left(f_{k}(s), f_{k}(t)\right) \leq C \cdot|t-s|
$$

with a constant $C=\operatorname{const}\left(N, p,\left\|f_{0}\right\|_{L^{p}},\|g\|_{L^{\infty}},\left\|\operatorname{div}_{\mathbf{x}} \mathbf{g}\right\|_{L^{\infty}},\|u\|_{L^{\infty}},\|w\|_{L^{p}}\right)$ for all $k \in \mathbb{N}, s, t \in[0, T]$ and

$$
\sup _{t \in[0, T]} d_{L^{p}}\left(f_{k}(t), f_{l}(t)\right) \rightarrow 0 \text { for } k, l \rightarrow \infty
$$

Furthermore, the subset $\left\{\left|f_{k}(t)\right|^{p} \mid k \in \mathbb{N}, t \in[0, T]\right\} \subset L^{1}\left(\mathbb{R}^{N}\right)$ is tight due to Lemma 31. According to Proposition 18, there exists a bounded limit function $f:[0, T] \rightarrow L^{p}\left(\mathbb{R}^{N}\right)$ with $d_{L^{p}}\left(f_{l}(t), f(t)\right) \rightarrow 0(l \rightarrow \infty)$ for each $t \in[0, T]$. By the triangle inequality, this convergence proves to be even uniform w.r.t. $t \in[0, T]$ :

$$
\sup _{t \in[0, T]} d_{L^{p}}\left(f_{k}(t), f(t)\right) \rightarrow 0 \quad(k \rightarrow \infty) .
$$

In particular, $f$ is Lipschitz continuous with respect to $\breve{e}_{L^{p}}$ and so it is weakly continuous due to Lemma 13.

Finally, $f:[0, T] \rightarrow L^{p}\left(\mathbb{R}^{N}\right)$ proves to satisfy the characteristic condition (6) on weak solutions, i.e.,

$$
\begin{aligned}
\int_{\mathbb{R}^{N}} \varphi\left(f\left(t_{2}\right)-f\left(t_{1}\right)\right) d \mathbf{x}= & \int_{t_{1}}^{t_{2}} \int_{\mathbb{R}^{N}}\left(f(s, \cdot)\left(\mathbf{g} \cdot \nabla_{\mathbf{x}} \varphi+u \varphi\right)\right) d \mathbf{x} d s \\
& +\left(t_{2}-t_{1}\right) \int_{\mathbb{R}^{N}} \varphi w d \mathbf{x}
\end{aligned}
$$

for any $0 \leq t_{1}<t_{2} \leq T$ and $\varphi \in C_{c}^{\infty}\left(\mathbb{R}^{N}\right)$. Indeed, the general relation $\breve{e}_{L^{p}} \leq d_{L^{p}}$ and Lemma 13 imply $f_{k}(t) \rightarrow f(t)$ weakly in $L^{p}\left(\mathbb{R}^{N}\right)$ for each $t \in[0, T]$. For arbitrary $\varepsilon>0$, choose the index $J \in \mathbb{N}$ sufficiently large such that $\left\|\mathbf{g}_{l}-\mathbf{g}\right\|_{L^{q}}+\left\|u_{l}-u\right\|_{L^{q}} \leq \varepsilon$ holds for all $l \geq J$. Fixing any indices $k, l \geq J$, the uniform convergence of $\left(f_{k}\right)_{k \in \mathbb{N}}$ w.r.t. $d_{L^{p}}$ leads to

$$
\begin{aligned}
& \left|\int_{t_{1}}^{t_{2}} \int_{\mathbb{R}^{N}} f_{k}(s, \cdot) \mathbf{g}_{k} \cdot \nabla_{\mathbf{x}} \varphi d \mathbf{x}-\int_{t_{1}}^{t_{2}} \int_{\mathbb{R}^{N}} f(s, \cdot) \mathbf{g} \cdot \nabla_{\mathbf{x}} \varphi d \mathbf{x}\right| \\
& \leq\left|\int_{t_{1}}^{t_{2}} \int_{\mathbb{R}^{N}} f_{k}(s, \cdot)\left(\mathbf{g}_{k}-\mathbf{g}_{l}\right) \cdot \nabla_{\mathbf{x}} \varphi d \mathbf{x}\right| \\
& \quad+\left|\int_{t_{1}}^{t_{2}} \int_{\mathbb{R}^{N}}\left(f_{k}(s, \cdot)-f(s, \cdot)\right) \mathbf{g}_{l} \cdot \nabla_{\mathbf{x}} \varphi d \mathbf{x}\right| \\
& \quad+\left|\int_{t_{1}}^{t_{2}} \int_{\mathbb{R}^{N}} f(s, \cdot)\left(\mathbf{g}_{l}-\mathbf{g}\right) \cdot \nabla_{\mathbf{x}} \varphi d \mathbf{x}\right| \\
& \leq \operatorname{const}\left(N, p,\left\|f_{0}\right\|_{L^{p}},\|w\|_{L^{p}},\left\|\nabla_{\mathbf{x}} \varphi\right\|_{L^{\infty}}\right) \cdot\left\|\mathbf{g}_{k}-\mathbf{g}_{l}\right\|_{L^{q}}
\end{aligned}
$$




$$
\begin{aligned}
& +\operatorname{const}\left(N, p,\left\|\mathbf{g}_{l} \cdot \nabla_{\mathbf{x}} \varphi\right\|_{W^{1, \infty}}\right. \\
& \left.+\left\|\mathbf{g}_{l} \cdot \nabla_{\mathbf{x}} \varphi\right\|_{L^{q}}\right) \cdot \sup _{s \in[0, T]} d_{L^{p}}\left(f_{k}(s), f(s)\right) \\
& +\operatorname{const}\left(N, p,\left\|f_{0}\right\|_{L^{p}},\|w\|_{L^{p}},\left\|\nabla_{\mathbf{x}} \varphi\right\|_{L^{\infty}}\right) \cdot\left\|\mathbf{g}_{l}-\mathbf{g}\right\|_{L^{q}} .
\end{aligned}
$$

Hence we obtain

$$
\begin{aligned}
& \lim _{k \rightarrow \infty}\left|\int_{t_{1}}^{t_{2}} \int_{\mathbb{R}^{N}}\left(f_{k}(s, \cdot) \mathbf{g}_{k} \cdot \nabla_{\mathbf{x}} \varphi-f(s, \cdot) \mathbf{g} \cdot \nabla_{\mathbf{x}} \varphi\right) d \mathbf{x}\right| \\
& \quad \leq \operatorname{const}\left(\left\|f_{0}\right\|_{L^{p}},\|w\|_{L^{p}},\left\|\nabla_{\mathbf{x}} \varphi\right\|_{L^{\infty}}\right) \cdot \varepsilon .
\end{aligned}
$$

Essentially the same arguments guarantee

$$
\begin{aligned}
& \lim _{k \rightarrow \infty}\left|\int_{t_{1}}^{t_{2}} \int_{\mathbb{R}^{N}}\left(f_{k}(s, \cdot) u_{k} \varphi-f(s, \cdot) u \varphi\right) d \mathbf{x}\right| \\
& \quad \leq \operatorname{const}\left(\left\|f_{0}\right\|_{L^{p}},\|w\|_{L^{p}},\|\varphi\|_{L^{\infty}}\right) \cdot \varepsilon
\end{aligned}
$$

with $\varepsilon>0$ having been fixed arbitrarily small.

Finally the weak solution $f:[0, T] \rightarrow L^{p}\left(\mathbb{R}^{N}\right)$ is unique as a consequence of Proposition 19. The uniform convergence w.r.t $d_{L^{p}}$ implies that all estimates in Proposition 27 hold for $\vartheta_{\mathbf{g}, u, w}^{f}\left(\cdot, f_{0}\right) \stackrel{\text { Def. }}{=} f(\cdot):[0,1] \rightarrow L^{p}\left(\mathbb{R}^{N}\right)($ with $T=1)$.

\section{Euler compactness method for $L^{p}\left(\mathbb{R}^{N}\right)$-valued solutions}

Whenever we want to solve more complicated initial value problems by means of the linear autonomous problem (20), we have to rely on a method of approximation. There are usually two analytic bases between which we can choose: Completeness and compactness.

Proposition 18 gives sufficient conditions for the completeness of a subset of $L^{p}\left(\mathbb{R}^{N}\right)$ with respect to the metric $d_{L^{p}}$. In short, they consist of tightness and boundedness (w.r.t. the $L^{p}\left(\mathbb{R}^{N}\right)$ norm).

Here we are going to verify a form of sequential compactness which proves to be suitable for constructing solutions by means of Euler methods. In particular, this criterion is closely related to the observation that all Euler approximations are to start in the same initial state given. This motivated the term "Euler compact" in the general metric setting of mutational equations (see [51, Definition 2.15]).

Proposition 32 Fix $f_{0} \in L^{p}\left(\mathbb{R}^{N}\right), T>0, \gamma>0, \widehat{w} \in L^{p}\left(\mathbb{R}^{N}\right)$ and a compact set $K_{\mathbf{x}} \subset \mathbb{R}^{N}$ arbitrarily. Set

$$
\begin{gathered}
M_{\gamma, \widehat{w}}:=\left\{(\mathbf{g}, u, w) \mid \mathbf{g} \in L^{\infty}\left(\mathbb{R}^{N}, \mathbb{R}^{N}\right) \cap L^{q}, \partial_{\mathbf{x}} \mathbf{g} \in L^{\infty}, u \in L^{\infty}\left(\mathbb{R}^{N}\right) \cap L^{q},\right. \\
\nabla_{\mathbf{x}} u \in L^{\infty}\left(\mathbb{R}^{N}, \mathbb{R}^{N}\right), w \in L^{p}\left(\mathbb{R}^{N}\right),|w| \leq \widehat{w} \text { Leb.-a.e. in } \mathbb{R}^{N} \backslash K_{\mathbf{x}}, \\
\left.\|\mathbf{g}\|_{L^{\infty}}+\left\|\operatorname{div}_{\mathbf{x}} \mathbf{g}\right\|_{L^{\infty}}+\|u\|_{L^{\infty}}+\|w\|_{L^{p}} \leq \gamma\right\}
\end{gathered}
$$


and let $\mathcal{N}_{L^{p}}=\mathcal{N}_{L^{p}}\left(f_{0}, T, \gamma, \widehat{w}\right)$ denote the subset of all functions $f:[0, T] \rightarrow$ $L^{p}\left(\mathbb{R}^{N}\right)$ constructed in the following piecewise way: For an arbitrary equidistant partition $0=t_{0}<t_{1}<\cdots<t_{n}=T$ of $[0, T]$ and $n$ tuples $\left(\mathbf{g}_{k}, u_{k}, w_{k}\right) \in M_{\gamma}, \widehat{w}$ for $k=1, \ldots, n$, define $f:[0, T] \rightarrow L^{p}\left(\mathbb{R}^{N}\right)$ by means of the unique weak solution of the autonomous linear transport equation

$$
\partial_{t} f+\operatorname{div}_{\mathbf{x}}\left(f \mathbf{g}_{k}\right)=u_{k} f+w_{k} \text { in }\left[t_{k-1}, t_{k}\right], \quad f(0)=f_{0}
$$

(according to Proposition 29) in a continuous piecewise way, i.e., successively for $k=1, \ldots, n$.

Then the set $\left\{|f(t)|^{p} \mid t \in[0, T], f(\cdot) \in \mathcal{N}_{L^{p}}\right\} \subset L^{1}\left(\mathbb{R}^{N}\right)$ is tight. At each time $t \in[0, T]$, the set $\left\{f(t) \mid f(\cdot) \in \mathcal{N}_{L^{p}}\right\} \subset L^{p}\left(\mathbb{R}^{N}\right)$ is relatively compact w.r.t. the metric $d_{L^{p}}$.

The first statement of this proposition can be summarized briefly in the following way. The preceding Lemma 31 can be extended in two regards, namely from "more regular" to "less regular" coefficients and from just one autonomous linear problem to a piecewise autonomous linear problem. The first aspect is reflected by the choice of $M_{\gamma}, \widehat{w}$, while the second aspect refers to the construction of $\mathcal{N}_{L^{p}}\left(f_{0}, T, \gamma, \widehat{w}\right)$.

For the sake of transparency, we present an intermediate step first. Indeed, the proof of Lemma 31 is based essentially on Corollary 24 providing a function dominating the weak solution for "more regular" coefficients. For tightness, however, we need estimates for their integrals over complements of balls, as the proof of Lemma 31 reveals. This feature is concluded for solutions to the autonomous linear transport equation with "less regular" coefficients.

Lemma 33 (Integrals of powered weak solutions outside balls) Let the assumptions of Proposition 32 hold and consider any tuple $(\mathbf{g}, u, w) \in M_{\gamma}, \widehat{w}$. Then the weak solution $f:[0, T] \rightarrow L^{p}\left(\mathbb{R}^{N}\right)$ of the autonomous linear initial value problem (20) presented in Proposition 29 satisfies for every $t \in[0, T]$ and every radius $r>0$ with $K_{\mathbf{x}} \subset \mathbb{B}_{r}(0)$

$$
\int_{\mathbb{R}^{N} \backslash \mathbb{B}_{r+\gamma t}(0)}|f(t, \mathbf{x})|^{p} d \mathbf{x} \leq \operatorname{const}(N, p, T, \gamma) \cdot \int_{\mathbb{R}^{N} \backslash \mathbb{B}_{r}(0)}\left(\left|f_{0}\right|^{p}+|\widehat{w}|^{p}\right) d \mathbf{x}
$$

Proof of Lemma 33 The approximation method underlying the proof of Proposition 29 essentially bridges the gap between the autonomous linear transport equation with "more regular" coefficients and its counterpart with "less regular" coefficients. We choose the same approximating sequences $\left(\mathbf{g}_{k}\right)_{k \in \mathbb{N}}$ in $C_{c}^{\infty}\left(\mathbb{R}^{N}, \mathbb{R}^{N}\right)$ and $\left(u_{k}\right)_{k \in \mathbb{N}}$ in $C_{c}^{\infty}\left(\mathbb{R}^{N}\right)$ converging to $\mathbf{g}, u$ respectively as described in Lemma 30. Due to Proposition 20 , each related initial value problem

$$
\partial_{t} f_{k}+\operatorname{div}_{\mathbf{x}}\left(f_{k} \mathbf{g}_{k}\right)=u_{k} f_{k}+w \text { in }[0, T], \quad f_{k}(0)=f_{0}
$$


has a weak solution $f_{k}:[0, T] \rightarrow L^{p}\left(\mathbb{R}^{N}\right)$. Furthermore, we have already verified in the proof of Proposition 29

$$
\sup _{t \in[0, T]} d_{L^{p}}\left(f_{k}(t), f(t)\right) \rightarrow 0 \quad(k \rightarrow \infty) .
$$

For each $t \in[0, T]$, the general inequality $\breve{e}_{L^{p}} \leq d_{L^{p}}$ and Lemma 13 imply $f_{k}(t) \rightarrow$ $f(t)$ weakly in $L^{p}\left(\mathbb{R}^{N}\right)$, so the sequence of restrictions $\left(\left.f_{k}(t)\right|_{\mathbb{R}^{N} \backslash \mathbb{B}_{r+\gamma t}(0)}\right)_{k \in \mathbb{N}}$ converges weakly to $\left.f_{k}(t)\right|_{\mathbb{R}^{N} \backslash \mathbb{B}_{r+\gamma t}(0)}$ in $L^{p}\left(\mathbb{R}^{N} \backslash \mathbb{B}_{r+\gamma t}(0)\right)$. The norm is known to be lower semicontinuous w.r.t. weak convergence in a Banach space (see, e.g., [19, Proposition 3.5 (iii)]), thus

$$
\int_{\mathbb{R}^{N} \backslash \mathbb{B}_{r+\gamma t}(0)}|f(t, \mathbf{x})|^{p} d \mathbf{x} \leq \liminf _{k \rightarrow \infty} \int_{\mathbb{R}^{N} \backslash \mathbb{B}_{r+\gamma t}(0)}\left|f_{k}(t, \mathbf{x})\right|^{p} d \mathbf{x} .
$$

As in the proof of Lemma 31, we conclude from Corollary 24 for each $k \in \mathbb{N}$ and $t \in[0, T]$

$$
\begin{aligned}
& \int_{\mathbb{R}^{N} \backslash \mathbb{B}_{r+\gamma t}(0)}\left|f_{k}(t, \mathbf{x})\right|^{p} d \mathbf{x} \\
& \leq C \cdot\left(\int_{\mathbb{R}^{N} \backslash \mathbb{B}_{r+\gamma t}(0)}\left|f_{0}\left(\mathfrak{X}_{-1, \mathbf{g}_{k}}(t, \mathbf{x})\right)\right|^{p} d \mathbf{x}\right. \\
& \left.+\int_{\mathbb{R}^{N} \backslash \mathbb{B}_{r+\gamma t}(0)} \int_{0}^{t}\left|w\left(\mathfrak{X}_{-1, \mathbf{g}_{k}}(t-s, \mathbf{x})\right)\right|^{p} d s d \mathbf{x}\right)
\end{aligned}
$$

with a constant $C=C(N, p, T, \gamma)<\infty$ due to Hölder's inequality.

In the next step, we essentially use that $\left|\mathfrak{X}_{-1, \mathbf{g}_{k}}(t-s, \mathbf{x})-\mathbf{x}\right| \leq\left\|\mathbf{g}_{k}\right\|_{L^{\infty}}|t-s| \leq \gamma t$ holds for every $s \in[0, t]$ and $\mathbf{x} \in \mathbb{R}^{N}$. This implies $\mathfrak{X}_{-1, \mathbf{g}_{k}}(t-s, \mathbf{x}) \in \mathbb{R}^{N} \backslash \mathbb{B}_{r}(0)$ whenever $\mathbf{x} \in \mathbb{R}^{N} \backslash \mathbb{B}_{r+\gamma t}(0)$ and $s \in[0, T]$, so we conclude from the change of variables theorem for Lebesgue integrals that

$$
\begin{aligned}
& \int_{\mathbb{R}^{N} \backslash \mathbb{B}_{r+\gamma t}(0)}\left|f_{k}(t, \mathbf{x})\right|^{p} d \mathbf{x} \\
& \leq C \cdot\left(\int_{\mathbb{R}^{N} \backslash \mathbb{B}_{r+\gamma t}(0)}\left|f_{0}\left(\mathfrak{X}_{-1, \mathbf{g}_{k}}(t, \mathbf{x})\right)\right|^{p} d \mathbf{x}\right. \\
& \left.+\int_{0}^{t} \int_{\mathbb{R}^{N} \backslash \mathbb{B}_{r+\gamma t}(0)}\left|w\left(\mathfrak{X}_{-1, \mathbf{g}_{k}}(t-s, \mathbf{x})\right)\right|^{p} d \mathbf{x} d s\right) \\
& \leq C e^{\gamma t} \cdot\left(\int_{\mathbb{R}^{N} \backslash \mathbb{B}_{r}(0)}\left|f_{0}\right|^{p} d \mathbf{x}+\int_{0}^{t} \int_{\mathbb{R}^{N} \backslash \mathbb{B}_{r}(0)}|w|^{p} d \mathbf{x} d s\right) \\
& \leq C e^{\gamma t} \cdot\left(\int_{\mathbb{R}^{N} \backslash \mathbb{B}_{r}(0)}\left|f_{0}\right|^{p} d \mathbf{x}+t \cdot \int_{\mathbb{R}^{N} \backslash \mathbb{B}_{r}(0)}|\widehat{w}|^{p} d \mathbf{x}\right)
\end{aligned}
$$




$$
\leq C e^{(\gamma+1) t} \int_{\mathbb{R}^{N} \backslash \mathbb{B}_{r}(0)}\left(\left|f_{0}\right|^{p}+|\widehat{w}|^{p}\right) d \mathbf{x}
$$

Proof of Proposition 32 Consider any function $f:[0, T] \rightarrow L^{p}\left(\mathbb{R}^{N}\right)$ in $\mathcal{N}_{L^{p}}\left(f_{0}, T\right.$, $\gamma, \widehat{w})$. By definition, there are an underlying equidistant partition $0=t_{0}<t_{1}<\cdots<$ $t_{n}=T$ and $n$ tuples $\left(\mathbf{g}_{k}, u_{k}, w_{k}\right) \in M_{\gamma}, \widehat{w}$ for $k=1, \ldots, n$. We now apply the same arguments as in the preceding proof of Lemma 33 successively to the subintervals $\left[t_{k-1}, t_{k}\right]$ and, for every $t \in[0, T]$ and $r>0$ with $K_{\mathbf{x}} \subset \mathbb{B}_{r}(0)$, obtain

$$
\int_{\mathbb{R}^{N} \backslash \mathbb{B}_{r+\gamma t}(0)}|f(t, \mathbf{x})|^{p} d \mathbf{x} \leq \operatorname{const}(N, p, T, \gamma) \cdot \int_{\mathbb{R}^{N} \backslash \mathbb{B}_{r}(0)}\left(\left|f_{0}\right|^{p}+|\widehat{w}|^{p}\right) d \mathbf{x} .
$$

This slight extension of Lemma 33 to piecewise autonomous problems implies directly that the set $\left\{|f(t)|^{p} \mid t \in[0, T], f(\cdot) \in \mathcal{N}_{L^{p}}\right\} \subset L^{1}\left(\mathbb{R}^{N}\right)$ is tight.

Finally, at each time $t \in[0, T]$, the set $\left\{f(t) \mid f(\cdot) \in \mathcal{N}_{L^{p}}\right\} \subset L^{p}\left(\mathbb{R}^{N}\right)$ is norm bounded as a piecewise consequence of Proposition 27 (3) (extended to "less regular" coefficients). Hence its norm closed convex hull is relatively compact in $\left(L^{p}\left(\mathbb{R}^{N}\right), d_{L^{p}}\right)$ according to Corollary 16 and so is the set itself. This completes the proof of Euler compactness as specified in Proposition 32.

\subsection{Proofs of the well-posedness results in Sect. 3.1}

Now we consider the "full" nonlinear balance law (1) whose coefficients depend on the current $L^{p}$ integrable state in a functional (i.e., nonlocal) way as stated in Sect. 3.1:

$$
\partial_{t} f+\operatorname{div}_{\mathbf{x}}(\mathcal{G}(t, f) f)=\mathcal{U}(t, f) \cdot f+\mathcal{W}(t, f) \text { in }[0, T], \quad f(0)=f_{0}
$$

On the way to the existence of a weak solution, we first consider the stronger assumptions that all coefficient functions are continuous (w.r.t. both arguments) and not Carathéodory as assumed in Theorem 4. In particular, it is then more transparent to construct solutions by means of Euler method and the auxiliary solutions $\vartheta_{\mathbf{g}, u, w}^{f}:[0,1] \times L^{p}\left(\mathbb{R}^{N}\right) \rightarrow L^{p}\left(\mathbb{R}^{N}\right)$ to autonomous linear problems specified in Proposition 29.

Proposition 34 Consider the coefficient functions

$$
\begin{aligned}
& \mathcal{G}:[0, T] \times L^{p}\left(\mathbb{R}^{N}\right) \rightarrow\left\{\mathbf{g} \in L^{\infty}\left(\mathbb{R}^{N}, \mathbb{R}^{N}\right) \cap L^{q} \mid \partial_{\mathbf{y}} \mathbf{g} \in L^{\infty}\right\}, \\
& \mathcal{U}:[0, T] \times L^{p}\left(\mathbb{R}^{N}\right) \rightarrow\left\{u \in L^{\infty}\left(\mathbb{R}^{N}\right) \cap L^{q}\left(\mathbb{R}^{N}\right) \mid \nabla_{\mathbf{y}} u \in L^{\infty}\right\}, \\
& \mathcal{W}:[0, T] \times L^{p}\left(\mathbb{R}^{N}\right) \rightarrow L^{p}\left(\mathbb{R}^{N}\right),
\end{aligned}
$$

under the assumptions (i)-(iii) of Theorem 4 and the hypothesis

$\left(i v^{\prime}\right) \mathcal{G}, \mathcal{U}:[0, T] \times\left(L^{p}\left(\mathbb{R}^{N}\right), d_{L^{p}}\right) \rightarrow\left(L^{q},\|\cdot\|_{L^{q}}\right)$ and $\mathcal{W}:[0, T] \times$ $\left(L^{p}\left(\mathbb{R}^{N}\right), d_{L^{p}}\right) \rightarrow\left(L^{p},\|\cdot\|_{L^{p}}\right)$ are continuous. 
Then there exists a function $f:[0, T] \rightarrow L^{p}\left(\mathbb{R}^{N}\right)$ with the following properties:

(1) $f$ is bounded with respect to the $L^{p}\left(\mathbb{R}^{N}\right)$ norm,

(2) $f:[0, T] \rightarrow\left(L^{p}\left(\mathbb{R}^{N}\right), \breve{e}_{L^{p}}\right)$ is continuous,

(3) for a.e. $t \in\left[0, T\left[, \lim _{h \downarrow 0} \frac{1}{h} \cdot d_{L^{p}}\left(f(t+h), \vartheta_{(\mathcal{G}(t, f(t)), \mathcal{U}(t, f(t)), \mathcal{W}(t, f(t)))}^{f}(h, f(t))\right)\right.\right.$ $=0$,

(4) $f:[0, T] \rightarrow\left(L^{p}\left(\mathbb{R}^{N}\right)\right.$, weak $)$ is a continuous weak solution to initial value problem (1).

(5) $f$ is a renormalized solution (in the sense of DiPerna and Lions [33]) to the nonautonomous linear problem

$$
\partial_{t} f+\operatorname{div}_{\mathbf{x}}(f \widetilde{\mathbf{g}}(t))=\widetilde{u}(t) f+\widetilde{w}(t) \text { in }[0, T], \quad f(0)=f_{0}
$$

with the time-dependent coefficients $\widetilde{\mathbf{g}}:=\mathcal{G}(\cdot, f(\cdot)), \widetilde{u}:=\mathcal{U}(\cdot, f(\cdot)), \widetilde{w}:=$ $\mathcal{W}(\cdot, f(\cdot))$.

The proof of Proposition 34 consists of several steps:

Lemma 35 (A "mutational" solution) Under the assumptions of Proposition 34, there exists a function $f:[0, T] \rightarrow L^{p}\left(\mathbb{R}^{N}\right)$ with properties (1)-(3) specified there.

Proof It is essentially based on Euler method in combination with the suitable form of compactness. Let $\widetilde{C}>0$ denote the constant depending on $N, p, T, \gamma$ mentioned in Lemma 33. We follow the arguments in the proof of [51, Theorem 3.19] applied to the tuple $(E, d, e,\lfloor\cdot\rfloor)$ consisting of

$$
\begin{gathered}
E:=\left\{f \in L^{p}\left(\mathbb{R}^{N}\right) \mid \text { for every radius } \rho>0 \text { with } K_{\mathbf{x}} \subset \mathbb{B}_{\rho}(0) \subset \mathbb{R}^{N}:\right. \\
\left.\int_{\mathbb{R}^{N} \backslash \mathbb{B}_{\rho+\gamma T}(0)}|f|^{p} d \mathbf{x} \leq \widetilde{C} \cdot \int_{\mathbb{R}^{N} \backslash \mathbb{B}_{\rho}(0)}\left(\left|f_{0}\right|^{p}+|\widehat{w}|^{p}\right) d \mathbf{x}\right\}
\end{gathered}
$$

with $\lfloor\cdot\rfloor:=\|\cdot\|_{L^{p}\left(\mathbb{R}^{N}\right)}, d:=d_{L^{p}}$ (in the sense of Definition 1) and $e:=\breve{e}_{L^{p}}$ (in the sense of Definition 10).

On the basis of assumption (i) of Theorem 4, set the abbreviation

$$
\eta:=\sup _{t, f}\left(\left\|\operatorname{div}_{\mathbf{x}} \mathcal{G}(t, f)\right\|_{L^{\infty}}+\|\mathcal{U}(t, f)\|_{L^{\infty}}+\|\mathcal{W}(t, f)\|_{L^{p}}\right)<\infty
$$

Step 1. Construction of the Euler approximation $f_{n}:[0, T] \rightarrow E \subset L^{p}\left(\mathbb{R}^{N}\right)$.

For each index $n \in \mathbb{N}$ (with $2^{n}>T$ ), set $h_{n}:=\frac{T}{2^{n}}, t_{n}^{k}:=k h_{n}$ for $k=0, \ldots, 2^{n}$, $f_{n}(0):=f_{0} \in E$ and

$$
\begin{aligned}
f_{n}(t):= & \vartheta_{\left(\mathcal{G}\left(t_{n}^{k}, f\left(t_{n}^{k}\right)\right), \mathcal{U}\left(t_{n}^{k}, f\left(t_{n}^{k}\right)\right), \mathcal{W}\left(t_{n}^{k}, f\left(t_{n}^{k}\right)\right)\right)}^{f}\left(t-t_{n}^{k}, f\left(t_{n}^{k}\right)\right) \quad \text { for } t \in\left(t_{n}^{k}, t_{n}^{k+1}\right], \\
& 0 \leq k<2^{n} .
\end{aligned}
$$


This leads to a function $f_{n}:[0, T] \rightarrow L^{p}\left(\mathbb{R}^{N}\right)$. Proposition 27 (extended to "less regular" coefficients as mentioned in Proposition 29) implies in a piecewise way

- $\left\|f_{n}(t)\right\|_{L^{p}} \leq\left(\left\|f_{0}\right\|_{L^{p}}+\eta\right) \cdot e^{\operatorname{const}(N, \eta) \cdot T}=: \rho$ for all $t \in[0, T]$,

- $f_{n}:[0, T] \rightarrow\left(L^{p}\left(\mathbb{R}^{N}\right), \breve{e}\right)$ is $\lambda$-Lipschitz continuous with $\lambda=\lambda\left(N, \eta, \rho, C_{\rho}\right)$ where $C_{\rho}$ denotes the constant in hypothesis (ii) of Theorem 4.

Furthermore, we conclude from Lemma 33 in the same piecewise way as for Proposition 32

- $f_{n}(t) \in E$ holds for every $t \in[0, T]$ and $n \in \mathbb{N}$.

Step 2. Selecting a subsequence $\left(f_{n_{m}}\right)_{m \in \mathbb{N}}$ and its pointwise limit $f:[0, T] \rightarrow E$ w.r.t. $d_{L}{ }^{p}$.

Proposition 32 about "Euler compactness" states at each time $t \in[0, T]$ that the set $\left\{f_{n}(t) \mid n \in \mathbb{N}\right\}$ is relatively compact with respect to the metric $d_{L^{p}}$. Hence we can extract a subsequence $\left(f_{n_{m}}(t)\right)_{m \in \mathbb{N}}$ converging to some $f(t) \in L^{p}\left(\mathbb{R}^{N}\right)$ w.r.t. $d_{L^{p}}$. From $\breve{e}_{L^{p}} \leq d_{L^{p}}$ and Lemma 13 , this implies $f_{n_{m}}(t) \rightarrow f(t)$ weakly in $L^{p}\left(\mathbb{R}^{N}\right)$, so the lower semicontinuity of norms w.r.t. weak convergence leads to

$$
\begin{aligned}
\int_{\mathbb{R}^{N} \backslash \mathbb{B}_{\rho+\gamma T}(0)}|f(t, \mathbf{x})|^{p} d \mathbf{x} & \leq \liminf _{m \rightarrow \infty} \int_{\mathbb{R}^{N} \backslash \mathbb{B}_{\rho+\gamma T}(0)}\left|f_{n_{m}}(t, \mathbf{x})\right|^{p} d \mathbf{x} \\
& \leq \widetilde{C} \cdot \int_{\mathbb{R}^{N} \backslash \mathbb{B}_{\rho}(0)}\left(\left|f_{0}\right|^{p}+|\widehat{w}|^{p}\right) d \mathbf{x},
\end{aligned}
$$

i.e., hence $f(t) \in E$.

We now consider all rational $t \in[0, T]$ successively. Cantor's diagonal method provides both a subsequence (again denoted by) $\left(f_{n_{m}}\right)_{m \in \mathbb{N}}$ and a function $f$ : $[0, T] \cap \mathbb{Q} \rightarrow E \subset L^{p}\left(\mathbb{R}^{N}\right)$ such that $d_{L^{p}}\left(f_{n_{m}}(t), f(t)\right) \rightarrow 0(m \rightarrow \infty)$ for every $t \in[0, T] \cap \mathbb{Q}$. Then (even) for every $t \in[0, T] \backslash \mathbb{Q}$, the sequence $\left(f_{n_{m}}(t)\right)_{m \in \mathbb{N}}$ converges to some $f(t) \in E$ w.r.t. $d_{L^{p}}$. Indeed, fix $t \in[0, T] \backslash \mathbb{Q}$ arbitrarily. Due to Proposition 32, $\left(f_{n_{m}}(t)\right)_{m \in \mathbb{N}}$ has a point of accumulation $f(t) \in E$ w.r.t. $d_{L^{p}}$. It is now suffices to verify

$$
\lim _{k \rightarrow \infty} \breve{e}_{L^{p}}\left(f\left(s_{k}\right), f(t)\right)=0
$$

for any sequence $\left(s_{k}\right)_{k \in \mathbb{N}}$ in $[0, T] \cap \mathbb{Q}$ tending to $t$, because then the point of accumulation of $\left(f_{n_{m}}(t)\right)_{m \in \mathbb{N}}$ w.r.t. $d_{L^{p}}$ is unique and hence its limit. From the general relation $\breve{e}_{L^{p}} \leq d_{L^{p}}$, we conclude that

$$
\begin{aligned}
\breve{e}_{L^{p}}\left(f\left(s_{k}\right), f(t)\right) \leq & \breve{e}_{L^{p}}\left(f\left(s_{k}\right), f_{n_{m}}\left(s_{k}\right)\right)+\breve{e}_{L^{p}}\left(f_{n_{m}}\left(s_{k}\right), f_{n_{m}}(t)\right) \\
& +\breve{e}_{L^{p}}\left(f_{n_{m}}(t), f(t)\right) \\
\leq & d_{L^{p}}\left(f\left(s_{k}\right), f_{n_{m}}\left(s_{k}\right)\right)+\lambda \cdot\left|s_{k}-t\right|+d_{L^{p}}\left(f_{n_{m}}(t), f(t)\right) .
\end{aligned}
$$

In addition to $\lim _{m \rightarrow \infty} d_{L^{p}}\left(f\left(s_{k}\right), f_{n_{m}}\left(s_{k}\right)\right)=0$ for each $k \in \mathbb{N}$, the choice of $f(t) \in E$ implies that 


$$
\liminf _{m \rightarrow \infty} d_{L^{p}}\left(f_{n_{m}}(t), f(t)\right)=0
$$

Thus $\breve{e}_{L^{p}}\left(f\left(s_{k}\right), \quad f(t)\right) \leq \lambda \cdot\left|s_{k}-t\right|$ holds for every $k \in \mathbb{N}$ and converges to 0 for $k \rightarrow \infty$.

To summarise, we have now found a subsequence $\left(f_{n_{m}}\right)_{m \in \mathbb{N}}$ and a function $f$ : $[0, T] \rightarrow E \subset L^{p}\left(\mathbb{R}^{N}\right)$ such that

$$
d_{L^{p}}\left(f_{n_{m}}(t), f(t)\right) \rightarrow 0 \quad(m \rightarrow \infty)
$$

for every $t \in[0, T]$.

Step 3. Some estimates for this limit function $f:[0, T] \rightarrow E \subset L^{p}\left(\mathbb{R}^{N}\right)$. that

In view of Lemma 13 , the pointwise convergence of $\left(f_{n_{m}}\right)_{m \in \mathbb{N}}$ in $\left(E, d_{L^{p}}\right)$ implies

$$
f_{n_{m}}(t) \rightarrow f(t) \quad \text { weakly in } L^{p}\left(\mathbb{R}^{N}\right) \quad(m \rightarrow \infty)
$$

for every $t \in[0, T]$, so

$$
\|f(t)\|_{L^{p}} \leq \liminf _{m \rightarrow \infty}\left\|f_{n_{m}}(t)\right\|_{L^{p}} \leq \rho
$$

Furthermore we obtain for any $s, t \in[0, T]$ and $m \in \mathbb{N}$

$$
\begin{aligned}
\breve{e}_{L^{p}}(f(s), f(t)) \leq & \breve{e}_{L^{p}}\left(f(s), f_{n_{m}}(s)\right)+\breve{e}_{L^{p}}\left(f_{n_{m}}(s), f_{n_{m}}(t)\right) \\
& +\breve{e}_{L^{p}}\left(f_{n_{m}}(t), f(t)\right) \\
\leq & d_{L^{p}}\left(f(s), f_{n_{m}}(s)\right)+\lambda|s-t|+d_{L^{p}}\left(f_{n_{m}}(t), f(t)\right) .
\end{aligned}
$$

The limit for $m \rightarrow \infty$ yields $\breve{e}_{L^{p}}(f(s), \quad f(t)) \leq \lambda|s-t|$ for any $s, t \in[0, T]$, i.e., $f$ is $\lambda$-Lipschitz continuous w.r.t. $\breve{e}_{L^{p}}$. Hence, the claimed properties (1) and (2) (formulated in Proposition 34) are verified for $f$.

Step 4. $f$ also has the claimed property (3), i.e., for a.e. $t \in[0, T[$,

$$
\lim _{h \downarrow 0} \frac{1}{h} \cdot d_{L^{p}}\left(f(t+h), \vartheta_{(\mathcal{G}(t, f(t)), \mathcal{U}(t, f(t)), \mathcal{W}(t, f(t)))}^{f}(h, f(t))\right)=0 .
$$

Proposition 27 (8) specifies the dependence of solutions to autonomous linear problems (with "more regular" coefficients) on the coefficient functions. Similarly Proposition 27 (7) describes that the distance between initial states may increase at most exponentially.

Both estimates can be extended easily to "less regular" coefficients (as mentioned in Proposition 29) and to piecewise autonomous linear problems as they have just occurred in the construction of each Euler approximation $f_{n}:[0, T] \rightarrow E \subset$ $L^{p}\left(\mathbb{R}^{N}\right)$. 
Using the notation $[s]_{n}:=\max \left\{t_{n}^{k} \mid 0 \leq k \leq 2^{n}, t_{n}^{k} \leq s\right\}$ for any $s \in[0, T]$, we obtain for each $m \in \mathbb{N}$ and $t \in[0, T]$

$$
\begin{aligned}
& d_{L^{p}}\left(f_{n_{m}}(t+h), \vartheta_{(\mathcal{G}(t, f(t)), \mathcal{U}(t, f(t)), \mathcal{W}(t, f(t)))}^{f}(h, f(t))\right) \\
& \leq d_{L^{p}}\left(f_{n_{m}}(t+h), \vartheta_{(\mathcal{G}(t, f(t)), \mathcal{U}(t, f(t)), \mathcal{W}(t, f(t)))}^{f}\left(h, f_{n_{m}}(t)\right)\right) \\
& +d_{L^{p}}\left(\vartheta_{(\mathcal{G}(t, f(t)), \mathcal{U}(t, f(t)), \mathcal{W}(t, f(t)))}^{f}\left(h, f_{n_{m}}(t)\right),\right. \\
& \left.\quad \vartheta_{(\mathcal{G}(t, f(t)), \mathcal{U}(t, f(t)), \mathcal{W}(t, f(t)))}^{f}(h, f(t))\right) \\
& \leq \quad \operatorname{const}\left(N, \eta, \rho, C_{\rho}\right) \cdot \int_{t}^{t+h}\left(\left\|\mathcal{G}\left([s]_{n_{m}}, f_{n_{m}}\left([s]_{n_{m}}\right)\right)-\mathcal{G}(t, f(t))\right\|_{L^{q}}\right. \\
& +\left\|\mathcal{U}\left([s]_{n_{m}}, f_{n_{m}}\left([s]_{n_{m}}\right)\right)-\mathcal{U}(t, f(t))\right\|_{L^{q}} \\
& \left.+\left\|\mathcal{W}\left([s]_{n_{m}}, f_{n_{m}}\left([s]_{n_{m}}\right)\right)-\mathcal{W}(t, f(t))\right\|_{L^{p}}\right) d s \\
& +d_{L^{p}}\left(f_{n_{m}}(t), f(t)\right) \cdot e^{\operatorname{const}\left(N, \eta, C_{\rho}\right) \cdot h} .
\end{aligned}
$$

For each $s \in[0, T]$, we will show that $d_{L^{p}}\left(f_{n_{m}}\left([s]_{n_{m}}\right), f(s)\right) \rightarrow 0(m \rightarrow \infty)$. Indeed, we know for every $m \in \mathbb{N}$ that

$$
\breve{e}_{L^{p}}\left(f_{n_{m}}\left([s]_{n_{m}}\right), f_{n_{m}}(s)\right) \leq \lambda\left|[s]_{n_{m}}-s\right| \leq \lambda \frac{T}{2^{n_{m}}} \rightarrow 0 \quad(m \rightarrow \infty) .
$$

Due to the tightness of all functions in $E$, Corollary 15 leads to $d_{L^{p}}\left(f_{n_{m}}\left([s]_{n_{m}}\right), f_{n_{m}}(s)\right)$ $\rightarrow 0$ for $m \rightarrow \infty$. Hence, the triangle inequality of $d_{L^{p}}$ and the construction of $f(\cdot)$ (as a pointwise limit) imply

$$
\begin{aligned}
d_{L^{p}}\left(f_{n_{m}}\left([s]_{n_{m}}\right), f(s)\right) \leq & d_{L^{p}}\left(f_{n_{m}}\left([s]_{n_{m}}\right), f_{n_{m}}(s)\right) \\
& +d_{L^{p}}\left(f_{n_{m}}(s), f(s)\right) \rightarrow 0+0 \quad(m \rightarrow \infty) .
\end{aligned}
$$

From the continuity of $\mathcal{G}, \mathcal{U}, \mathcal{W}$ for every $h \in[0, T-t]$ we conclude that

$$
\begin{aligned}
& d_{L^{p}}\left(f(t+h), \vartheta_{(\mathcal{G}(t, f(t)), \mathcal{U}(t, f(t)), \mathcal{W}(t, f(t)))}^{f}(h, f(t))\right) \\
& =\lim _{m \rightarrow \infty} d_{L^{p}}\left(f_{n_{m}}(t+h), \vartheta_{(\mathcal{G}(t, f(t)), \mathcal{U}(t, f(t)), \mathcal{W}(t, f(t)))}^{f}(h, f(t))\right) \\
& \leq \operatorname{const}\left(N, \eta, \rho, C_{\rho}\right) \cdot \int_{t}^{t+h}\left(\|\mathcal{G}(s, f(s))-\mathcal{G}(t, f(t))\|_{L^{q}}\right. \\
& \quad+\|\mathcal{U}(s, f(s))-\mathcal{U}(t, f(t))\|_{L^{q}} \\
& \left.\quad+\|\mathcal{W}(s, f(s))-\mathcal{W}(t, f(t))\|_{L^{p}}\right) d s+0 .
\end{aligned}
$$


Finally, the fundamental theorem of calculus guarantees that

$$
\lim _{h \downarrow 0} \frac{1}{h} \cdot d_{L^{p}}\left(f(t+h), \vartheta_{(\mathcal{G}(t, f(t)), \mathcal{U}(t, f(t)), \mathcal{W}(t, f(t)))}^{f}(h, f(t))\right)=0 .
$$

Lemma 36 (A weak solution) Under the assumptions of Proposition 34, every function $f:[0, T] \rightarrow L^{p}\left(\mathbb{R}^{N}\right)$ with properties (1)-(3) satisfies the properties (4) and (5) claimed in Proposition 34.

Proof Choose $\varphi \in C_{c}^{1}\left(\mathbb{R}^{N}\right)$ arbitrarily and define $\psi:[0, T] \rightarrow \mathbb{R}$ by $\psi(t)=$ $\int_{\mathbb{R}^{N}} \varphi(\mathbf{x}) f(t, \mathbf{x}) d \mathbf{x}$. Due to the Lipschitz continuity of $f$ w.r.t. $\breve{e}_{L^{p}}$ (assumed in property (2)), $\psi$ is Lipschitz continuous. Furthermore at Lebesgue-almost every time instant $t \in[0, T[$, we conclude from property (3) that

$$
\begin{aligned}
& \lim _{h \downarrow 0} \frac{1}{h} \cdot\left(\int_{\mathbb{R}^{N}} \varphi f(t+h) d \mathbf{x}-\int_{\mathbb{R}^{N}} \varphi \vartheta_{(\mathcal{G}(t, f(t)), \mathcal{U}(t, f(t)), \mathcal{W}(t, f(t)))}^{f}(h, f(t)) d \mathbf{x}\right) \\
& \leq \lim _{h \downarrow 0} \frac{\|\varphi\|_{L} q+\|\varphi\|_{L} \infty+\left\|\nabla_{\mathbf{x}} \varphi\right\|_{L} \infty}{h} \\
& \cdot d_{L^{p}}\left(f(t+h), \vartheta_{(\mathcal{G}(t, f(t)), \mathcal{U}(t, f(t)), \mathcal{W}(t, f(t)))}^{f}(h, f(t))\right)=0 .
\end{aligned}
$$

Hence,

$$
\begin{aligned}
\lim _{h \downarrow 0} \frac{\psi(t+h)-\psi(t)}{h}= & \lim _{h \downarrow 0} \frac{1}{h} \cdot\left(\int_{\mathbb{R}^{N}} \varphi f(t+h) d \mathbf{x}-\int_{\mathbb{R}^{N}} \varphi f(t) d \mathbf{x}\right) \\
= & \lim _{h \downarrow 0} \frac{1}{h} \cdot\left(\int_{\mathbb{R}^{N}} \varphi \vartheta_{(\mathcal{G}(t, f(t)), \mathcal{U}(t, f(t)), \mathcal{W}(t, f(t)))}^{f}(h, f(t)) d \mathbf{x}\right. \\
& \left.-\int_{\mathbb{R}^{N}} \varphi f(t) d \mathbf{x}\right) \\
\stackrel{(6)}{=} & \lim _{h \downarrow 0} \frac{1}{h} \cdot\left(\int _ { 0 } ^ { h } \int _ { \mathbb { R } ^ { N } } \left(\vartheta^{f}(\mathcal{G}(t, f(t)), \mathcal{U}(t, f(t)), \mathcal{W}(t, f(t)))\right.\right. \\
& \left(\mathcal{G}(t, f(t)) \cdot \nabla_{\mathbf{x}} \varphi+\mathcal{U}(t, f(t)) \varphi\right) d \mathbf{x} d s \\
& \left.+h \int_{\mathbb{R}^{N}} \varphi \mathcal{W}(t, f(t)) d \mathbf{x}\right),
\end{aligned}
$$

i.e., $\lim _{h \downarrow 0} \frac{\psi(t+h)-\psi(t)}{h}=\int_{\mathbb{R}^{N}} f(t)\left(\mathcal{G}(t, f(t)) \cdot \nabla_{\mathbf{x}} \varphi+\mathcal{U}(t, f(t)) \varphi\right) d \mathbf{x}+$ $\int_{\mathbb{R}^{N}} \varphi \mathcal{W}(t, f(t)) d \mathbf{x}$.

Rademacher's theorem implies condition (2) on weak solutions to initial value problem

$$
\partial_{t} f+\operatorname{div}_{\mathbf{x}}(\mathcal{G}(t, f) f)=\mathcal{U}(t, f) \cdot f+\mathcal{W}(t, f) \text { in }[0, T], \quad f(0)=f_{0}
$$


Finally consider the composed coefficient functions as in property (5) in Proposition 34

$$
\begin{aligned}
\widetilde{\mathbf{g}} & :=\mathcal{G}(\cdot, f(\cdot)):[0, T] \rightarrow\left\{\mathbf{g} \in L^{\infty}\left(\mathbb{R}^{N}, \mathbb{R}^{N}\right) \cap L^{q} \mid \partial_{\mathbf{y}} \mathbf{g} \in L^{\infty}\right\}, \\
\widetilde{u} & :=\mathcal{U}(\cdot, f(\cdot)):[0, T] \rightarrow\left\{u \in L^{\infty}\left(\mathbb{R}^{N}\right) \cap L^{q}\left(\mathbb{R}^{N}\right) \mid \nabla_{\mathbf{y}} u \in L^{\infty}\right\}, \\
\widetilde{w} & :=\mathcal{W}(\cdot, f(\cdot)):[0, T] \rightarrow L^{p}\left(\mathbb{R}^{N}\right) .
\end{aligned}
$$

They satisfy the regularity assumptions of Proposition 19, so the weak solution to the nonautonomous linear problem

$$
\partial_{t} f+\operatorname{div}_{\mathbf{x}}(f \widetilde{\mathbf{g}}(t))=\widetilde{u}(t) f+\widetilde{w}(t) \text { in }[0, T], \quad f(0)=f_{0}
$$

is unique. Hence, it is always a renormalized solution (in the sense of DiPerna and Lions [33]).

Now we extend the existence result in Proposition 34 from continuous coefficients $\mathcal{G}, \mathcal{U}, \mathcal{W}$ to Carathéodory functions as assumed in Theorem 4. This gap is bridged approximatively by means of the following Scorza-Dragoni theorem for metric spaces.

Lemma 37 ([65, Theorem 1]) Let $S$ be a compact Hausdorff topological space, $\mu$ a Radon measure on $S$ and $X, Y$ metric spaces. Suppose $X$ to be separable.

Then every Carathéodory function $g: S \times X \rightarrow Y$ satisfies the Scorza-Dragoni property, i.e., for every $\varepsilon>0$, there exists a closed subset $S_{\varepsilon} \subset S$ with $\mu\left(S \backslash S_{\varepsilon}\right)<\varepsilon$ such that the restriction $\left.g\right|_{S_{\varepsilon} \times X}$ is continuous.

Proof of Theorem 4 Fix $\varepsilon>0$ arbitrarily. The Scorza-Dragoni theorem for metric spaces (i.e., Lemma 37) provides a closed subset $J_{\varepsilon} \subset[0, T]$ with $\mathcal{L}^{1}\left([0, T] \backslash J_{\varepsilon}\right)<\varepsilon$ such that the restrictions

$$
\begin{aligned}
& \left.\mathcal{G}\right|_{J_{\varepsilon} \times L^{p}\left(\mathbb{R}^{N}\right)}: J_{\varepsilon} \times L^{p}\left(\mathbb{R}^{N}\right) \rightarrow\left\{\mathbf{g} \in L^{\infty}\left(\mathbb{R}^{N}, \mathbb{R}^{N}\right) \cap L^{q} \mid \partial_{\mathbf{y}} \mathbf{g} \in L^{\infty}\right\}, \\
& \left.\mathcal{U}\right|_{J_{\varepsilon} \times L^{p}\left(\mathbb{R}^{N}\right)}: J_{\varepsilon} \times L^{p}\left(\mathbb{R}^{N}\right) \rightarrow\left\{u \in L^{\infty}\left(\mathbb{R}^{N}\right) \cap L^{q}\left(\mathbb{R}^{N}\right) \mid \nabla_{\mathbf{y}} u \in L^{\infty}\right\}, \\
& \left.\mathcal{W}\right|_{J_{\varepsilon} \times L^{p}\left(\mathbb{R}^{N}\right)}: J_{\varepsilon} \times L^{p}\left(\mathbb{R}^{N}\right) \rightarrow L^{p}\left(\mathbb{R}^{N}\right)
\end{aligned}
$$

are continuous.

The complement of $J_{\varepsilon}$ in $[0, T]$ is open, so consists of (at most) countably many open intervals. This allows us to extend these restrictions in a linear way to continuous functions

$$
\begin{aligned}
\mathcal{G}_{\varepsilon}:[0, T] \times L^{p}\left(\mathbb{R}^{N}\right) \rightarrow\left\{\mathbf{g} \in L^{\infty}\left(\mathbb{R}^{N}, \mathbb{R}^{N}\right) \cap L^{q} \mid \partial_{\mathbf{y}} \mathbf{g} \in L^{\infty}\right\}, \\
\mathcal{U}_{\varepsilon}:[0, T] \times L^{p}\left(\mathbb{R}^{N}\right) \rightarrow\left\{u \in L^{\infty}\left(\mathbb{R}^{N}\right) \cap L^{q}\left(\mathbb{R}^{N}\right) \mid \nabla_{\mathbf{y}} u \in L^{\infty}\right\}, \\
\mathcal{W}_{\varepsilon}:[0, T] \times L^{p}\left(\mathbb{R}^{N}\right) \rightarrow L^{p}\left(\mathbb{R}^{N}\right),
\end{aligned}
$$

whose values share the norm bounds and dominating function with the original coefficients $\mathcal{G}, \mathcal{U}, \mathcal{W}$ specified in hypotheses (i)-(iii) (of Theorem 4). 
The modified coefficient functions $\mathcal{G}_{\varepsilon}, \mathcal{U}_{\varepsilon}, \mathcal{W}_{\varepsilon}$ satisfy the assumptions of Proposition 34 and so, there exists a weak solution $f_{\varepsilon}:[0, T] \rightarrow L^{p}\left(\mathbb{R}^{N}\right)$ with properties (1)-(5) specified there.

For $\varepsilon>0$ we next consider any sequence tending to 0 . Then the same arguments as in the proof of Lemma 35 lead to a (sub-) sequence $\left(\varepsilon_{k}\right)_{k \in \mathbb{N}}$ and a function $f:[0, T] \rightarrow$ $L^{p}\left(\mathbb{R}^{N}\right)$ such that for every $t \in[0, T]$,

$$
d_{L^{p}}\left(f_{\varepsilon_{k}}(t), f(t)\right) \rightarrow 0 \quad(k \rightarrow \infty)
$$

Let $\eta, \rho, C_{\rho}$ abbreviate the a priori bounds introduced in the proof of Lemma 35. The construction of $f_{\varepsilon_{k}}(\cdot)$ (as limit of Euler approximations) and Proposition 27 (7.), (8.) imply for every $k \in \mathbb{N}, t \in[0, T), h \geq 0$ (with $t+h \leq T$ ) and any "less regular" coefficients $\mathbf{g}, u, w$ (as in Proposition 29)

$$
\begin{aligned}
d_{L^{p}}\left(f_{\varepsilon_{k}}(t+h), \quad \vartheta_{\mathbf{g}, u, w}^{f}(h, f(t))\right) \\
\leq e^{C \cdot h} \cdot\left(d_{L^{p}}\left(f_{\varepsilon_{k}}(t), f(t)\right)\right. \\
\quad+C \cdot \int_{t}^{t+h}\left(\left\|\mathcal{G}_{\varepsilon_{k}}\left(s, f_{\varepsilon_{k}}(s)\right)-\mathbf{g}\right\|_{L^{q}}+\left\|\mathcal{U}_{\varepsilon_{k}}\left(s, f_{\varepsilon_{k}}(s)\right)-u\right\|_{L^{q}}\right. \\
\left.\left.\quad+\left\|\mathcal{W}_{\varepsilon_{k}}\left(s, f_{\varepsilon_{k}}(s)\right)-w\right\|_{L^{p}}\right) d s\right)
\end{aligned}
$$

with a constant $C=\operatorname{const}\left(\eta, \rho, C_{\rho}\right)>0$. For every $k \in \mathbb{N}$ and $t \in[0, T)$, the triangle inequality for $d_{L^{p}}$ leads to

$$
\begin{aligned}
& d_{L^{p}}\left(f(t+h), \quad \vartheta^{f} f(\mathcal{G}(t, f(t)), \mathcal{U}(t, f(t)), \mathcal{W}(t, f(t)))(h, f(t))\right) \\
& \leq d_{L^{p}}\left(f(t+h), \quad f_{\varepsilon_{k}}(t+h)\right)+d_{L^{p}}\left(f_{\varepsilon_{k}}(t+h), \quad \vartheta_{(\mathcal{G}(t, f(t)), \mathcal{U}(t, f(t)), \mathcal{W}(t, f(t)))}^{f}(h, f(t))\right) \\
& \leq d_{L^{p}}\left(f(t+h), \quad f_{\varepsilon_{k}}(t+h)\right)+d_{L^{p}}\left(f_{\varepsilon_{k}}(t), f(t)\right) \cdot e^{C h} \\
& \quad+C e^{C h} \cdot \int_{t}^{t+h}\left(\left\|\mathcal{G}_{\varepsilon_{k}}\left(s, f_{\varepsilon_{k}}(s)\right)-\mathcal{G}(t, f(t))\right\|_{L^{q}}+\left\|\mathcal{U}_{\varepsilon_{k}}\left(s, f_{\varepsilon_{k}}(s)\right)-\mathcal{U}(t, f(t))\right\|_{L^{q}}\right. \\
& \left.\quad+\left\|\mathcal{W}_{\varepsilon_{k}}\left(s, f_{\varepsilon_{k}}(s)\right)-\mathcal{W}(t, f(t))\right\|_{L^{p}}\right) d s .
\end{aligned}
$$

The limit for $k \rightarrow \infty$ provides every $t \in[0, T)$ and $h>0$ with $t+h \leq T$

$$
\begin{aligned}
& d_{L^{p}}\left(f(t+h), \quad \vartheta_{(\mathcal{G}(t, f(t)), \mathcal{U}(t, f(t)), \mathcal{W}(t, f(t)))}^{f}(h, f(t))\right) \\
& \leq C e^{C h} \cdot \int_{t}^{t+h}\left(\|\mathcal{G}(s, f(s))-\mathcal{G}(t, f(t))\|_{L^{q}}+\|\mathcal{U}(s, f(s))-\mathcal{U}(t, f(t))\|_{L^{q}}\right. \\
& \left.\quad+\|\mathcal{W}(s, f(s))-\mathcal{W}(t, f(t))\|_{L^{p}}\right) d s .
\end{aligned}
$$


As a consequence of general results about Lebesgue points, it can be verified indirectly that the limit for $k \rightarrow \infty$ implies for Lebesgue-almost every $t \in[0, T)$,

$$
\limsup _{h \downarrow 0} \frac{1}{h} \cdot d_{L^{p}}\left(f(t+h), \vartheta_{(\mathcal{G}(t, f(t)), \mathcal{U}(t, f(t)), \mathcal{W}(t, f(t)))}^{f}(h, f(t))\right) \leq 0,
$$

i.e., the limit function $f:[0, T] \rightarrow L^{p}\left(\mathbb{R}^{N}\right)$ has the properties (1)-(3) specified in Proposition 34. According to Lemma 36, it is then a weak solution to nonlinear initial value problem

$$
\partial_{t} f+\operatorname{div}_{\mathbf{x}}(\mathcal{G}(t, f) f)=\mathcal{U}(t, f) \cdot f+\mathcal{W}(t, f) \text { in }[0, T], \quad f(0)=f_{0} .
$$

Until now our way to weak solutions is essentially based on the construction of functions $f:[0, T] \rightarrow L^{p}\left(\mathbb{R}^{N}\right)$ which are bounded w.r.t. $L^{p}\left(\mathbb{R}^{N}\right)$, continuous w.r.t. $\breve{e}_{L^{p}}$ and satisfy the infinitesimal condition on distances

$$
\lim _{h \downarrow 0} \frac{1}{h} \cdot d_{L^{p}}\left(f(t+h), \vartheta_{(\mathcal{G}(t, f(t)), \mathcal{U}(t, f(t)), \mathcal{W}(t, f(t)))}^{f}(h, f(t))\right)=0 .
$$

Then Lemma 36 guarantees that $f$ is a weak solution to the full nonlinear transport equation (with nonlocal dependences). For our further conclusions about the wellposedness, we formulate the inverse implication.

Lemma 38 Suppose that the assumptions of Theorem 4 hold and that $f:[0, T] \rightarrow$ $L^{p}\left(\mathbb{R}^{N}\right)$ satisfies the following conditions:

(1) $f$ is bounded with respect to the $L^{p}\left(\mathbb{R}^{N}\right)$ norm,

(2) $f:[0, T] \rightarrow\left(L^{p}\left(\mathbb{R}^{N}\right)\right.$, weak $)$ is continuous,

(3) the composite coefficient functions

$$
\begin{aligned}
& \widetilde{\mathbf{g}}:=\mathcal{G}(\cdot, f(\cdot)):[0, T] \rightarrow\left\{\mathbf{g} \in L^{\infty}\left(\mathbb{R}^{N}, \mathbb{R}^{N}\right) \cap L^{q} \mid \partial_{\mathbf{y}} \mathbf{g} \in L^{\infty}\right\}, \\
& \widetilde{u}:=\mathcal{U}(\cdot, f(\cdot)):[0, T] \rightarrow\left\{u \in L^{\infty}\left(\mathbb{R}^{N}\right) \cap L^{q}\left(\mathbb{R}^{N}\right) \mid \nabla_{\mathbf{y}} u \in L^{\infty}\right\}, \\
& \widetilde{w}:=\mathcal{W}(\cdot, f(\cdot)):[0, T] \rightarrow L^{p}\left(\mathbb{R}^{N}\right)
\end{aligned}
$$

satisfy the measurability conditions underlying $\widetilde{\mathbf{g}} \in L^{\infty}\left(0, T ; L^{\infty}\right), \partial_{\mathbf{x}} \widetilde{\mathbf{g}} \in$ $L^{\infty}\left(0, T ; L^{\infty}\right), \tilde{u} \in L^{\infty}\left(0, T ; L^{\infty}\right)$ and $\widetilde{w} \in L^{1}\left(0, T ; L^{p}\right)$,

(4) $f:[0, T] \rightarrow L^{p}\left(\mathbb{R}^{N}\right)$ is a weak solution to initial value problem (1) (in the sense of Eq. (2) for any $0 \leq t_{1}<t_{2} \leq T$ and $\left.\varphi \in C_{c}^{1}\left(\mathbb{R}^{N}\right)\right)$.

Then, the set $\left\{|f(t, \cdot)|^{p} \mid t \in[0, T]\right\} \subset L^{1}\left(\mathbb{R}^{N}\right)$ is tight and $f$ is continuous w.r.t. the metrics $\breve{e}_{L^{p}}, d_{L^{p}}$. Moreover, $f$ fulfils the infinitesimal condition at Lebesgue-almost every time instant $t \in[0, T)$

$$
\lim _{h \downarrow 0} \frac{1}{h} \cdot d_{L^{p}}\left(f(t+h), \vartheta_{(\mathcal{G}(t, f(t)), \mathcal{U}(t, f(t)), \mathcal{W}(t, f(t)))}^{f}(h, f(t))\right)=0 .
$$


Proof The proof is carried out in three steps.

Step 1. The set $\left\{|f(t, \cdot)|^{p} \mid t \in[0, T]\right\} \subset L^{1}\left(\mathbb{R}^{N}\right)$ is tight.

In Remark 25, inequality (16) specifies a function dominating the weak solution $f$ Lebesgue-almost everywhere in $[0, T] \times \mathbb{R}^{N}$. Hence, essentially the same arguments as in the proof of Lemma 33 imply that

$$
\int_{\mathbb{R}^{N} \backslash \mathbb{B}_{\rho+\gamma t}(0)}|f(t, \mathbf{x})|^{p} d \mathbf{x} \leq \text { const } \cdot e^{\text {const } \cdot(\gamma+1) t} \cdot \int_{\mathbb{R}^{N} \backslash \mathbb{B}_{\rho}(0)}\left(\left|f_{0}\right|^{p}+|\widehat{w}|^{p}\right) d \mathbf{x}
$$

for every $t \in[0, T]$ and each radius $\rho>0$ with $K_{\mathbf{X}} \subset \mathbb{B}_{\rho}(0)$. This in turn implies the claimed tightness of $\left\{|f(t, \cdot)|^{p} \mid t \in[0, T]\right\} \subset L^{1}\left(\mathbb{R}^{N}\right)$ as explained in the proof of Proposition 32.

Step 2. $f:[0, T] \rightarrow L^{p}\left(\mathbb{R}^{N}\right)$ is continuous w.r.t. both $\breve{e}_{L^{p}}$ and $d_{L^{p}}$.

Weak continuity and tight values imply the continuity w.r.t. $\breve{e}_{L^{p}}$ according to Lemma 13. Due to Corollary 15 , this is equivalent to the continuity w.r.t. $d_{L^{p}}$.

Step 3. The infinitesimal condition on $f(t+\cdot)$ w.r.t. $d_{L^{p}}$.

The coefficient functions $\mathcal{G}, \mathcal{U}:[0, T] \times\left(L^{p}\left(\mathbb{R}^{N}\right), d_{L^{p}}\right) \rightarrow\left(L^{q},\|\cdot\|_{L^{q}}\right)$ and $\mathcal{W}:$ $[0, T] \times\left(L^{p}\left(\mathbb{R}^{N}\right), d_{L^{p}}\right) \rightarrow\left(L^{p},\|\cdot\|_{L^{p}}\right)$ are supposed to be Carathéodory functions (according to hypothesis (iv), (v) of Theorem 4). Hence the composed functions $\widetilde{\mathbf{g}}$, $\widetilde{u}:[0, T] \rightarrow\left(L^{q},\|\cdot\|_{L^{q}}\right)$ and $\widetilde{w}:[0, T] \rightarrow\left(L^{p},\|\cdot\|_{L^{p}}\right)$ are measurable. Proposition 34 ensures the existence of a function $\bar{f}:[0, T] \rightarrow L^{p}\left(\mathbb{R}^{N}\right)$ satisfying

(1) $\bar{f}$ is bounded with respect to the $L^{p}\left(\mathbb{R}^{N}\right)$ norm,

(2) $\bar{f}:[0, T] \rightarrow\left(L^{p}\left(\mathbb{R}^{N}\right), \breve{e}_{L^{p}}\right)$ is Lipschitz continuous,

(3) $\lim _{h \downarrow 0} \frac{1}{h} \cdot d_{L^{p}}\left(\bar{f}(t+h), \vartheta_{(\widetilde{\mathbf{g}}(t), \widetilde{u}(t), \widetilde{w}(t))}^{f}(h, \bar{f}(t))\right)=0$ for a.e. $t \in[0, T)$,

(4) $\bar{f}:[0, T] \rightarrow\left(L^{p}\left(\mathbb{R}^{N}\right)\right.$, weak $)$ is a continuous weak solution to nonautonomous linear problem

$$
\partial_{t} \bar{f}+\operatorname{div}_{\mathbf{x}}(\bar{f} \widetilde{\mathbf{g}}(t))=\widetilde{u}(t) \bar{f}+\widetilde{w}(t) \text { in }[0, T], \quad \bar{f}(0)=f_{0} .
$$

According to Proposition 19, the weak solution to the last nonautonomous linear problem is unique, so $\bar{f}$ must coincide with $f$, i.e., $f(t)=\bar{f}(t) \in L^{p}\left(\mathbb{R}^{N}\right)$ for each $t \in[0, T]$. Property (3) gives the claimed infinitesimal condition on $f(t+\cdot)$ for Lebesgue-almost every $t \in[0, T)$.

Proof of Proposition 5 As a consequence of Lemma 38, each continuous weak solution $f:[0, T] \rightarrow\left(L^{p}\left(\mathbb{R}^{N}\right)\right.$, weak $)$ of the full nonlinear problem

$$
\partial_{t} f+\operatorname{div}_{\mathbf{x}}(\mathcal{G}(t, f) f)=\mathcal{U}(t, f) \cdot f+\mathcal{W}(t, f) \text { in }[0, T], \quad f(0)=f_{0}
$$

considered here in Proposition 5 also satisfies the properties (1)-(3) and (5) listed in Proposition 34. Furthermore, the set $\left\{|f(t)|^{p} \mid t \in[0, T]\right\} \subset L^{1}\left(\mathbb{R}^{N}\right)$ are tight, so $f$ is also continuous w.r.t. $d_{L^{p}}$ due to Corollary 15 . 
Let $f, \bar{f}:[0, T] \rightarrow L^{p}\left(\mathbb{R}^{N}\right)$ be two arbitrary solutions to the nonlinear problem sharing the initial state $f(0)=f_{0}=\bar{f}(0) \in L^{p}\left(\mathbb{R}^{N}\right)$. Then the function $\psi$ : $[0, T] \rightarrow[0, \infty)$ defined by $\psi(t)=d_{L^{p}}(f(t), \bar{f}(t))$ is continuous with $\psi(0)=0$ and satisfies, for Lebesgue-almost every $t \in[0, T)$,

$$
\begin{aligned}
& \underset{h \downarrow 0}{\limsup } \frac{\psi(t+h)-\psi(t)}{h}=\limsup _{h \downarrow 0} \frac{1}{h} \cdot\left(d_{L^{p}}(f(t+h), \bar{f}(t+h))-d_{L^{p}}(f(t), \bar{f}(t))\right) \\
& \leq \underset{h \downarrow 0}{\limsup } \frac{1}{h} \cdot d_{L^{p}}\left(f(t+h), \quad \vartheta_{(\mathcal{G}(t, f(t)), \mathcal{U}(t, f(t)), \mathcal{W}(t, f(t)))}^{f}(h, f(t))\right) \\
& \quad+\underset{h \downarrow 0}{\limsup } \frac{1}{h} \cdot\left(d _ { L ^ { p } } \left(\vartheta_{(\mathcal{G}(t, f(t)), \mathcal{U}(t, f(t)), \mathcal{W}(t, f(t)))}^{f}(h, f(t)),\right.\right. \\
& \left.\left.\quad \vartheta_{(\mathcal{G}(t, f(t)), \mathcal{U}(t, f(t)), \mathcal{W}(t, f(t)))}^{f}(h, \bar{f}(t))\right)-d_{L^{p}}(f(t), \bar{f}(t))\right) \\
& +\limsup _{h \downarrow 0} \frac{1}{h} \cdot d_{L^{p}}\left(\vartheta_{(\mathcal{G}(t, f(t)), \mathcal{U}(t, f(t)), \mathcal{W}(t, f(t)))}^{f}(h, \bar{f}(t)),\right. \\
& \left.\quad \vartheta_{(\mathcal{G}(t, \bar{f}(t)), \mathcal{U}(t, \bar{f}(t)), \mathcal{W}(t, \bar{f}(t)))}^{f}(h, \bar{f}(t))\right) \\
& +\underset{h \downarrow 0}{\limsup } \frac{1}{h} \cdot d_{L^{p}}\left(\vartheta_{(\mathcal{G}(t, \bar{f}(t)), \mathcal{U}(t, \bar{f}(t)), \mathcal{W}(t, \bar{f}(t)))}^{f}(h, \bar{f}(t)), \bar{f}(t+h)\right) \\
& \leq 0+\limsup _{h \downarrow 0} \frac{1}{h} \cdot d_{L^{p}}(f(t), \bar{f}(t)) \cdot\left(e^{C h}-1\right) \\
& +C \cdot\left(\|\mathcal{G}(t, f(t))-\mathcal{G}(t, \bar{f}(t))\|_{L^{q}}+\|\mathcal{U}(t, f(t))-\mathcal{U}(t, \bar{f}(t))\|_{L^{q}}\right. \\
& \left.\quad+\|\mathcal{W}(t, f(t))-\mathcal{W}(t, \bar{f}(t))\|_{L^{p}}\right)+0
\end{aligned}
$$

due to Proposition 27 (7) and (8) (extended to "less regular" coefficients as mentioned in Proposition 29). Here the constant $C$ depends on $\rho:=\sup _{t \in[0, T]}\left\{\|f(t)\|_{L^{p}},\|\bar{f}(t)\|_{L^{p}}\right\}$ $<\infty$ and the bounds given in hypotheses (i) and (ii) of Theorem 4 .

Assumption (vi') about $\Lambda_{\rho}$-Lipschitz continuity of the coefficients w.r.t. state $d_{L^{p}}$ leads to

$$
\begin{aligned}
\limsup _{h \downarrow 0} \frac{\psi(t+h)-\psi(t)}{h} & \leq C \cdot d_{L^{p}}(f(t), \bar{f}(t))+C \cdot 3 \Lambda_{\rho} \cdot d_{L^{p}}(f(t), \bar{f}(t)) \\
& \leq C\left(1+3 \Lambda_{\rho}\right) \cdot \psi(t)
\end{aligned}
$$

for Lebesgue-almost every $t \in[0, T[$. Finally, Gronwall's inequality (e.g., [51, Proposition A.2] or [52, Corollary A.1]) implies $\psi(t)=0$ for every $t \in[0, T]$, i.e., the weak solutions $f$ and $\bar{f}$ coincide.

Proof of Proposition 6 It follows essentially the same track as the preceding proof of Proposition 5 about uniqueness and, it just takes the different coefficient functions into consideration. The basic analytical tool is Gronwall's inequality again. The auxiliary function $\psi:[0, T] \rightarrow[0, \infty)$ defined by $\psi(t):=d_{L^{p}}\left(f^{(1)}(t), f^{(2)}(t)\right)$ is continuous 
with $\psi(0)=d_{L^{p}}\left(f_{0}^{(1)}, f_{0}^{(2)}\right)$ and satisfies, for Lebesgue-almost every $t \in[0, T)$,

$$
\begin{aligned}
& \limsup _{h \downarrow 0} \frac{\psi(t+h)-\psi(t)}{h} \\
& =\underset{h \downarrow 0}{\limsup _{h}} \frac{1}{h} \cdot\left(d_{L^{p}}\left(f^{(1)}(t+h), f^{(2)}(t+h)\right)-d_{L^{p}}\left(f^{(1)}(t), f^{(2)}(t)\right)\right) \leq \cdots \\
& \quad \leq 0+\limsup _{h \downarrow 0} \frac{1}{h} \cdot d_{L^{p}}\left(f^{(1)}(t), f^{(2)}(t)\right) \cdot\left(e^{C h}-1\right) \\
& \quad+C \cdot\left(\left\|\mathcal{G}^{(1)}\left(t, f^{(1)}(t)\right)-\mathcal{G}^{(2)}\left(t, f^{(2)}(t)\right)\right\|_{L^{q}}\right. \\
& \quad+\left\|\mathcal{U}^{(1)}\left(t, f^{(1)}(t)\right)-\mathcal{U}^{(2)}\left(t, f^{(2)}(t)\right)\right\|_{L^{q}} \\
& \left.\quad+\left\|\mathcal{W}^{(1)}\left(t, f^{(1)}(t)\right)-\mathcal{W}^{(2)}\left(t, f^{(2)}(t)\right)\right\|_{L^{p}}\right)+0
\end{aligned}
$$

$\lim \sup \frac{\psi(t+h)-\psi(t)}{h}$

$h \downarrow 0$

$\leq C \cdot d_{L^{p}}\left(f^{(1)}(t), f^{(2)}(t)\right)$

$+C \cdot\left(\left\|\mathcal{G}^{(1)}\left(t, f^{(1)}(t)\right)-\mathcal{G}^{(1)}\left(t, f^{(2)}(t)\right)\right\|_{L^{q}}\right.$

$+\left\|\mathcal{G}^{(1)}\left(t, f^{(2)}(t)\right)-\mathcal{G}^{(2)}\left(t, f^{(2)}(t)\right)\right\|_{L^{q}}$

$+\left\|\mathcal{U}^{(1)}\left(t, f^{(1)}(t)\right)-\mathcal{U}^{(1)}\left(t, f^{(2)}(t)\right)\right\|_{L^{q}}$

$+\left\|\mathcal{U}^{(1)}\left(t, f^{(2)}(t)\right)-\mathcal{U}^{(2)}\left(t, f^{(2)}(t)\right)\right\|_{L^{q}}$

$+\left\|\mathcal{W}^{(1)}\left(t, f^{(1)}(t)\right)-\mathcal{W}^{(1)}\left(t, f^{(2)}(t)\right)\right\|_{L^{p}}$

$\left.+\left\|\mathcal{W}^{(1)}\left(t, f^{(2)}(t)\right)-\mathcal{W}^{(2)}\left(t, f^{(2)}(t)\right)\right\|_{L^{p}}\right)$

$\leq C \cdot d_{L^{p}}\left(f^{(1)}(t), f^{(2)}(t)\right)$

$+C \cdot\left(\Lambda_{\rho} \cdot d_{L^{p}}\left(f^{(1)}(t), f^{(2)}(t)\right)+\sup _{\zeta \in L^{p}\left(\mathbb{R}^{N}\right):}\left\|\mathcal{G}^{(1)}(t, \zeta)-\mathcal{G}^{(2)}(t, \zeta)\right\|_{L^{q}}\right.$ $\|\zeta\|_{L} p \leq \rho$

$$
\begin{aligned}
& +\Lambda_{\rho} \cdot d_{L^{p}}\left(f^{(1)}(t), f^{(2)}(t)\right)+\sup _{\substack{\zeta \in L^{p}\left(\mathbb{R}^{N}\right): \\
\|\zeta\|_{L^{p}} \leq \rho}}\left\|\mathcal{U}^{(1)}(t, \zeta)-\mathcal{U}^{(2)}(t, \zeta)\right\|_{L^{q}} \\
& \left.+\Lambda_{\rho} \cdot d_{L^{p}}\left(f^{(1)}(t), f^{(2)}(t)\right)+\sup _{\substack{\zeta \in L^{p}\left(\mathbb{R}^{N}\right): \\
\|\zeta\|_{L^{p}} \leq \rho}}\left\|\mathcal{W}^{(1)}(t, \zeta)-\mathcal{W}^{(2)}(t, \zeta)\right\|_{L^{p}}\right) \\
& \leq C\left(1+3 \Lambda_{\rho}\right) \cdot \psi(t)+C \cdot \Delta(t)
\end{aligned}
$$

with the bounded auxiliary function $\Delta:[0, T] \rightarrow[0, \infty)$ defined by

$$
\Delta(t):=\sup _{\substack{\zeta \in L^{p}\left(\mathbb{R}^{N}\right): \\\|\zeta\|_{L^{p}} \leq \rho}}\left\|\mathcal{G}^{(1)}(t, \zeta)-\mathcal{G}^{(2)}(t, \zeta)\right\|_{L^{q}}
$$




$$
\begin{aligned}
& +\sup _{\substack{\zeta \in L^{p}\left(\mathbb{R}^{N}\right): \\
\|\zeta\|_{L^{p}} \leq \rho}}\left\|\mathcal{U}^{(1)}(t, \zeta)-\mathcal{U}^{(2)}(t, \zeta)\right\|_{L^{q}} \\
& +\sup _{\substack{\zeta \in L^{p}\left(\mathbb{R}^{N}\right): \\
\|\zeta\|_{L^{p}} \leq \rho}}\left\|\mathcal{W}^{(1)}(t, \zeta)-\mathcal{W}^{(2)}(t, \zeta)\right\|_{L^{p}},
\end{aligned}
$$

which is also Lebesgue measurable due to hypothesis (iv) of Theorem 4. Hence, by Gronwall's inequality,

$$
\psi(t) \leq \psi(0) \cdot e^{C\left(1+3 \Lambda_{\rho}\right) \cdot t}+\int_{0}^{t} \Delta(s) \cdot e^{C\left(1+3 \Lambda_{\rho}\right) \cdot(t-s)} d s
$$

for every $t \in[0, T]$.

\section{Measure-valued solutions to linear problems}

Here we investigate distributional solutions to nonlinear balance laws whose values are Radon measures on $\mathbb{R}^{N}$. From the analytical point of view, we follow the arguments in [51, Section 2.5] right away and give enough details for a self-contained presentation.

The basic idea is again to break the problem up into the family of autonomous linear problems and "feedback" via the Euler algorithm, exactly as for solutions with values in $L^{p}\left(\mathbb{R}^{N}\right)$ (in Sect. 4, 5). We simply adapt the classes of test functions when specifying the metric and now take the total variation into consideration. This special choice of test functions proved to be useful for structured population models in [38].

\subsection{Key features of the $W^{1, \infty}$ dual metric $d_{\mathcal{M}}$ on Radon measures $\mathcal{M}\left(\mathbb{R}^{N}\right)$}

The weak* topology on $\mathcal{M}\left(\mathbb{R}^{N}\right)$ is a rather obvious choice. There is, however, a very useful alternative which proves to be equivalent if we restrict our considerations to subsets of Radon measures which are "concentrated not too far away from each other".

Definition 39 A nonempty subset $\mathcal{S} \subset \mathcal{M}\left(\mathbb{R}^{N}\right.$ ) is called (uniformly) tight if for every $\varepsilon>0$ there exists a compact set $K_{\varepsilon} \subset \mathbb{R}^{N}$ such that the total variation of each $\mu \in \mathcal{S}$ satisfies $\sup _{\mu \in \mathcal{S}}|\mu|\left(\mathbb{R}^{N} \backslash K_{\varepsilon}\right)<\varepsilon$.

Tightness is just one of the many concepts which are often introduced (merely) for probability measures or positive Radon measures (see e.g. [4,5,8]). Many results also hold in $\mathcal{M}\left(\mathbb{R}^{N}\right)$ by considering the total variation (if necessary). Here we want to dispense with any global restrictions about the sign or total variation of Radon measures, so we cannot simply use, in particular, any Wasserstein metric (for probability measures).

The narrow topology on every tight subset of $\mathcal{M}\left(\mathbb{R}^{N}\right)$ is equivalent to the weak* topology (with respect to $\left.\mathcal{M}\left(\mathbb{R}^{N}\right)=C_{0}^{0}\left(\mathbb{R}^{N}\right)^{\prime}\right)$. It is metrizable on tight subsets with uniformly bounded total variation. 
Proposition 40 (1) For every $\lambda>0$ and $\mu, v \in \mathcal{M}\left(\mathbb{R}^{N}\right)$,

$$
\begin{aligned}
d_{\mathcal{M}}(\mu, v) & =\sup \left\{\frac{1}{\lambda} \int_{\mathbb{R}^{N}} \varphi d(\mu-v) \mid \varphi \in C_{c}^{\infty}\left(\mathbb{R}^{N}\right),\|\varphi\|_{L^{\infty}} \leq \lambda,\|\nabla \varphi\|_{L^{\infty}} \leq \lambda\right\} \\
& =\sup \left\{\frac{1}{\lambda} \int_{\mathbb{R}^{N}} \varphi d(\mu-v) \mid \varphi \in W^{1, \infty}\left(\mathbb{R}^{N}\right),\|\varphi\|_{L^{\infty}} \leq \lambda,\|\nabla \varphi\|_{L^{\infty}} \leq \lambda\right\} \\
& =\|\mu-v\|_{\left(W^{1, \infty}\right)^{\prime}}
\end{aligned}
$$

(2) For any tight sequence $\left(\mu_{n}\right)_{n \in \mathbb{N}}$ and $\mu$ in $\mathcal{M}\left(\mathbb{R}^{N}\right)$, the following equivalences hold

$$
\begin{aligned}
\lim _{n \rightarrow \infty} d_{\mathcal{M}}\left(\mu_{n}, \mu\right)=0 \& \sup _{n \in \mathbb{N}}\left|\mu_{n}\right|\left(\mathbb{R}^{N}\right)<\infty & \Leftrightarrow \mu_{n} \rightarrow \mu \text { weak } * \quad(n \rightarrow \infty) \\
& \Leftrightarrow \mu_{n} \rightarrow \mu \text { narrowly }(n \rightarrow \infty) .
\end{aligned}
$$

(3) The set $\left\{\mu \in \mathcal{M}\left(\mathbb{R}^{N}\right)|| \mu \mid\left(\mathbb{R}^{N}\right) \leq r\right\}$ is complete w.r.t. $d_{\mathcal{M}}$ for any $r>0$.

(4) Every tight set $\mathcal{S} \subset \mathcal{M}\left(\mathbb{R}^{N}\right)$ with $\sup _{\mu \in \mathcal{S}}|\mu|\left(\mathbb{R}^{N}\right)<\infty$ is relatively compact w.r.t. $d_{\mathcal{M}}$

Remark 41 The metric $d_{\mathcal{M}}$ on Radon measures (specified in Definition 3) is known under various names in the literature. It is called Kantorovich-Rubinshtein metric in [15, Section 8.3], $W^{1, \infty}$ dual metric in [51, Section 2.5.1]. Statement (1) in the recent proposition motivates us to prefer the second name. Equivalent counterparts can be found as bounded Lipschitz a.k.a. Fortet-Mourier distance (originally for probability measures) in [66, Section 6] or as flat metric of finite Radon measures on $\mathbb{R}^{+}$and $\mathbb{R}^{N}$ respectively in $[20,21,38]$, for example.

From our point of view, the motivation of $d_{\mathcal{M}}$ has two origins: First, the theorem of Hahn-Banach leads to a representation of the well established total variation of a Radon measure $\mu$ on $\mathbb{R}^{N}$

$$
|\mu|\left(\mathbb{R}^{N}\right)=\sup \left\{\int_{\mathbb{R}^{N}} \varphi(x) d \mu(x) \mid \varphi \in C_{c}^{0}\left(\mathbb{R}^{N}\right),\|\varphi\|_{L^{\infty}} \leq 1\right\}
$$

similarly to Remark 12 about the $L^{p}$ norm. Second, the popular Wasserstein metric can be characterized both in terms of an optimal transport problem and by means of dual function. The latter has the form

$$
W_{1}(\mu, \nu)=\sup \left\{\int_{\mathbb{R}^{N}} \varphi(x) d \mu(x)-\int_{\mathbb{R}^{N}} \varphi(x) d \nu(x) \mid \varphi \in C^{0}\left(\mathbb{R}^{N}\right), \text { Lip } \varphi \leq 1\right\}
$$

but the underlying equivalence is restricted to probability measures $\mu, v$ (even on Polish metric space) in general (see, e.g., [15, Section 8.10], [66, Section 6]). The restriction to 1-Lipschitz continuous test functions $\varphi$ which are bounded in addition enables us to preserve the metric properties while generalizing the distance in two directions: First we can consider signed Radon measures (instead of positive ones) and second, the two compared Radon measures need not have the same total variations 
(as probability measures do). $d_{\mathcal{M}}$ (almost) suggests itself in this context, and we have already benefited from this notion in [38,51], for example.

Similarly to Sect. 4.1 about $L^{p}$ density functions, Proposition 40 serves the purpose of providing connections with more established concepts of convergence for Radon measures on $\mathbb{R}^{N}$ —restricted to tight subsets, at least.

Proof of Proposition 40 We essentially follow the arguments for [51, Proposition 2.43] and give all relevant details here for the sake of a self-contained presentation. (1) Considering the restrictions to an arbitrarily fixed compact subset of $\mathbb{R}^{N}$, each function in $W^{1, \infty}\left(\mathbb{R}^{N}\right)$ can be approximated by elements of $C_{c}^{\infty}\left(\mathbb{R}^{N}\right) \subset$ $C^{1}\left(\mathbb{R}^{N}\right) \cap W^{1, \infty}\left(\mathbb{R}^{N}\right)$ with respect to supremum norm. This implies the equivalent characterizations of $d_{\mathcal{M}}(\mu, v)$ asserted here.

(2) The equivalence of narrow and weak* convergence results from the assumption of tightness according to the comment preceding the statement of this proposition.

Now let $\left(\mu_{n}\right)_{n \in \mathbb{N}}$ be any sequence in $\mathcal{M}\left(\mathbb{R}^{N}\right)$ and $\mu \in \mathcal{M}\left(\mathbb{R}^{N}\right)$ satisfying $\lim _{n \rightarrow \infty} d_{\mathcal{M}}\left(\mu_{n}, \mu\right)=0$ and $\sup _{n \in \mathbb{N}}\left|\mu_{n}\right|\left(\mathbb{R}^{N}\right)<\infty$. In particular, $\int_{\mathbb{R}^{N}} \varphi d \mu_{n} \rightarrow \int_{\mathbb{R}^{N}} \varphi d \mu$ for $n \rightarrow \infty$ and every $\varphi \in W^{1, \infty}\left(\mathbb{R}^{N}\right)$. This sequence also converges for $\varphi \in C_{0}^{0}\left(\mathbb{R}^{N}\right)$ since $W^{1, \infty}\left(\mathbb{R}^{N}\right) \cap C_{0}^{0}\left(\mathbb{R}^{N}\right)$ is dense in $\left(C_{0}^{0}\left(\mathbb{R}^{N}\right),\|\cdot\|_{L^{\infty}}\right)$ and the total variations of $\left(\mu_{n}\right)_{n \in \mathbb{N}}$ are bounded. Thus, the sequence $\left(\mu_{n}\right)_{n \in \mathbb{N}}$ also converges weakly* in $\mathcal{M}\left(\mathbb{R}^{N}\right)=C_{0}^{0}\left(\mathbb{R}^{N}\right)^{\prime}$.

Finally, assume the tight sequence $\left(\mu_{n}\right)_{n \in \mathbb{N}}$ in $\mathcal{M}\left(\mathbb{R}^{N}\right)$ to converge weakly* to $\mu \in \mathcal{M}\left(\mathbb{R}^{N}\right)$. Then $C:=\sup _{n \in \mathbb{N}}\left|\mu_{n}\right|\left(\mathbb{R}^{N}\right)<\infty$ due to the uniform boundedness theorem (e.g., [68, Section V.1 Theorem 10]) and,

$$
|\mu|\left(\mathbb{R}^{N}\right) \leq \liminf _{n \rightarrow \infty}\left|\mu_{n}\right|\left(\mathbb{R}^{N}\right) \leq C
$$

We still have to prove for $n \rightarrow \infty$ that

$$
\sup \left\{\int_{\mathbb{R}^{N}} \varphi d\left(\mu_{n}-\mu\right) \mid \varphi \in C_{c}^{\infty}\left(\mathbb{R}^{N}\right),\|\varphi\|_{L^{\infty}} \leq 1,\|\nabla \varphi\|_{L^{\infty}} \leq 1\right\} \rightarrow 0
$$

Choose $\varepsilon>0$ arbitrarily. Then there exists a sufficiently large radius $R>0$ with

$$
\sup _{n \in \mathbb{N}}\left|\mu_{n}\right|\left(\mathbb{R}^{N} \backslash \mathbb{B}_{R}(0)\right)+|\mu|\left(\mathbb{R}^{N} \backslash \mathbb{B}_{R}(0)\right) \leq \varepsilon
$$

since $\left\{\mu_{n} \mid n \in \mathbb{N}\right\}$ is tight. Due to the Arzelà-Ascoli Theorem (see, e.g., [37]), the set $\left\{\varphi \in C_{c}^{\infty}\left(\mathbb{B}_{R+1}(0)\right) \mid\|\varphi\|_{L^{\infty}} \leq 1,\|\nabla \varphi\|_{L^{\infty}} \leq 1\right\}$ is relatively compact in $\left(C^{0}\left(\overline{\mathbb{B}}_{R+1}(0)\right),\|\cdot\|_{\infty}\right)$. Hence, there always exist finitely many functions $\widetilde{\varphi}_{1}, \ldots$, $\widetilde{\varphi}_{k_{\varepsilon}} \in C_{c}^{\infty}\left(\mathbb{R}^{N}\right)$ with support in $\mathbb{B}_{R+1}(0)$ and $\left\|\widetilde{\varphi}_{i}\right\|_{\infty} \leq 1,\left\|\nabla \widetilde{\varphi}_{i}\right\|_{\infty} \leq 1$ such that

$$
\begin{aligned}
& \left\{\varphi \in C_{c}^{\infty}\left(\mathbb{B}_{R+1}(0)\right) \mid\|\varphi\|_{L^{\infty}} \leq 1,\|\nabla \varphi\|_{L^{\infty}} \leq 1\right\} \\
& \subset \bigcup_{i=1 \ldots k_{\varepsilon}}\left\{\varphi\left|\left\|\varphi-\left.\widetilde{\varphi}_{i}\right|_{\mathbb{B}_{R+1}(0)}\right\|_{L^{\infty}} \leq \varepsilon\right\} .\right.
\end{aligned}
$$


This implies

$$
\begin{aligned}
& \sup \left\{\int_{\mathbb{R}^{N}} \varphi d\left(\mu_{n}-\mu\right) \mid \varphi \in C_{c}^{\infty}\left(\mathbb{R}^{N}\right),\|\varphi\|_{L^{\infty}} \leq 1,\|\nabla \varphi\|_{L^{\infty}} \leq 1\right\} \\
& \leq \sup \left\{\int_{\mathbb{B}_{R}(0)} \varphi d\left(\mu_{n}-\mu\right) \mid \varphi \in C_{c}^{\infty}\left(\mathbb{R}^{N}\right),\|\varphi\|_{L^{\infty}} \leq 1,\|\nabla \varphi\|_{\left.L^{\infty} \leq 1\right\}+\varepsilon}\right. \\
& \leq \sup \left\{\int_{\mathbb{B}_{R}(0)} \widetilde{\varphi}_{i} d\left(\mu_{n}-\mu\right) \mid 1 \leq i \leq k_{\varepsilon}\right\}+2 C \varepsilon+\varepsilon \\
& \leq \varepsilon+2 C \varepsilon+\varepsilon
\end{aligned}
$$

for all $n \in \mathbb{N}$ sufficiently large (merely depending on $\varepsilon$ ) since $\mu_{n} \rightarrow \mu$ weakly*.

(3) Let $\left(\mu_{n}\right)_{n \in \mathbb{N}}$ be a Cauchy sequence w.r.t. $d_{\mathcal{M}}$ satisfying $\sup _{n \in \mathbb{N}}\left|\mu_{n}\right|\left(\mathbb{R}^{N}\right) \leq$ $r<\infty$.

The arguments proving the first part " $\Longrightarrow$ " of statement (2) imply that $\left(\mu_{n}\right)_{n \in \mathbb{N}}$ is Cauchy sequence with respect to the weak* topology of $\mathcal{M}\left(\mathbb{R}^{N}\right)$. There is the unique measure $\mu \in \mathcal{M}\left(\mathbb{R}^{N}\right)$ as weak ${ }^{*}$ limit of $\left(\mu_{n}\right)_{n \in \mathbb{N}}$ due to [7, Theorem 1.59]. In particular, $|\mu|\left(\mathbb{R}^{N}\right) \leq \liminf _{n \rightarrow \infty}\left|\mu_{n}\right|\left(\mathbb{R}^{N}\right) \leq r$.

It remains to verify that $d_{\mathcal{M}}\left(\mu_{n}, \mu\right) \rightarrow 0$ for $n \rightarrow \infty$. Indeed for arbitrary $\varepsilon>0$, there exists an index $n_{\varepsilon} \in \mathbb{N}$ such that for all $m, n \geq n_{\varepsilon}$,

$$
\begin{aligned}
d_{\mathcal{M}}\left(\mu_{m}, \mu_{n}\right) & \stackrel{\text { Def. }}{=} \sup \left\{\int_{\mathbb{R}^{N}} \varphi d\left(\mu_{m}-\mu_{n}\right) \mid \varphi \in C_{c}^{\infty}\left(\mathbb{R}^{N}\right),\|\varphi\|_{L^{\infty}},\|\nabla \varphi\|_{L^{\infty}} \leq 1\right\} \\
& \leq \varepsilon .
\end{aligned}
$$

Due to the weak* convergence of $\left(\mu_{n}\right)_{n \in \mathbb{N}}$ to $\mu$ in $\mathcal{M}\left(\mathbb{R}^{N}\right)=\left(C_{0}^{0}\left(\mathbb{R}^{N}\right),\|\cdot\|_{\infty}\right)^{\prime}$, the limit for $n \rightarrow \infty$ reveals for every $m \geq n_{\varepsilon}$

$$
\begin{aligned}
d_{\mathcal{M}}\left(\mu_{m}, \mu\right) & \stackrel{\text { Def. }}{=} \sup \left\{\int_{\mathbb{R}^{N}} \varphi d\left(\mu_{m}-\mu\right) \mid \varphi \in C_{c}^{\infty}\left(\mathbb{R}^{N}\right),\|\varphi\|_{L^{\infty}},\|\nabla \varphi\|_{L^{\infty}} \leq 1\right\} \\
& \leq \sup \left\{\lim _{n \rightarrow \infty} \int_{\mathbb{R}^{N}} \varphi d\left(\mu_{m}-\mu_{n}\right) \mid \varphi \in C_{c}^{\infty}\left(\mathbb{R}^{N}\right),\|\varphi\|_{L^{\infty}},\|\nabla \varphi\|_{L^{\infty}} \leq 1\right\} \\
& \leq \varepsilon .
\end{aligned}
$$

(4) By the assumption of tightness, the relative compactness of $\mathcal{S}$ with respect to the metric $d_{\mathcal{M}}$ results from its weak* compactness in $\mathcal{M}\left(\mathbb{R}^{N}\right)=C_{0}^{0}\left(\mathbb{R}^{N}\right)^{\prime}$ and, the latter is ensured by the Banach-Alaoglu Theorem.

(Alternatively, the Prokhorov Theorem states that bounded and tight subsets of positive Radon measures are sequentially relatively compact with respect to narrow convergence $[5,8]$. An extension to Borel measures on a complete separable metric space can be found at [15, Theorem 8.6.2].) 


\subsection{Linear transport equations induce transitions on $\mathcal{M}\left(\mathbb{R}^{N}\right)$}

Among the transport equations for Radon measures, the linear one is much simpler to solve, of course. Indeed, the method of characteristics even provides an explicit solution to the initial value problem:

Let $\mathbf{b}: \mathbb{R}^{N} \rightarrow \mathbb{R}^{N}$ and $c: \mathbb{R}^{N} \rightarrow \mathbb{R}$ be bounded and Lipschitz continuous. For any given $v_{0} \in \mathcal{M}\left(\mathbb{R}^{N}\right)$, the linear problem here focuses on a measure-valued distributional solution $\mu:[0, T] \rightarrow \mathcal{M}\left(\mathbb{R}^{N}\right)$ defined by $t \mapsto \mu_{t}$ of

$$
\partial_{t} \mu_{t}+\operatorname{div}_{\mathbf{x}}\left(\mathbf{b} \mu_{t}\right)=c \mu_{t} \text { in }[0, T], \quad \mu_{0}=v_{0}
$$

in the sense that

$$
\int_{\mathbb{R}^{N}} \varphi d \mu_{t}-\int_{\mathbb{R}^{N}} \varphi d \nu_{0}=\int_{0}^{t} \int_{\mathbb{R}^{N}}(\nabla \varphi(\mathbf{x}) \cdot \mathbf{b}(\mathbf{x})+\varphi(\mathbf{x}) c(\mathbf{x})) d \mu_{s}(\mathbf{x}) d s
$$

for every $t \in[0, T]$ and any test function $\varphi \in C_{c}^{\infty}\left(\mathbb{R}^{N}, \mathbb{R}\right)$.

We use the notation introduced in Lemma $21: \mathfrak{X}_{1, \mathbf{b}}:[0, T] \times \mathbb{R}^{N} \rightarrow \mathbb{R}^{N}$ is induced by the flow along $\mathbf{b}$, i.e., $\mathfrak{X}_{1, \mathbf{b}}\left(\cdot, \mathbf{x}_{0}\right):[0, T] \rightarrow \mathbb{R}^{N}$ is the continuously differentiable solution to the Cauchy problem

$$
\frac{d}{d t} \mathbf{x}(t)=\mathbf{b}(\mathbf{x}(t)) \text { in }[0, T], \quad \mathbf{x}(0)=\mathbf{x}_{0} .
$$

As a well-known result about ordinary differential equations, solutions to Cauchy problems are continuously differentiable with respect to initial data and right-hand side if the vector field (on the right-hand side) is continuously differentiable and, the following estimates result from the corresponding integral equations and Gronwall's inequality (see, e.g., [39, Chapter V], [40, Chapter 17], [67, Section 13]).

Lemma 42 For any vector fields $\mathbf{b}, \overline{\mathbf{b}} \in C^{1}\left(\mathbb{R}^{N}, \mathbb{R}^{N}\right) \cap W^{1, \infty}\left(\mathbb{R}^{N}, \mathbb{R}^{N}\right)$ the solution maps $\mathfrak{X}_{1, \mathbf{b}}, \mathfrak{X}_{1, \overline{\mathbf{b}}}:[0, T] \times \mathbb{R}^{N} \rightarrow \mathbb{R}^{N}$ are continuously differentiable with

$$
\operatorname{Lip} \mathfrak{X}_{1, \mathbf{b}}(t, \cdot) \leq e^{\operatorname{Lipb} \cdot t}, \quad\left\|\mathfrak{X}_{1, \mathbf{b}}(t, \cdot)-\mathfrak{X}_{1, \overline{\mathbf{b}}}(t, \cdot)\right\|_{L^{\infty}} \leq\|\mathbf{b}-\overline{\mathbf{b}}\|_{L^{\infty}} \cdot t e^{t \cdot \operatorname{Lip} \overline{\mathbf{b}}} .
$$

Proposition 43 For any $\mathbf{b} \in W^{1, \infty}\left(\mathbb{R}^{N}, \mathbb{R}^{N}\right), c \in W^{1, \infty}\left(\mathbb{R}^{N}, \mathbb{R}\right)$ and initial measure $\nu_{0} \in \mathcal{M}\left(\mathbb{R}^{N}\right)$, a solution $\mu:[0, T] \rightarrow \mathcal{M}\left(\mathbb{R}^{N}\right)$ written as $t \mapsto \mu_{t}$ to the linear problem

$$
\partial_{t} \mu_{t}+\operatorname{div}_{\mathbf{x}}\left(\mathbf{b} \mu_{t}\right)=c \mu_{t} \quad \text { in }[0, T], \quad \mu_{0}=v_{0}
$$

(in the distributional sense) is given by

$$
\int_{\mathbb{R}^{N}} \varphi d \mu_{t}=\int_{\mathbb{R}^{N}} \varphi\left(\mathfrak{X}_{1, \mathbf{b}}(t, \mathbf{x})\right) \cdot \exp \left(\int_{0}^{t} c\left(\mathfrak{X}_{1, \mathbf{b}}(s, \mathbf{x})\right) d s\right) d \nu_{0}(\mathbf{x})
$$

for all $\varphi \in C_{c}^{1}\left(\mathbb{R}^{N}\right)$. 
Proof First, we verify that the right-hand side provides a distributional solution to the linear problem with the initial measure $v_{0}$. In fact, it is absolutely continuous with respect to $t$ because for any subinterval $[s, t] \subset[0, T]$,

$$
\begin{aligned}
& \left|\int_{\mathbb{R}^{N}} \varphi d \mu_{t}-\int_{\mathbb{R}^{N}} \varphi d \mu_{s}\right| \\
& \quad=\left|\int_{\mathbb{R}^{N}}\left(\varphi\left(\mathfrak{X}_{1, \mathbf{b}}(t, \mathbf{x})\right) \cdot e^{\int_{0}^{t} c\left(\mathfrak{X}_{1, \mathbf{b}}(r, \mathbf{x})\right) d r}-\varphi\left(\mathfrak{X}_{1, \mathbf{b}}(s, \mathbf{x})\right) \cdot e^{\int_{0}^{s} c\left(\mathfrak{X}_{1, \mathbf{b}}(r, \mathbf{x})\right) d r}\right) d \mu_{0}(\mathbf{x})\right| \\
& \quad \leq \int_{\mathbb{R}^{N}}\left(\left|\left[\varphi\left(\mathfrak{X}_{1, \mathbf{b}}(\sigma, \mathbf{x})\right)\right]_{\sigma=s}^{\sigma=t}\right| e^{t\|c\|_{L^{\infty}}}+\left|\varphi\left(\mathfrak{X}_{1, \mathbf{b}}(s, \mathbf{x})\right)\right|\left[e^{\int_{0}^{\sigma} c\left(\mathfrak{X}_{1, \mathbf{b}}(r, \mathbf{x})\right) d r}\right]_{\sigma=s}^{\sigma=t}\right) d\left|\mu_{0}(\mathbf{x})\right| \\
& \quad \leq\left(\|\nabla \varphi\|_{L^{\infty}}\|\mathbf{b}\|_{L^{\infty}}(t-s) e^{t\|c\|_{L^{\infty}}}+\|\varphi\|_{L^{\infty}} e^{\left.t\|c\|_{L^{\infty}}\|c\|_{L^{\infty}}(t-s)\right)\left|\mu_{0}\right|\left(\mathbb{R}^{N}\right) .}\right.
\end{aligned}
$$

From the chain rule for weak derivatives, at Lebesgue-almost every time $t \in[0, T]$, we conclude that

$$
\begin{aligned}
& \frac{d}{d t} \int_{\mathbb{R}^{N}}\left(\varphi\left(\mathfrak{X}_{1, \mathbf{b}}(t, \mathbf{x})\right) \cdot \exp \left(\int_{0}^{t} c\left(\mathfrak{X}_{1, \mathbf{b}}(s, \mathbf{x})\right) d s\right)\right) d \nu_{0}(\mathbf{x}) \\
& =\int_{\mathbb{R}^{N}}\left(\nabla \varphi\left(\mathfrak{X}_{1, \mathbf{b}}(t, \mathbf{x})\right) \cdot \mathbf{b}\left(\mathfrak{X}_{1, \mathbf{b}}(t, \mathbf{x})\right)+\varphi\left(\mathfrak{X}_{1, \mathbf{b}}(t, \mathbf{x})\right) c\left(\mathfrak{X}_{1, \mathbf{b}}(t, \mathbf{x})\right)\right) \\
& \cdot e^{\int_{0}^{t} c\left(\mathfrak{X}_{1, \mathbf{b}}(r, \mathbf{x})\right) d r} d \nu_{0} \\
& =\int_{\mathbb{R}^{N}}(\nabla \varphi(\mathbf{y}) \cdot \mathbf{b}(\mathbf{y})+\varphi(\mathbf{y}) c(\mathbf{y})) d \mu_{t}(\mathbf{y}) .
\end{aligned}
$$

This solution is already well-known and usually denoted in the form of a push-forward. Furthermore, it is unique because solutions to the nonautonomous linear transport equation fulfil the following comparison principle (see also $[5,8,33]$ ):

Proposition 44 ([55] Lemma 3.5, Proposition 3.6) Let $v \in L^{1}\left([0, T] ; W^{1, \infty}\left(\mathbb{R}^{N}\right.\right.$, $\left.\mathbb{R}^{N}\right)$ ) be a Borel vector field and let $c(\cdot, \cdot)$ be a Borel bounded and locally Lipschitz continuous (w.r.t. the space variable) scalar function in $(0, T) \times \mathbb{R}^{N}$.

(1) For each probabilitymeasure $\widehat{\mu}_{0}$ on $\mathbb{R}^{N}$ (i.e., positive measure $\widehat{\mu}_{0} \in \mathcal{M}\left(\mathbb{R}^{N}\right.$ ) with $\left.\widehat{\mu}_{0}\left(\mathbb{R}^{N}\right)=1\right)$, there exists a unique narrowly continuous $\mu:[0, T] \rightarrow \mathcal{M}\left(\mathbb{R}^{N}\right)$, written $t \mapsto \mu_{t}$, solving the initial value problem (in the distributional sense)

$$
\partial_{t} \mu_{t}+\operatorname{div}_{\mathbf{x}}\left(v_{t} \mu_{t}\right)=c_{t} \mu_{t} \quad \text { in }(0, T) \times \mathbb{R}^{N}, \quad \mu_{0}=\widehat{\mu}_{0} .
$$

(2) The comparison principle holds in the following sense: Let $\sigma$, written $t \mapsto \sigma_{t}$, be a narrowly continuous family of (possibly signed) measures solving

$$
\partial_{t} \sigma_{t}+\operatorname{div}_{\mathbf{x}}\left(v_{t} \sigma_{t}\right)=c_{t} \sigma_{t} \quad \text { in }(0, T) \times \mathbb{R}^{N}
$$


with $\sigma_{0} \leq 0$ and

$$
\begin{aligned}
& \int_{0}^{T} \int_{\mathbb{R}^{N}}\left(\left|v_{t}(\mathbf{x})\right|+\left|c_{t}(\mathbf{x})\right|\right) d\left|\sigma_{t}\right|(\mathbf{x}) d t<\infty, \\
& \int_{0}^{T}\left(\left|\sigma_{t}\right|(B)+\sup _{B}\left|v_{t}\right|+\left.\operatorname{Lip} v_{t}\right|_{B}\right) d t<\infty \\
& \int_{0}^{T}\left(\left|\sigma_{t}\right|(B)+\sup _{B}\left|c_{t}\right|+\left.\operatorname{Lip} c_{t}\right|_{B}\right) d t<\infty
\end{aligned}
$$

for any bounded closed set $B \subset \mathbb{R}^{N}$. Then, $\sigma_{t} \leq 0$ holds for any $t \in[0, T)$.

The solutions to the linear problem lay the basis for transitions on $\mathcal{M}\left(\mathbb{R}^{N}\right)$ :

Definition 45 For each $\mathbf{b} \in W^{1, \infty}\left(\mathbb{R}^{N}, \mathbb{R}^{N}\right)$ and $c \in W^{1, \infty}\left(\mathbb{R}^{N}, \mathbb{R}\right)$, define $\vartheta_{\mathbf{b}, c}^{\mu}$ : $[0,1] \times \mathcal{M}\left(\mathbb{R}^{N}\right) \rightarrow \mathcal{M}\left(\mathbb{R}^{N}\right)$ by $\vartheta_{\mathbf{b}, c}^{\mu}\left(t, \mu_{0}\right)=\mu_{t}$ with $\mu:[0, T] \rightarrow \mathcal{M}\left(\mathbb{R}^{N}\right)$, written $t \mapsto \mu_{t}$, denoting the unique solution of

$$
\partial_{t} \mu_{t}+\operatorname{div}_{\mathbf{x}}\left(\mathbf{b} \mu_{t}\right)=c \mu_{t} \text { in }[0, T]
$$

(in the distributional sense) as specified in Proposition 43.

Proposition 46 For any $\mathbf{b}, \overline{\mathbf{b}} \in C^{1}\left(\mathbb{R}^{N}, \mathbb{R}^{N}\right) \cap W^{1, \infty}\left(\mathbb{R}^{N}, \mathbb{R}^{N}\right)$ and $c, \bar{c} \in$ $W^{1, \infty}\left(\mathbb{R}^{N}, \mathbb{R}\right)$ the measure-valued maps

$$
\vartheta_{\mathbf{b}, c}^{\mu}, \vartheta_{\overline{\mathbf{b}}, \bar{c}}^{\mu}:[0,1] \times \mathcal{M}\left(\mathbb{R}^{N}\right) \rightarrow \mathcal{M}\left(\mathbb{R}^{N}\right)
$$

fulfil for any $\mu_{0}, v_{0} \in \mathcal{M}\left(\mathbb{R}^{N}\right)$ and $t, h \in[0,1]$ with $t+h \leq 1$

(1) $\vartheta_{\mathbf{b}, c}^{\mu}\left(0, \mu_{0}\right)=\mu_{0}$

(2) $\vartheta_{\mathbf{b}, c}^{\mu}\left(h, \vartheta_{\mathbf{b}, c}^{\mu}\left(t, \mu_{0}\right)\right)=\vartheta_{\mathbf{b}, c}^{\mu}\left(t+h, \mu_{0}\right)$

(3) $\left|\vartheta_{\mathbf{b}, c}^{\mu}\left(h, \mu_{0}\right)\right|\left(\mathbb{R}^{N}\right) \leq e^{\|c\|_{L^{\infty} h}} \cdot\left|\mu_{0}\right|\left(\mathbb{R}^{N}\right)$

(4) $d_{\mathcal{M}}\left(\vartheta_{\mathbf{b}, c}^{\mu}\left(t, \mu_{0}\right), \vartheta_{\mathbf{b}, c}^{\mu}\left(t+h, \mu_{0}\right)\right) \leq h\left(\|\mathbf{b}\|_{L^{\infty}}+\|c\|_{L^{\infty}}\right) e^{\|c\|_{L^{\infty}}} \cdot\left|\mu_{0}\right|\left(\mathbb{R}^{N}\right)$

(5) $d_{\mathcal{M}}\left(\vartheta_{\mathbf{b}, c}^{\mu}\left(h, \mu_{0}\right), \vartheta_{\mathbf{b}, c}^{\mu}\left(h, v_{0}\right)\right) \leq d_{\mathcal{M}}\left(\mu_{0} v_{0}\right) \cdot e^{\left(\operatorname{Lip} \mathbf{b}+\|c\|_{W^{1, \infty}}\right) h}$

(6) $d_{\mathcal{M}}\left(\vartheta_{\mathbf{b}, c}^{\mu}\left(h, \quad \mu_{0}\right), \vartheta_{\overline{\mathbf{b}}, \bar{c}}^{\mu}\left(h, \mu_{0}\right)\right) \leq\left(\|\mathbf{b}-\overline{\mathbf{b}}\|_{L^{\infty}} \cdot e^{h\|\nabla c\|_{L^{\infty}}}+\|c-\bar{c}\|_{L^{\infty}}\right) h$ $e^{h \cdot\left(\operatorname{Lipb}+\max \left\{\|c\|_{L} \infty,\|\bar{c}\|_{L^{\infty}}\right\}\right)} \cdot\left|\mu_{0}\right|\left(\mathbb{R}^{N}\right)$.

Assuming $\mathbf{b}, \overline{\mathbf{b}} \in C^{1}\left(\mathbb{R}^{N}, \mathbb{R}^{N}\right)$ in addition to $\mathbf{b}, \overline{\mathbf{b}} \in W^{1, \infty}\left(\mathbb{R}^{N}, \mathbb{R}^{N}\right)$ serves the single purpose that we can use the estimates of the preceding Lemma 42 for the comparisons specified in Proposition 46. The additional regularity of $\mathbf{b}$ and $\overline{\mathbf{b}}$ does not have any influence on the inequalities though. Indeed, for each $h \in[0,1]$ and $\mu_{0} \in \mathcal{M}\left(\mathbb{R}^{N}\right)$, the map $(\mathbf{b}, c) \mapsto \vartheta_{\mathbf{b}, c}^{\mu}\left(h, \mu_{0}\right)$ with

$$
\begin{aligned}
& \vartheta_{\cdot, \cdot}^{\mu}\left(h, \mu_{0}\right):\left(W^{1, \infty}\left(\mathbb{R}^{N}, \mathbb{R}^{N}\right) \cap C^{1},\|\cdot\|_{L^{\infty}}\right) \times\left(W^{1, \infty}\left(\mathbb{R}^{N}, \mathbb{R}\right),\|\cdot\|_{L^{\infty}}\right) \\
& \quad \rightarrow\left(\mathcal{M}\left(\mathbb{R}^{N}\right), d_{\mathcal{M}}\right)
\end{aligned}
$$


is continuous according to statement (6). For this reason, we can extend all statements in Proposition 46 to arbitrary $\mathbf{b}, \overline{\mathbf{b}} \in W^{1, \infty}\left(\mathbb{R}^{N}, \mathbb{R}^{N}\right)$ because $C^{1}\left(\mathbb{R}^{N}, \mathbb{R}^{N}\right) \cap$ $W^{1, \infty}\left(\mathbb{R}^{N}, \mathbb{R}^{N}\right)$ is dense in $W^{1, \infty}\left(\mathbb{R}^{N}, \mathbb{R}^{N}\right)$ with respect to the $L^{\infty}$ norm and, bounded subsets of $\mathcal{M}\left(\mathbb{R}^{N}\right)$ are complete w.r.t. $d_{\mathcal{M}}$ as specified in condition (3) of Proposition 40 .

Proof of Proposition 46 Statements (1) and (2) follow directly from the explicit formula in Proposition 43 and the semi-group property of the flow $\mathfrak{X}_{1, \mathbf{b}}(\cdot, \cdot)$, i.e., $\mathfrak{X}_{1, \mathbf{b}}\left(h, \mathfrak{X}_{1, \mathbf{b}}(t, \mathbf{x})\right)=\mathfrak{X}_{1, \mathbf{b}}(t+h, \mathbf{x})$ for all $\mathbf{x} \in \mathbb{R}^{N}$ and $t, h \geq 0$.

(3) The total variation of any measure $\mu \in \mathcal{M}\left(\mathbb{R}^{N}\right)$ in an open set $A \subset \mathbb{R}^{N}$ is

$$
|\mu|(A)=\sup \left\{\int_{\mathbb{R}^{N}} \varphi d \mu \mid \varphi \in C_{c}^{0}(A),\|\varphi\|_{L^{\infty}} \leq 1\right\}
$$

due to [7, Proposition 1.47]. Thus, we conclude from Proposition 43 for every $\mu_{0} \in$ $\mathcal{M}\left(\mathbb{R}^{N}\right)$ and $h \in[0,1]$

$$
\begin{aligned}
& \left|\vartheta_{\mathbf{b}, c}^{\mu}\left(h, \mu_{0}\right)\right|\left(\mathbb{R}^{N}\right) \\
& \quad=\sup \left\{\int_{\mathbb{R}^{N}} \varphi \vartheta_{\mathbf{b}, c}^{\mu}\left(h, \mu_{0}\right) \mid \varphi \in C_{c}^{0}\left(\mathbb{R}^{N}\right),\|\varphi\|_{L^{\infty}} \leq 1\right\} \\
& \quad=\sup \left\{\int_{\mathbb{R}^{N}} \varphi\left(\mathfrak{X}_{1, \mathbf{b}}(t, \mathbf{x})\right) \cdot e^{\int_{0}^{h} c\left(\mathfrak{X}_{1, \mathbf{b}}(s, \mathbf{x})\right) d s} d \mu_{0}(\mathbf{x}) \mid \varphi \in C_{c}^{0}\left(\mathbb{R}^{N}\right),\|\varphi\|_{L^{\infty}} \leq 1\right\} \\
& \quad \leq e^{\|c\|_{L^{\infty} h}} \cdot \sup \left\{\int_{\mathbb{R}^{N}}\left|\varphi\left(\mathfrak{X}_{1, \mathbf{b}}(t, \mathbf{x})\right)\right| d\left|\mu_{0}\right|(\mathbf{x}) \mid \varphi \in C_{c}^{0}\left(\mathbb{R}^{N}\right),\|\varphi\|_{\left.L^{\infty} \leq 1\right\}}\right. \\
& \leq e^{\|c\|_{L^{\infty} h}} \cdot\left|\mu_{0}\right|\left(\mathbb{R}^{N}\right) .
\end{aligned}
$$

(4) Let $\varphi \in C_{c}^{\infty}\left(\mathbb{R}^{N}\right)$ be an arbitrary function with $\|\varphi\|_{L^{\infty}} \leq 1$ and $\|\nabla \varphi\|_{L^{\infty}} \leq 1$. Due to Proposition 43 again, we obtain for every $\mu_{0} \in \mathcal{M}\left(\mathbb{R}^{N}\right)$ and $t, h \in[0,1]$ with $t+h \leq 1$

$$
\begin{aligned}
& \int_{\mathbb{R}^{N}} \varphi d\left(\vartheta_{\mathbf{b}, c}^{\mu}\left(t+h, \mu_{0}\right)-\vartheta_{\mathbf{b}, c}^{\mu}\left(t, \mu_{0}\right)\right) \\
& =\int_{t}^{t+h} \frac{d}{d s} \int_{\mathbb{R}^{N}} \varphi(\mathbf{x}) d \vartheta_{\mathbf{b}, c}^{\mu}\left(s, \mu_{0}\right)(\mathbf{x}) d s \\
& =\int_{t}^{t+h} \int_{\mathbb{R}^{N}}(\nabla \varphi(\mathbf{x}) \cdot \mathbf{b}(\mathbf{x})+\varphi(\mathbf{x}) c(\mathbf{x})) d \vartheta_{\mathbf{b}, c}^{\mu}\left(s, \mu_{0}\right)(\mathbf{x}) d s \\
& \leq \int_{t}^{t+h}\left(\|\nabla \varphi\|_{L^{\infty}}\|\mathbf{b}\|_{L^{\infty}}+\|\varphi\|_{L^{\infty}}\|c\|_{L^{\infty}}\right)\left|\vartheta_{\mathbf{b}, c}^{\mu}\left(s, \mu_{0}\right)\right|\left(\mathbb{R}^{N}\right) d s \\
& \leq h \cdot\left(\|\mathbf{b}\|_{L^{\infty}}+\|c\|_{L^{\infty}}\right) e^{\|c\|_{L^{\infty}}\left|\mu_{0}\right|\left(\mathbb{R}^{N}\right)}
\end{aligned}
$$

as a consequence of statement (3). The supremum with respect to all these functions $\varphi$ leads to claim (4) about $d_{\mathcal{M}}\left(\vartheta_{\mathbf{b}, c}^{\mu}\left(t, \mu_{0}\right), \vartheta_{\mathbf{b}, c}^{\mu}\left(t+h, \mu_{0}\right)\right)$. 
(5) Let $\varphi \in C_{c}^{\infty}\left(\mathbb{R}^{N}\right)$ again denote any function with $\|\varphi\|_{L^{\infty}} \leq 1$ and $\|\nabla \varphi\|_{L^{\infty}} \leq 1$. Then, any measures $\mu_{0}, v_{0} \in \mathcal{M}\left(\mathbb{R}^{N}\right)$ satisfy at every time $h \in[0,1]$

$$
\begin{aligned}
& \int_{\mathbb{R}^{N}} \varphi d\left(\vartheta_{\mathbf{b}, c}^{\mu}\left(h, \mu_{0}\right)-\vartheta_{\mathbf{b}, c}^{\mu}\left(h, v_{0}\right)\right) \\
& \quad=\int_{\mathbb{R}^{N}} \varphi\left(\mathfrak{X}_{1, \mathbf{b}}(h, \mathbf{x})\right) \cdot \exp \left(\int_{0}^{h} c\left(\mathfrak{X}_{1, \mathbf{b}}(s, \mathbf{x})\right) d s\right) d\left(\mu_{0}-v_{0}\right)(\mathbf{x}) \\
& \leq e^{\left(\operatorname{Lip} \mathbf{b}+\|c\|_{W^{1, \infty}}\right) h} \cdot d_{\mathcal{M}}\left(\mu_{0}, v_{0}\right) .
\end{aligned}
$$

Indeed, the last estimate results from assertion (1) of Proposition 40 because the composition

$$
\psi_{h}: \mathbb{R}^{N} \rightarrow \mathbb{R}^{N}, \quad \mathbf{x} \mapsto \varphi\left(\mathfrak{X}_{1, \mathbf{b}}(h, \mathbf{x})\right) \cdot \exp \left(\int_{0}^{h} c\left(\mathfrak{X}_{1, \mathbf{b}}(s, \mathbf{x})\right) d s\right)
$$

is continuously differentiable with compact support and, Lemma 42 implies that

$$
\left\|\psi_{h}\right\|_{L^{\infty}} \leq\|\varphi\|_{L^{\infty}} e^{\|c\|_{L^{\infty} h}} \leq e^{\|c\|_{L^{\infty} h}}
$$

and

$$
\begin{aligned}
& \left\|\nabla \psi_{h}\right\|_{L^{\infty}} \\
& \leq e^{\|c\|_{L^{\infty}} h}\left(\|\nabla \varphi\|_{L^{\infty}}\left\|\partial_{\mathbf{x}} \mathfrak{X}_{1, \mathbf{b}}(h, \cdot)\right\|_{L^{\infty}}+\|\varphi\|_{L^{\infty}} \cdot \int_{0}^{h}\|\nabla c\|_{L^{\infty}}\left\|\partial_{\mathbf{x}} \mathfrak{X}_{1, \mathbf{b}}(s, \cdot)\right\|_{L^{\infty}} d s\right) \\
& \leq e^{\|c\|_{L^{\infty} h}}\left(e^{\operatorname{Lipb} \cdot h}+h\|\nabla c\|_{L^{\infty}} e^{\operatorname{Lip} \mathbf{b} \cdot h}\right) \\
& \leq e^{\left(\operatorname{Lip} \mathbf{b}+\|c\|_{\left.L^{\infty}\right) h}\right.}\left(1+h\|\nabla c\|_{L^{\infty}}\right) \\
& \leq e^{\left(\operatorname{Lip} \mathbf{b}+\|c\|_{\left.L^{\infty}\right) h}\right.} e^{h\|\nabla c\|_{L^{\infty}}}=e^{\left(\operatorname{Lip} \mathbf{b}+\|c\|_{W^{1, \infty}}\right) h} .
\end{aligned}
$$

The supremum with respect to all $\varphi \in C_{c}^{\infty}\left(\mathbb{R}^{N}\right)$ satisfying $\|\varphi\|_{L^{\infty}} \leq 1$ and $\|\nabla \varphi\|_{L^{\infty}} \leq$ 1 leads to

$$
d_{\mathcal{M}}\left(\vartheta_{\mathbf{b}, c}^{\mu}\left(h, \mu_{0}\right), \vartheta_{\mathbf{b}, c}^{\mu}\left(h, v_{0}\right)\right) \leq e^{\left(\operatorname{Lip} \mathbf{b}+\|c\|_{W^{1, \infty}}\right) h} \cdot d_{\mathcal{M}}\left(\mu_{0}, v_{0}\right) .
$$

(6) For estimating $d_{\mathcal{M}}\left(\vartheta_{\mathbf{b}, c}^{\mu}\left(h, \mu_{0}\right), \vartheta_{\overline{\mathbf{b}}, \bar{c}}^{\mu}\left(h, \mu_{0}\right)\right)$ with any $\mu_{0} \in \mathcal{M}\left(\mathbb{R}^{N}\right)$ and $h \in[0,1]$, we again choose an arbitrary function $\varphi \in C_{c}^{\infty}\left(\mathbb{R}^{N}\right)$ with $\|\varphi\|_{L^{\infty}} \leq 1$ and $\|\nabla \varphi\|_{L^{\infty}} \leq 1$ and consider now an appropriate convex combination $\psi:[0,1] \times$ $[0,1] \times \mathbb{R}^{N} \rightarrow \mathbb{R}^{N}$ :

$$
\begin{aligned}
\psi(\lambda, h, \mathbf{x}):= & \varphi\left(\lambda \mathfrak{X}_{1, \mathbf{b}}(h, \mathbf{x})+(1-\lambda) \mathfrak{X}_{1, \overline{\mathbf{b}}}(h, \mathbf{x})\right) \\
& \cdot e^{\int_{0}^{h} \lambda \cdot c\left(\mathfrak{X}_{1, \mathbf{b}}(r, \mathbf{x})\right)+(1-\lambda) \cdot \bar{c}\left(\mathfrak{X}_{1, \overline{\mathbf{b}}}(r, \mathbf{x})\right) d r} .
\end{aligned}
$$


Obviously, $\psi$ is continuously differentiable and, Lemma 42 ensures

$$
\begin{aligned}
& \left\|\frac{\partial}{\partial \lambda} \psi(\lambda, h, \cdot)\right\|_{L^{\infty}} \leq\|\nabla \varphi\|_{L^{\infty}}\left\|\mathfrak{X}_{1, \mathbf{b}}(h, \cdot)-\mathfrak{X}_{1, \overline{\mathbf{b}}}(h, \cdot)\right\|_{L^{\infty}} \cdot e^{h \cdot \max \left\{\|c\|_{\left.L^{\infty},\|\bar{c}\|_{L^{\infty}}\right\}}\right.} \\
& +\|\varphi\|_{L^{\infty}} \cdot \int_{0}^{h}\left\|c\left(\mathfrak{X}_{1, \mathbf{b}}(r, \cdot)\right)-\bar{c}\left(\mathfrak{X}_{1, \overline{\mathbf{b}}}(r, \cdot)\right)\right\|_{L^{\infty}} d r \\
& \times e^{h \cdot \max \left\{\|c\|_{\left.L^{\infty},\|\bar{c}\|_{L} \infty\right\}}\right.} \\
& \leq\|\mathbf{b}-\overline{\mathbf{b}}\|_{L^{\infty}} h e^{h \operatorname{Lip} \mathbf{b}} \cdot e^{h \cdot \max \left\{\|c\|_{L},\|\bar{c}\|_{L} \infty\right\}} \\
& +h\left(\|c-\bar{c}\|_{L^{\infty}}+\|\nabla c\|_{L^{\infty}}\|\mathbf{b}-\overline{\mathbf{b}}\|_{L^{\infty}} h e^{h \cdot \operatorname{Lip} \mathbf{b}}\right) \\
& \cdot e^{h \cdot \max \left\{\|c\|_{L} \infty,\|\bar{c}\|_{L^{\infty}}\right\}} \\
& \leq\left(\|\mathbf{b}-\overline{\mathbf{b}}\|_{L^{\infty}} e^{h\|\nabla c\|_{L^{\infty}}}+\|c-\bar{c}\|_{L^{\infty}}\right) h \\
& \times e^{h \cdot\left(\operatorname{Lip} \mathbf{b}+\max \left\{\|c\|_{L} \infty,\|\bar{c}\|_{L^{\infty}}\right\}\right)} .
\end{aligned}
$$

Hence we obtain

$$
\begin{aligned}
& \int_{\mathbb{R}^{N}} \varphi d\left(\vartheta_{\mathbf{b}, c}^{\mu}\left(h, \mu_{0}\right)-\vartheta_{\overline{\mathbf{b}}, \bar{c}}^{\mu}\left(h, \mu_{0}\right)\right)=\int_{\mathbb{R}^{N}}(\psi(1, h, \mathbf{x})-\psi(0, h, \mathbf{x})) d \mu_{0}(\mathbf{x}) \\
& =\int_{\mathbb{R}^{N}} \int_{0}^{1} \frac{\partial}{\partial \lambda} \psi(\lambda, h, \mathbf{x}) d \lambda d \mu_{0}(\mathbf{x}) \\
& \leq\left\|\frac{\partial}{\partial \lambda} \psi(\lambda, h, \cdot)\right\|_{L^{\infty}}\left|\mu_{0}\right|\left(\mathbb{R}^{N}\right) \\
& \leq\left(\|\mathbf{b}-\overline{\mathbf{b}}\|_{L^{\infty}} e^{h\|\nabla c\|_{L^{\infty}}}+\|c-\bar{c}\|_{L^{\infty}}\right) h \\
& \times e^{h \cdot\left(\operatorname{Lipb}+\max \left\{\|c\|_{L} \infty,\|\bar{c}\|_{L^{\infty}}\right\}\right)}\left|\mu_{0}\right|\left(\mathbb{R}^{N}\right) .
\end{aligned}
$$

\section{Euler compactness method for measure-valued solutions}

Similarly to Proposition 32 about $L^{p}\left(\mathbb{R}^{N}\right)$-valued solutions, we now specify the special form of sequential compactness that is sufficient for concluding the existence of solutions (to the full nonlinear problem) from Euler approximations (on the basis of autonomous linear problems).

Proposition 47 Choose $\mu_{0} \in \mathcal{M}\left(\mathbb{R}^{N}\right), T>0$ and $R>0$ arbitrarily. Let $\mathcal{N}_{\mathcal{M}}=$ $\mathcal{N}_{\mathcal{M}}\left(\mu_{0}, T, R\right)$ denote the subset of all curves $\mu:[0, T] \rightarrow \mathcal{M}\left(\mathbb{R}^{N}\right)$ constructed in the following piecewise way: For an arbitrary equidistant partition $0=t_{0}<t_{1}<$ $\cdots<t_{n}=T$ of $[0, T]$ (with $n>T$ ) and functions $\mathbf{b}_{1}, \ldots, \mathbf{b}_{n} \in W^{1, \infty}\left(\mathbb{R}^{N}, \mathbb{R}^{N}\right.$ ) and $c_{1}, \ldots, c_{n} \in W^{1, \infty}\left(\mathbb{R}^{N}, \mathbb{R}\right)$ with $\max \left\{\left\|\mathbf{b}_{k}\right\|_{W^{1, \infty}},\left\|c_{k}\right\|_{W^{1, \infty}} \mid 1 \leq k \leq n\right\} \leq R$, define $\mu:[0, T] \rightarrow \mathcal{M}\left(\mathbb{R}^{N}\right)$ as

$$
\mu_{t}:=\vartheta_{\mathbf{b}_{k}, c_{k}}^{\mu}\left(t-t_{k-1}, \mu_{t_{k-1}}\right) \quad \text { for } t \in\left(t_{k-1}, t_{k}\right], \quad k=1,2, \ldots, n
$$


Then at each time $t \in[0, T]$, the set $\left\{\mu_{t} \mid \mu(\cdot) \in \mathcal{N}_{\mathcal{M}}\right\} \subset \mathcal{M}\left(\mathbb{R}^{N}\right)$ is relatively compact with respect to the $W^{1, \infty}$ dual metric $d_{\mathcal{M}}$. Furthermore, the set of all measure values of $\mathcal{N}_{\mathcal{M}}\left(\mu_{0}, T, R\right)$, i.e., $\left\{\mu_{t} \mid t \in[0, T], \mu(\cdot) \in \mathcal{N}_{\mathcal{M}}\right\} \subset \mathcal{M}\left(\mathbb{R}^{N}\right)$, is tight.

Proof As a piecewise consequence of assertion (3) of Proposition 46 the total variation $|\nu|\left(\mathbb{R}^{N}\right)$ is uniformly bounded for all measures $v \in\left\{\mu_{t} \mid t \in[0, T], \mu(\cdot) \in\right.$ $\left.\mathcal{N}_{\mathcal{M}}\right\} \subset \mathcal{M}\left(\mathbb{R}^{N}\right):|v|\left(\mathbb{R}^{N}\right) \leq e^{R T}\left|\mu_{0}\right|\left(\mathbb{R}^{N}\right)$. It suffices to prove that this set $\left\{\mu_{t} \mid t \in[0, T], \mu(\cdot) \in \mathcal{N}_{\mathcal{M}}\right\} \subset \mathcal{M}\left(\mathbb{R}^{N}\right)$ is tight. Indeed, assertion (4) of Proposition 40 then implies its relative compactness w.r.t. the metric $d_{\mathcal{M}}$.

For every $\varepsilon>0$, there exists a compact subset $K_{\varepsilon} \subset \mathbb{R}^{N}$ with $\left|\mu_{0}\right|\left(\mathbb{R}^{N} \backslash K_{\varepsilon}\right)<\varepsilon$. We conclude

$$
\left|\mu_{t}\right|\left(\mathbb{R}^{N} \backslash \mathbb{B}_{R T}\left(K_{\varepsilon}\right)\right) \leq\left|\mu_{t}\right|\left(\mathbb{R}^{N} \backslash \mathbb{B}_{R t}\left(K_{\varepsilon}\right)\right)<\varepsilon e^{R t} \leq \varepsilon e^{R T}
$$

for all $t \in[0, T]$ and $\mu(\cdot) \in \mathcal{N}_{\mathcal{M}}\left(\mu_{0}, T, R\right)$. Indeed, let $0=t_{0}<t_{1}<\ldots<$ $t_{n}=T$ denote the underlying equidistant partition of $[0, T]$ and with $\mathbf{b}_{1}, \ldots, \mathbf{b}_{n} \in$ $W^{1, \infty}\left(\mathbb{R}^{N}, \mathbb{R}^{N}\right)$ and $c_{1}, \ldots, c_{n} \in W^{1, \infty}\left(\mathbb{R}^{N}, \mathbb{R}\right)$ denote

$$
\mu_{t}=\vartheta_{\mathbf{b}_{k}, c_{k}}^{\mu}\left(t-t_{k-1}, \mu_{t_{k-1}}\right) \quad \text { for } t \in\left(t_{k-1}, t_{k}\right], \quad k=1,2, \ldots n .
$$

From Proposition 43, we obtain for each $t \in\left(t_{k-1}, t_{k}\right]$ by induction with respect to $k$

$$
\begin{aligned}
\left|\mu_{t}\right|\left(\mathbb{R}^{N} \backslash \mathbb{B}_{R t}\left(K_{\varepsilon}\right)\right) & =\sup \left\{\int_{\mathbb{R}^{N}} \varphi d \vartheta_{\mathbf{b}_{k}, c_{k}}^{\mu}\left(t-t_{k-1}, \mu_{t_{k-1}}\right) \mid \varphi \in C_{c}^{0}\left(\mathbb{R}^{N} \backslash \mathbb{B}_{R t}\left(K_{\varepsilon}\right)\right),\|\varphi\|_{\left.L^{\infty} \leq 1\right\}}\right. \\
\leq & \sup \left\{\int_{\mathbb{R}^{N}} \bar{\varphi}\left(\mathfrak{X}_{1, \mathbf{b}_{k}}\left(t-t_{k-1}, \mathbf{x}\right)\right) d \mu_{t_{k-1}}(\mathbf{x}) \cdot e^{\left(t-t_{k-1}\right) R} \mid \bar{\varphi} \in C_{c}^{0}\left(\mathbb{R}^{N} \backslash \mathbb{B}_{R t}\left(K_{\varepsilon}\right)\right),\right. \\
& \left.\|\bar{\varphi}\|_{L^{\infty} \leq 1}\right\} \\
\leq & \sup \left\{\int_{\mathbb{R}^{N}} \psi(\mathbf{x}) d \mu_{t_{k-1}}(\mathbf{x}) \cdot e^{\left(t-t_{k-1}\right) R} \mid \psi \in C_{c}^{0}\left(\mathbb{R}^{N} \backslash \mathbb{B}_{R t_{k-1}}\left(K_{\varepsilon}\right)\right),\|\psi\|_{L^{\infty} \leq 1}\right\} \\
= & e^{\left(t-t_{k-1}\right) R}\left|\mu_{t_{k-1}}\right|\left(\mathbb{R}^{N} \backslash \mathbb{B}_{R t_{k-1}}\left(K_{\varepsilon}\right)\right) .
\end{aligned}
$$

Corollary 48 The closure of $\mathcal{N}_{\mathcal{M}}\left(\mu_{0}, T, R\right) \subset \mathcal{M}\left(\mathbb{R}^{N}\right)$ with respect to $d_{\mathcal{M}}$ is tight.

Proof Consider any Radon measure $\zeta$ in the closure of $\mathcal{N}_{\mathcal{M}}\left(\mu_{0}, T, R\right)$ w.r.t. $d_{L^{p}}$, i.e., there is a sequence $\left(\zeta_{k}\right)_{k \in \mathbb{N}}$ with $d_{\mathcal{M}}\left(\zeta_{k}, \zeta\right) \rightarrow 0$ for $k \rightarrow \infty$. This implies $\zeta_{k} \rightarrow \zeta$ weakly* in $\mathcal{M}\left(\mathbb{R}^{N}\right)=C_{0}^{0}\left(\mathbb{R}^{N}\right)^{\prime}$ due to assertion (2) of Proposition 40 .

For $\varepsilon>0$ fixed arbitrarily, Proposition 47 provides a compact subset $K_{\varepsilon} \subset \mathbb{R}^{N}$ with $|\eta|\left(\mathbb{R}^{N} \backslash K_{\varepsilon}\right)<\varepsilon$ for all $\eta \in \mathcal{N}_{\mathcal{M}}\left(\mu_{0}, T, R\right)$. Considering just the restrictions to $\mathbb{R}^{N} \backslash K_{\varepsilon}$, we conclude from $\zeta_{k} \rightarrow \zeta$ weakly* in $\mathcal{M}\left(\mathbb{R}^{N} \backslash K_{\varepsilon}\right)=C_{0}^{0}\left(\mathbb{R}^{N} \backslash K_{\varepsilon}\right)^{\prime}$ that $|\zeta|\left(\mathbb{R}^{N} \backslash K_{\varepsilon}\right) \leq \liminf _{k \rightarrow \infty}\left|\zeta_{k}\right|\left(\mathbb{R}^{N} \backslash K_{\varepsilon}\right)<\varepsilon$ (see, e.g., [68, § V.1 Theorem 9 (ii)]). Hence the closure of $\mathcal{N}_{\mathcal{M}}\left(\mu_{0}, T, R\right) \subset \mathcal{M}\left(\mathbb{R}^{N}\right)$ w.r.t. $d_{\mathcal{M}}$ is tight. 


\subsection{Proofs on the well-posedness of the nonlocal balance law in Sect. 3.2}

We follow similar arguments to those presented for $L^{p}\left(\mathbb{R}^{N}\right)$-valued solutions in Sect. 5.1 to the results stated in Sect. 3.2.

An infinitesimal condition w.r.t. $d_{\mathcal{M}}$ lays the foundations for another type of solutions whose existence can be verified by means of Euler compactness method. This alternative criterion represents what is usually considered in mutational equations in the (purely) metric setting (see $[10,11,51]$ ). Then we provide a connection between these generalised ("mutational") solutions and distributional solutions to the transport equation.

Proposition 49 Consider the coefficient functions $\mathcal{B}:[0, T] \times \mathcal{M}\left(\mathbb{R}^{N}\right) \rightarrow$ $W^{1, \infty}\left(\mathbb{R}^{N}, \mathbb{R}^{N}\right), \mathcal{C}:[0, T] \times \mathcal{M}\left(\mathbb{R}^{N}\right) \rightarrow W^{1, \infty}\left(\mathbb{R}^{N}, \mathbb{R}\right)$ under the assumptions (i) and (ii) of Theorem 7 and the hypothesis

$$
\left(i v^{\prime}\right) \mathcal{B}, \mathcal{C}:[0, T] \times\left(\mathcal{M}\left(\mathbb{R}^{N}\right), d_{\mathcal{M}}\right) \rightarrow\left(L^{\infty},\|\cdot\|_{L^{\infty}}\right) \text { are continuous. }
$$

Then there exists a function $\mu:[0, T] \rightarrow \mathcal{M}\left(\mathbb{R}^{N}\right)$ with the following properties:

(1) $\mu$ is bounded with respect to the total variation and, $\left\{\mu_{t} \mid t \in[0, T]\right\} \subset \mathcal{M}\left(\mathbb{R}^{N}\right)$ is tight.

(2) $\mu:[0, T] \rightarrow\left(\mathcal{M}\left(\mathbb{R}^{N}\right), d_{\mathcal{M}}\right)$ is continuous,

(3) $\lim _{h \downarrow 0} \frac{1}{h} \cdot d_{\mathcal{M}}\left(\mu_{t+h}, \vartheta_{\left(\mathcal{B}\left(t, \mu_{t}\right), \mathcal{C}\left(t, \mu_{t}\right)\right)}^{\mu}\left(h, \mu_{t}\right)\right)=0$ for a.e. $t \in[0, T[$,

(4) $\mu:[0, T] \rightarrow \mathcal{M}\left(\mathbb{R}^{N}\right)$ is a narrowly continuous distributional solution to initial value problem (3).

This existence result can be extended to Theorem 7 by means of the Scorza-Dragoni theorem in metric spaces (i.e., Lemma 37). The corresponding arguments of approximation are the same as from Proposition 34 to Theorem 4 (presented in Sect. 5.1).

The proof of Proposition 49 consists of several lemmata.

Lemma 50 (A "mutational" solution) Under the assumptions of Proposition 49, there exists a function $\mu:[0, T] \rightarrow \mathcal{M}\left(\mathbb{R}^{N}\right)$ with properties (1)-(3) specified there.

Proof (Sketch) The shortest formulation might be: We can simply apply [51, Theorem 2.18] to the tuple $\left(\mathcal{M}\left(\mathbb{R}^{N}\right), d_{\mathcal{M}},|\cdot|\left(\mathbb{R}^{N}\right)\right)$. For the sake of a self-contained presentation, we summarize the gist of the proof as follows.

The steps for verifying Lemma 35 are all based on the Euler method in combination with the suitable form of compactness (presented in Proposition 47). A subsequence of Euler approximations proves to converge to a Lipschitz continuous function $\mu:[0, T] \rightarrow\left(\mathcal{M}\left(\mathbb{R}^{N}\right), d_{\mathcal{M}}\right)$ pointwise w.r.t. $d_{\mathcal{M}}$. Corollary 48 guarantees that $\left\{\mu_{t} \mid t \in[0, T]\right\} \subset \mathcal{M}\left(\mathbb{R}^{N}\right)$ is tight. The estimates for the distances between two Euler approximations result from Proposition 46 in a piecewise way if we take the following a priori bounds into consideration:

$$
\eta:=\sup _{t, \zeta}\left(\|\mathcal{B}(t, \zeta)\|_{L^{\infty}}+\|\mathcal{C}(t, \zeta)\|_{L^{\infty}}\right) \stackrel{(\mathrm{i})}{<} \infty, \quad \rho:=\left|\mu_{0}\right|\left(\mathbb{R}^{N}\right) \cdot e^{\eta T}+1
$$

and the constant $C_{\rho}$ according to hypothesis (ii) of Theorem 7. 
Lemma 51 (A distributional solution) Under the assumptions of Proposition 49, every function $\mu:[0, T] \rightarrow \mathcal{M}\left(\mathbb{R}^{N}\right)$ with properties (1)-(3) is narrowly continuous and solves

$$
\partial_{t} \mu+\operatorname{div}_{\mathbf{x}}(\mathcal{B}(t, \mu) \mu)=\mathcal{C}(t, \mu) \cdot \mu
$$

in the distributional sense, i.e., for any $0 \leq t_{1}<t_{2} \leq T, \varphi \in C_{c}^{1}\left(\mathbb{R}^{N}\right)$,

$$
\begin{aligned}
\int_{\mathbb{R}^{N}} \varphi d \mu_{t_{2}}-\int_{\mathbb{R}^{N}} \varphi d \mu_{t_{1}}= & \int_{t_{1}}^{t_{2}} \int_{\mathbb{R}^{N}} \mathcal{B}\left(s, \mu_{s}\right)(\mathbf{x}) \cdot \nabla_{\mathbf{x}} \varphi(\mathbf{x}) d \mu_{s}(\mathbf{x}) d s \\
& +\int_{t_{1}}^{t_{2}} \int_{\mathbb{R}^{N}} \mathcal{C}\left(s, \mu_{s}\right)(\mathbf{x}) \varphi(\mathbf{x}) d \mu_{s}(\mathbf{x}) d s .
\end{aligned}
$$

Proof The continuity w.r.t. $d_{\mathcal{M}}$ and tight values always imply the narrow continuity due to Proposition 40 (2). Moreover, for every $\varphi \in C_{c}^{1}\left(\mathbb{R}^{N}\right)$, the auxiliary function $\psi:[0, T] \rightarrow \mathbb{R}$ defined by $\psi(t)=\int_{\mathbb{R}^{N}} \varphi(\mathbf{x}) d \mu_{t}(\mathbf{x})$ is continuous and satisfies for Lebesgue-almost every $t \in[0, T)$

$$
\begin{aligned}
\lim _{h \downarrow 0} & \frac{1}{h} \cdot(\psi(t+h)-\psi(t)) \\
= & \lim _{h \downarrow 0} \frac{1}{h} \cdot\left(\int_{\mathbb{R}^{N}} \varphi d \mu_{t+h}-\int_{\mathbb{R}^{N}} \varphi d \mu_{t}\right) \\
= & \lim _{h \downarrow 0} \frac{1}{h} \cdot\left(\int_{\mathbb{R}^{N}} \varphi d \mu_{t+h}-\int_{\mathbb{R}^{N}} \varphi d \vartheta_{\left(\mathcal{B}\left(t, \mu_{t}\right), \mathcal{C}\left(t, \mu_{t}\right)\right)}^{\mu}\left(h, \mu_{t}\right)\right) \\
& +\lim _{h \downarrow 0} \frac{1}{h} \cdot\left(\int_{\mathbb{R}^{N}} \varphi d \vartheta_{\left(\mathcal{B}\left(t, \mu_{t}\right), \mathcal{C}\left(t, \mu_{t}\right)\right)}^{\mu}\left(h, \mu_{t}\right)-\int_{\mathbb{R}^{N}} \varphi d \mu_{t}\right) \\
= & 0+\lim _{h \downarrow 0} \frac{1}{h} \cdot\left(\int_{\mathbb{R}^{N}} \varphi\left(\mathfrak{X}_{1, \mathcal{B}\left(t, \mu_{t}\right)}(h, \mathbf{x})\right) \cdot e^{\int_{0}^{h} \mathcal{C}\left(t, \mu_{t}\right)\left(\mathfrak{X}_{1, \mathcal{B}\left(t, \mu_{t}\right)}(s, \mathbf{x})\right) d s} d \mu_{t}(\mathbf{x})\right. \\
& \left.-\int_{\mathbb{R}^{N}} \varphi d \mu_{t}\right) \\
= & \int_{\mathbb{R}^{N}}\left(\nabla \varphi(\mathbf{y}) \cdot \mathcal{B}\left(t, \mu_{t}\right)(\mathbf{y})+\varphi(\mathbf{y}) \mathcal{C}\left(t, \mu_{t}\right)(\mathbf{y})\right) d \mu_{t}(\mathbf{y})
\end{aligned}
$$

due to the property (3) of $\mu(\cdot)$ and Proposition 43 respectively.

These two lemmata imply Proposition 49, i.e., the main result about the existence of a measure-valued solution to nonlocal balance law (3) whenever both coefficients $\mathcal{B}, \mathcal{C}:[0, T] \times\left(\mathcal{M}\left(\mathbb{R}^{N}\right), d_{\mathcal{M}}\right) \rightarrow\left(L^{\infty},\|\cdot\|_{L^{\infty}}\right)$ are assumed to be continuous (in addition). This statement can be extended to Carathéodory coefficient functions by means of Scorza-Dragoni theorem in metric spaces (i.e., Lemma 37) and so, we finally obtain Theorem 7.

In the next step, we provide a connection from narrowly continuous distributional solutions to the generalised type of solution described in Lemma 50. The following statement plays the role of Lemma 38 in the setting of measure-valued distributional 
solutions. The narrow topology implies the analytical advantage that the number of assumptions is smaller this time since some features (of distributional solutions) can be concluded.

Lemma 52 Under the assumptions of Theorem 7 , let $\mu:[0, T] \rightarrow \mathcal{M}\left(\mathbb{R}^{N}\right)$, satisfy the following conditions:

(1) $\mu$ is narrowly continuous,

(2) $\mu:[0, T] \rightarrow \mathcal{M}\left(\mathbb{R}^{N}\right)$ is a distributional solution to initial value problem (3) (in the sense of $E q$. (4) for any $0 \leq t_{1}<t_{2} \leq T$ and $\varphi \in C_{c}^{1}\left(\mathbb{R}^{N}\right)$ ).

Then, $\mu$ is bounded with respect to the total variation and, the set $\left\{\mu_{t} \mid t \in[0, T]\right\} \subset$ $\mathcal{M}\left(\mathbb{R}^{N}\right)$ is tight. Moreover, $\mu$ is continuous w.r.t. the metric $d_{\mathcal{M}}$ and, at Lebesgue almost every time instant $t \in[0, T)$ it satisfies the infinitesimal condition

$$
\lim _{h \downarrow 0} \frac{1}{h} \cdot d_{\mathcal{M}}\left(\mu_{t+h}, \vartheta_{\left(\mathcal{B}\left(t, \mu_{t}\right), \mathcal{C}\left(t, \mu_{t}\right)\right)}^{\mu}\left(h, \mu_{t}\right)\right)=0 .
$$

Proof $\mu:[0, T] \rightarrow\left(\mathcal{M}\left(\mathbb{R}^{N}\right)\right.$, narrow $)$ is assumed to be continuous and so, the image set $\mu([0, T]) \subset \mathcal{M}\left(\mathbb{R}^{N}\right)$ is compact with respect to the narrow topology. According to the generalisation of Prokhorov's theorem to Borel measures on a complete separable metric space in [15, Theorem 8.6.2], this compactness implies that $\mu([0, T]) \subset \mathcal{M}\left(\mathbb{R}^{N}\right)$ is both bounded w.r.t. the total variation and tight (in the sense of Definition 39). Hence, $\mu:[0, T] \rightarrow\left(\mathcal{M}\left(\mathbb{R}^{N}\right), d_{\mathcal{M}}\right)$ is continuous according to assertion (2) of Proposition 40.

The coefficients $\mathcal{B}, \mathcal{C}:[0, T] \times\left(\mathcal{M}\left(\mathbb{R}^{N}\right), d_{\mathcal{M}}\right) \rightarrow\left(L^{\infty},\|\cdot\|_{L^{\infty}}\right)$ are Carathéodory functions by assumptions (iii) and (iv) of Theorem 7 . Hence the composite coefficients

$$
\begin{aligned}
\widetilde{\mathbf{b}} & :=\mathcal{B}(\cdot, \mu(\cdot)):[0, T] \rightarrow\left(W^{1, \infty}\left(\mathbb{R}^{N}, \mathbb{R}^{N}\right),\|\cdot\|_{L^{\infty}}\right), \\
\widetilde{c} & :=\mathcal{C}(\cdot, \mu(\cdot)):[0, T] \rightarrow\left(W^{1, \infty}\left(\mathbb{R}^{N}\right),\|\cdot\|_{L^{\infty}}\right)
\end{aligned}
$$

are Lebesgue measurable and bounded. Proposition 49 provides a function $v$ : $[0, T] \rightarrow \mathcal{M}\left(\mathbb{R}^{N}\right)$ which is a narrowly continuous distributional solution to the nonautonomous linear problem

$$
\partial_{t} v+\operatorname{div}_{\mathbf{x}}(\widetilde{\mathbf{b}}(t) v)=\widetilde{c}(t) \cdot v \quad \text { in }[0, T], \quad v(0)=\mu_{0}
$$

and satisfies the infinitesimal condition at Lebesgue-almost every time instant $t \in$ $[0, T)$

$$
\lim _{h \downarrow 0} \frac{1}{h} \cdot d_{\mathcal{M}}\left(v_{t+h}, \vartheta_{(\widetilde{\mathbf{b}}(t), \widetilde{c}(t))}^{\mu}\left(h, v_{t}\right)\right)=0 .
$$

The comparison principle in Proposition 44 implies the uniqueness of solutions to (21) and so, $\nu$ and $\mu$ coincide in $[0, T]$. Thus, $\mu$ satisfies the claimed infinitesimal condition w.r.t. $d_{\mathcal{M}}$. 
This equivalence of solution criteria lays the basis for proving Proposition 8 (about uniqueness of distributional solutions) and Proposition 9 (about its continuous dependence of data) by the same arguments as for $L^{p}\left(\mathbb{R}^{N}\right)$-valued solutions in Sect. 5.1. In addition to Proposition 46, the key tool is Gronwall's inequality again (see [51, Proposition A.2]).

Acknowledgments Parts of these results were elaborated during the second author's stay at the Institute for Pure and Applied Mathematics (IPAM) at the University of California in Los Angeles (UCLA). T.L. thanks Prof. Alexandre Bayen, Prof. Christian Claudel, Prof. Daniel Work and co-organizers for the kind invitation to the long-term program on "New Directions in Mathematical Approaches for Traffic Flow Management" in autumn 2015. Furthermore he expresses his gratitude to Prof. Rinaldo M. Colombo and Prof. Martin Gugat for fruitful complementary discussions.

The first author was partially supported by the Chinese NSF Grant 11571125, "The Recruitment Program for Foreign Experts", China, and the Spanish Ministerio de Economía y Competitividad project MTM201563723-P

Open Access This article is distributed under the terms of the Creative Commons Attribution 4.0 International License (http://creativecommons.org/licenses/by/4.0/), which permits unrestricted use, distribution, and reproduction in any medium, provided you give appropriate credit to the original author(s) and the source, provide a link to the Creative Commons license, and indicate if changes were made.

\section{References}

1. Agarwal, R.P., O'Regan, D.: Existence for set differential equations via multivalued operator equations. In: Differential Equations and Applications, Vol. 5, pp. 1-5. Nova Sci. Publ., New York (2007)

2. Aggarwal, A., Colombo, R.M., Goatin, P.: Nonlocal systems of conservation laws in several space dimensions. SIAM J. Numer. Anal. 53(2), 963-983 (2015)

3. Alt, H.W.: Lineare Funktionalanalysis. Eine anwendungsorientierte Einführung, 6th revised edn. Springer, Berlin (2012)

4. Ambrosio, L.: Transport equation and Cauchy problem for $B V$ vector fields. Invent. Math. 158(2), 227-260 (2004)

5. Ambrosio, L.: Transport equation and Cauchy problem for non-smooth vector fields. In: Calculus of Variations and Nonlinear Partial Differential Equations, Lecture Notes in Math, vol. 1927, pp. 1-41. Springer, Berlin (2008)

6. Ambrosio, L., Bernard, P.: Uniqueness of signed measures solving the continuity equation for Osgood vector fields. Atti Accad. Naz. Lincei Cl. Sci. Fis. Mat. Natur. Rend. Lincei (9) Mat. Appl 19(3), 237-245 (2008)

7. Ambrosio, L., Fusco, N., Pallara, D.: Functions of Bounded Variation and Free Discontinuity Problems. Oxford Mathematical Monographs. The Clarendon Press, Oxford University Press, New York (2000)

8. Ambrosio, L., Gigli, N., Savaré, G.: Gradient Flows in Metric Spaces and in the Space of Probability Measures. Lectures in Mathematics ETH Zürich. Birkhäuser Verlag, Basel (2005)

9. Aubin, J.P.: A note on differential calculus in metric spaces and its applications to the evolution of tubes. Bull. Polish Acad. Sci. Math. 40(2), 151-162 (1992)

10. Aubin, J.P.: Mutational equations in metric spaces. Set-Valued Anal. 1(1), 3-46 (1993)

11. Aubin, J.P.: Mutational and Morphological Analysis. Systems and Control: Foundations and Applications. Tools for Shape Evolution and Morphogenesis. Birkhäuser Boston Inc., Boston, MA (1999)

12. Aubin, J.P.: Macroscopic traffic models: shifting from densities to "celerities". Appl. Math. Comput. 217(3), 963-971 (2010)

13. Aubin, J.P.: Regulation of viable and optimal cohorts. Appl. Math. Optim. 72(2), 203-232 (2015)

14. Aubin, J.P., Désilles, A.: Traffic Networks as Information Systems. Mathematical Engineering: A Viability Approach. Springer, Berlin (2017)

15. Bogachev, V.I.: Measure Theory, I, II edn. Springer, Berlin (2007)

16. Borsche, R., Colombo, R.M., Garavello, M., Meurer, A.: Differential equations modeling crowd interactions. J. Nonlinear Sci. 25(4), 827-859 (2015) 
17. Bouchut, F., Crippa, G.: Uniqueness, renormalization, and smooth approximations for linear transport equations. SIAM J. Math. Anal. 38(4), 1316-1328 (2006)

18. Bressan, A.: On the Cauchy problem for nonlinear hyperbolic systems. (Preprint ISAS-M-97-97) (1998)

19. Brezis, H.: Functional Analysis, Sobolev Spaces and Partial Differential Equations. Universitext. Springer, New York (2011)

20. Cañizo, J.A., Carrillo, J.A., Cuadrado, S.: Measure solutions for some models in population dynamics. Acta Appl. Math. 123, 141-156 (2013)

21. Carrillo, J.A., Colombo, R.M., Gwiazda, P., Ulikowska, A.: Structured populations, cell growth and measure valued balance laws. J. Differ. Equ. 252(4), 3245-3277 (2012)

22. Colombo, R.M., Corli, A., Rosini, M.D.: Non local balance laws in traffic models and crystal growth. ZAMM Z. Angew. Math. Mech. 87(6), 449-461 (2007)

23. Colombo, R.M., Garavello, M., Lécureux-Mercier, M.: A class of nonlocal models for pedestrian traffic. Math. Models Methods Appl. Sci. 22(4), 1150023, 34 (2012)

24. Colombo, R.M., Guerra, G.: Hyperbolic balance laws with a dissipative non local source. Commun. Pure Appl. Anal. 7(5), 1077-1090 (2008)

25. Colombo, R.M., Guerra, G.: Nonlocal sources in hyperbolic balance laws with applications. In: Hyperbolic Problems: Theory, Numerics, Applications, pp. 577-584. Springer, Berlin (2008)

26. Colombo, R.M., Guerra, G.: Balance laws as quasidifferential equations in metric spaces. In: Tadmor, A.E.T. , Liu, J.-G. (eds.) Hyperbolic Problems: Theory, Numerics and Applications, Proc. Sympos. Appl. Math., vol. 67, pp. 527-536. Amer. Math. Soc., Providence, RI (2009)

27. Colombo, R.M., Guerra, G.: Differential equations in metric spaces with applications. Discr. Contin. Dyn. Syst. 23(3), 733-753 (2009)

28. Colombo, R.M., Lécureux-Mercier, M.: Nonlocal crowd dynamics models for several populations. Acta Math. Sci. Ser. B Engl. Ed. 32(1), 177-196 (2012)

29. Colombo, R.M., Lorenz, T., Pogodaev, N.I.: On the modeling of moving populations through set evolution equations. Discr. Contin. Dyn. Syst. 35(1), 73-98 (2015)

30. Colombo, R.M., Marcellini, F.: Nonlocal systems of balance laws in several space dimensions with applications to laser technology. J. Differ. Equ. 259(11), 6749-6773 (2015)

31. Colombo, R.M., Pogodaev, N.: Confinement strategies in a model for the interaction between individuals and a continuum. SIAM J. Appl. Dyn. Syst. 11(2), 741-770 (2012)

32. Colombo, R.M., Pogodaev, N.: On the control of moving sets: positive and negative confinement results. SIAM J. Control Optim. 51(1), 380-401 (2013)

33. DiPerna, R.J., Lions, P.L.: Ordinary differential equations, transport theory and Sobolev spaces. Invent. Math. 98(3), 511-547 (1989)

34. Elstrodt, J.: Maß- und Integrationstheorie, 7th revised and updated edn. Springer-Lehrbuch. [Springer Textbook]. Springer, Berlin (2011). Grundwissen Mathematik. [Basic Knowledge in Mathematics]

35. Evans, L.C., Gariepy, R.F.: Measure Theory and Fine Properties of Functions. Studies in Advanced Mathematics. CRC Press, Boca Raton, FL (1992)

36. Goatin, P., Scialanga, S.: Well-posedness and finite volume approximations of the lwr traffic flow model with non-local velocity. Netw. Heterog. Media 11(1), 107-121 (2016)

37. Green, J.W., Valentine, F.A.: On the Arzelà-Ascoli theorem. Math. Mag. 34(4), 199-202 (1960/1961)

38. Gwiazda, P., Lorenz, T., Marciniak-Czochra, A.: A nonlinear structured population model: Lipschitz continuity of measure-valued solutions with respect to model ingredients. J. Differ. Equ. 248(11), 2703-2735 (2010)

39. Hartman, P.: Ordinary Differential Equations, Classics in Applied Mathematics, vol. 38. Society for Industrial and Applied Mathematics (SIAM), Philadelphia, PA (2002), corrected reprint of the second (1982) edition [Birkhäuser, Boston, MA; MR0658490 (83e:34002)], With a foreword by Peter Bates

40. Hirsch, M.W., Smale, S., Devaney, R.L.: Differential equations, dynamical systems, and an introduction to chaos. Pure and Applied Mathematics (Amsterdam), vol. 60, 2nd edn. Elsevier/Academic Press, Amsterdam (2004)

41. Kloeden, P.E., Lorenz, T.: Stochastic differential equations with nonlocal sample dependence. Stoch. Anal. Appl. 28(6), 937-945 (2010)

42. Kloeden, P.E., Lorenz, T.: Stochastic morphological evolution equations. J. Differ. Equ. 251(10), 29502979 (2011)

43. Kloeden, P.E., Lorenz, T.: A Peano-like theorem for stochastic differential equations with nonlocal sample dependence. Stoch. Anal. Appl. 31(1), 19-30 (2013) 
44. Kloeden, P.E., Lorenz, T.: A Peano theorem for fuzzy differential equations with evolving membership grade. Fuzzy Sets Syst. 280, 1-26 (2015)

45. Kloeden, P.E., Platen, E.: Numerical Solution of Stochastic Differential Equations. Applications of Mathematics (New York), vol. 23. Springer, Berlin (1992)

46. Lakshmikantham, V., Bhaskar, T.G., Vasundhara Devi, J.: Theory of Set Differential Equations in Metric Spaces. Cambridge Scientific Publishers, Cambridge (2006)

47. Lang, S.: Real and Functional Analysis. Graduate Texts in Mathematics, vol. 142, third edn. Springer, New York (1993)

48. Le Bris, C., Lions, P.L.: Renormalized solutions of some transport equations with partially $W^{1,1}$ velocities and applications. Ann. Mat. Pura Appl. (4) 183(1), 97-130 (2004)

49. Lorenz, T.: Set-valued maps for image segmentation. Comput. Vis. Sci. 4(1), 41-57 (2001)

50. Lorenz, T.: A viability theorem for morphological inclusions. SIAM J. Control Optim. 47(3), 15911614 (2008)

51. Lorenz, T.: Mutational Analysis. Lecture Notes in Mathematics, vol. 1996. Springer, Berlin (2010)

52. Lorenz, T.: Differential Equations for Closed Sets in a Banach Space: Survey and Extension. Vietnam J. Math. (2016). doi:10.1007/s10013-016-0195-x

53. Lorenz, T., Surulescu, C.: On a class of multiscale cancer cell migration models: well-posedness in less regular function spaces. Preprint, TU Kaiserslautern, Department of Mathematics (2013), https:// kluedo.ub.uni-kl.de/frontdoor/index/index/docId/3440

54. Lorenz, T., Surulescu, C.: On a class of multiscale cancer cell migration models: well-posedness in less regular function spaces. Math. Models Methods Appl. Sci. 24(12), 2383-2436 (2014)

55. Maniglia, S.: Probabilistic representation and uniqueness results for measure-valued solutions of transport equations. J. Math. Pure Appl. (9) 87(6), 601-626 (2007)

56. Mucha, P.B.: Transport equation: extension of classical results for div, $b \in$ BMO. J. Differ. Equ. 249(8), 1871-1883 (2010)

57. Hernández Murillo, J.A.: Tangential regularity in the space of directional-morphological transitions. J. Convex Anal. 13(2), 423-441 (2006)

58. Panasyuk, A.I.: Quasidifferential equations in a metric space. Differentsial' nye Uravneniya 21(8), 1344-1353, 1468 (1985)

59. Panasyuk, A.I.: Quasidifferential equations in a complete metric space under Carathéodory-type conditions. I. Differentsial' nye Uravneniya 31(6), 962-972, 1101 (1995)

60. Panasyuk, A.I.: Quasidifferential equations in a complete metric space under Carathéodory-type conditions. II. Differ. Uravn. 31(8), 1361-1369, 1438 (1995)

61. Pavel, N.H.: Nonlinear Evolution Operators and Semigroups. Lecture Notes in Mathematics, vol. 1260. Springer, Berlin (1987)

62. Piccoli, B., Rossi, F.: Transport equation with nonlocal velocity in Wasserstein spaces: convergence of numerical schemes. Acta Appl. Math. 124, 73-105 (2013)

63. Piccoli, B., Rossi, F.: Generalized Wasserstein distance and its application to transport equations with source. Arch. Ration. Mech. Anal. 211(1), 335-358 (2014)

64. Piccoli, B., Tosin, A.: Time-evolving measures and macroscopic modeling of pedestrian flow. Arch. Ration. Mech. Anal. 199(3), 707-738 (2011)

65. Ricceri, B., Villani, A.: Separability and Scorza-Dragoni's property. Matematiche (Catania) 37(1), 156-161 (1985) (1982)

66. Villani, C.: Optimal Transport. Old and New, Grundlehren der mathematischen Wissenschaften [Fundamental Principles of Mathematical Sciences], vol. 338. Springer, Berlin (2009)

67. Walter, W.: Gewöhnliche Differentialgleichungen, 7th edn. Springer-Lehrbuch. [Springer Textbook]. Springer, Berlin (2000). Eine Einführung: An introduction

68. Yosida, K.: Functional analysis, Grundlehren der mathematischen Wissenschaften [Fundamental Principles of Mathematical Sciences], vol. 123, sixth edn. Springer, Berlin (1980)

69. Ziemer, W.P.: Weakly Differentiable Functions. Graduate Texts in Mathematics. Sobolev Spaces and Functions of Bounded Variation, vol. 120. Springer, New York (1989) 